\author{
Universidade de Brasília \\ Instituto de Ciências Exatas \\ Departamento de Matemática
}

\title{
O problema da raiz quadrada de T. Kato para operadores elípticos de segunda ordem em $\mathbb{R}^{n}$
}

\author{
Apresentado POR: \\ Irving Joseph Ramírez Barreto \\ Orientador: Ricardo Parreira da Silva
}




\title{
Irving Joseph Ramírez Barreto
}

\section{O PROBlEMA DA RAIZ QUADRADA DE T. KATO PARA OPERADORES ELÍPTICOS DE SEGUNDA ORDEM EM $\mathbb{R}^{n}$}

\author{
Dissertação apresentada para obtenção do título de \\ Mestre em Matemática, junto ao Programa de \\ Pós-Graduação em Matemática \\ do Instituto de Ciências Exatas, Universidade de \\ Brasília, Campus Darcy Ribeiro.
}

Orientador: Ricardo Parreira DA Silva

FEVEREIRO 2017 


\title{
Irving Joseph Ramírez Barreto
}

\section{O PROBlEMA DA RAIZ QUADRADA DE T. KATO PARA OPERADORES ELÍPTICOS DE SEGUNDA ORDEM EM $\mathbb{R}^{n}$}

\author{
Dissertação apresentada para obtenção do título de \\ Mestre em Matemática, junto ao Programa de \\ Pós-Graduação em Matemática \\ do Instituto de Ciências Exatas, Universidade de \\ Brasília, Campus Darcy Ribeiro.
}

\section{BANCA EXAMINADORA}

\author{
Prof. Ricardo Parreira da Silva \\ $\mathrm{UnB}$ \\ Orientador
}

Prof. Leandro Martins Cioletti $\mathrm{UnB}$

Prof. Jamil Viana Pereira UNESP

Brasília, Distrito Federal

Fevereiro de 2017 


\section{DEDICATÓRIA}

Este trabalho é dedicado especialmente a minha mãe Maria Consuelo e meus irmãos Jaime e Rosa. Apesar da grande distância que nos separa, eles sempre conseguem uma forma de me fazer sentir perto deles. Tenho total certeza que sem ajuda dessa pessoas maravilhosas, não haveria conseguido chegar tão longe na minha vida profissional. Nunca me deixaram cair.

Também dedico este trabalho ao meu tio Victor, quem foi como um pai, e quem infelizmente a vida não deu a oportunidade de me ver crescendo academicamente. Mas, onde seja que você estiver, sei que me esta olhando e dando muitos bom desejos. Neste trabalho coloquei todas as forcas que todos vocês me brindaram .

Não existe amor mais grande do que dá a família. 


\section{Agradecimentos}

Ao longo destes dois anos, é conhecido muita gente que me fez ter uma visão totalmente da vida, me ensinando coisas maravilhosas e vital importância para melhorar como pessoa.

Agradeço principalmente a Deus pela força e proteção que me brindou, por nunca me deixar em tempos difíceis.

Agradeço a minha mãe Maria Consuelo, por ser uma guerreira formidável, por nunca desistir de nossos sonhos e dar tudo de sim para sair adiante como profissionais. Te devo tudo, e sei que minha vida será insuficientemente corta para te compensar por todo o que você me deu.

A meus Irmãos: Jaime e Rosa. Sempre me ajudaram. Mesmo se vocês também tinham suas vidas, seus famílias e seus problemas, tem dedicado um pouco de seu tempo e vontade para me ajudar.

A toda minha família Barreto, pois sempre acreditaram en mim, em que podia conseguir isto.

A meu professor Ricardo Parreira da Silva. Desde o momento em que a gente conversou sobre o trabalho, me deu mais uma esperança de continuar com muita dedicação e empenho. Ele sempre acreditou em mim, em que podia-se terminar o trabalho a tempo. Nunca duvidou da suas palavras e eu acreditei sempre nisso. Obrigado pela amizade e pelo apoio incondicional.

Um agradecimento especial ao professor Pascal Auscher, autor principal do artigo trabalhado. Apesar da pouca informação que eu di para ele, foi muito gentil ao aconselhar algumas dicas e sugestões para o maior entendimento do trabalho.

A Isabel, pela paciência que você sempre tem comigo. Pelo seu carinho e boa vontade de me ajudar de qualquer forma.

A Marisol, por essa amizade única, de quase 9 anos. Você é quem sempre dá o apoio moral, sincero e direto. Mesmo se for difícil de aceitar. Uma verdadeira amizade que vale a pena manter por sempre. 
Agradeço também aos meus amigos peruanos Guillermo e Alan Rafael, e aos amigos peruanos que fiz durante o transcurso do mestrado: Ricardo, Roberto, Jhoel, Rosmery, Josimar, Pedro, Santiago, Juan, Kathy, Cristina e Jamer. Pelos bons momentos que passamos e o apoio que sempre recebemos.

Um agradecimento especial a Roberto, por me ensinar que a família é tudo para nos e que é o motor de nossa vida e sacrifício.

A todos meus amigos brasileiros que eu fiz ao longo deste tempo. São muitas as pessoas que eu conheci e sei que será difícil lembrar de todas elas. Mas agradeço especialmente a Welinton por ser esse 'cara' que me ajudou desde que cheguei, e sempre teve um pouco de tempo para me escutar e me ajudar. Você é verdadeiramente um amigo.

Agradeço à CAPES e CNPq, pelo auxílio financeiro.

E finalmente, porém não menos importante, a Sleyker e a Veldroix.

Não existe amor mais grande, do que dá a família. 


\section{Resumo}

Neste trabalho mostramos a conjetura proposta por Tosio Kato em 1961 para operadores elípticos de segunda ordem na forma divergente em $\mathbb{R}^{n}$. Mais precisamente, estabelecemos que o domínio da raiz quadrada de um operador uniformemente elíptico $L=-\operatorname{div}(A \nabla)$ com coeficientes limitados sobre $\mathbb{R}^{n}$ é o espaço de Sobolev $H^{1}\left(\mathbb{R}^{n}\right)$ com a estimativa $\|\sqrt{L} f\|_{L^{2}} \leq C\|\nabla f\|_{L^{2}}$, para alguma constante $C>0$ que depende apenas de $n$ e das constantes de "elipticidade" de $A$.

Palavras-chave: Operador elíptico, potencias fracionárias, estimativa de medida Carleson, Teorema T(b). 


\section{Abstract}

In this work, we prove the conjecture proposed by Tosio Kato in 1961, for second order elliptic operators in divergence form on $\mathbb{R}^{n}$. More precisely, we establish that the domain of the square root of a uniformly elliptic operator $L=-\operatorname{div}(A \nabla)$ with bounded coefficents in $\mathbb{R}^{n}$ is the Sobolev space $H^{1}\left(\mathbb{R}^{n}\right)$ with the estimate $\|\sqrt{L} f\|_{L^{2}} \leq C\|\nabla f\|_{L^{2}}$, for some constant $C>0$ which depends only of $n$ and the constants of "ellipticity" of $A$.

Key-words: Elliptic operator, fractionary powers, Carleson measure estimate, $\mathrm{T}(\mathrm{b})$ Theorem. 


\section{Sumário}

Introdução 2

1 Preliminares 3

1.1 Formas sesquilineares e operador associado $\ldots \ldots \ldots \ldots$

$1.1 .1 \quad$ Formas sesquilineares limitadas $\ldots \ldots \ldots \ldots \ldots$

1.1.2 Formas sesquilineares ilimitadas . . . . . . . . . . . . 5

1.1.3 Perturbação de formas sesquilineares . . . . . . . . . . . 8

$1.1 .4 \quad$ O Operador Associado . . . . . . . . . . . . . . . 10

1.2 Potências Fracionárias de Operadores $\ldots \ldots \ldots \ldots$

1.2 .1 Potências Fracionárias de Operadores Positivos . . . . . . 13

\begin{tabular}{|lll}
2 & Estimativas de operadores elípticos em $\mathbb{R}^{n}$ & 23
\end{tabular}

$\begin{array}{lll}3 & \text { Redução à estimativa de função quadrática } & 37\end{array}$

3.1 Representação do operador raiz quadrada . . . . . . . . . . . 37

\begin{tabular}{|lll|}
\hline 4 & Redução à estimativa de medida Carleson & 43
\end{tabular}

4.1 Redução à estimativa de função radial $\ldots \ldots \ldots$. . . . . . . . . . 43

4.2 Redução à estimativa de medida Carleson $\ldots \ldots \ldots$. . . . . . 48

\begin{tabular}{|lll}
$5 \quad \mathrm{O}$ argumento $\mathrm{T}(\mathrm{b})$ & 61
\end{tabular}

$5.1 \quad$ O Teorema $\mathrm{T}(\mathrm{b})$ e resultados preliminares $\ldots \ldots \ldots \ldots$. . . . 61

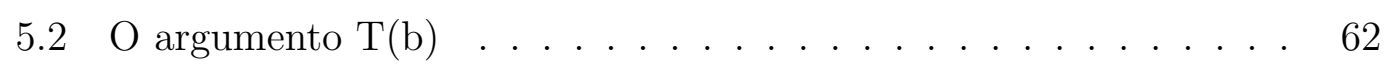

5.3 Conclusão $\ldots \ldots \ldots \ldots \ldots$

A CÁLCULO FUNCIONAL PARA OPERADORES SETORIAIS 81

A.1 Funções holomorfas e o espaço $H_{0}^{\infty} \ldots \ldots \ldots$. . . . . . . . 81

A.2 Cálculo Funcional Limitado . . . . . . . . . . . . . . . . . . . . 83 
B BASE DE RADEMACHER E RESULTADOS AUXILIARES

B.1 Expansões Binárias . . . . . . . . . . . . . . . . . . . . . 85

B.2 Funções de Rademacher . . . . . . . . . . . . . . . . . . . . . . 86

B.3 Estimativas de funções quadráticas $\ldots \ldots \ldots \ldots \ldots$. . . . 87 


\section{Introdução}

O problema da raiz quadrada de Kato foi um problema em aberto por muito tempo. Originalmente proposta por Tosio Kato em 1961 [25], e refinado por Alan $\mathrm{M}^{\mathrm{c}}$ Intosh no contexto de equações de evolução para operadores acretivos maximais sobre espaços de Hilbert, desenvolveu-se até sua formulação atual para operadores diferenciais após a criação de contra-exemplos que foram encontrados para operadores gerais sobre espaços de Hilbert e para certos operadores que surgiram de formas sesquilineares (Veja-se [27] e [28], respectivamente). Porem, foi apontado em [28] que, ao enunciar o problema, Kato tinha sido motivado pelo caso especial de operadores diferenciais elípticos, e pela aplicabilidade de um possível resultado, nesse caso específico, à teoria de perturbação para equações de evolução do tipo parabólicas e hiperbólicas.

O problema da raiz quadrada de Kato, tal como é conhecido atualmente [7], consiste em mostrar que se $A$ é uma matriz de valores complexos $n \times n$, então o operador raiz quadrada, $L^{1 / 2}$, de um operador elíptico de segundo ordem $L=$ $-\operatorname{div}(A \nabla)$ têm domínio $H^{1}$ e satisfaz a estimativa

$$
\|\sqrt{L} f\|_{L^{2}} \sim\|\nabla f\|_{L^{2}}
$$

Esse problema tem uma larga história, e muitas pessoas têm contribuído para a sua solução. Primeiro, Coifman, $\mathrm{M}^{\mathrm{c}}$ Intosh e Meyer provaram a conjectura em uma dimensão, simultaneamente com sua prova da $L^{2}$-limitação da Integral de Cauchy ao longo de uma curva Lipschitz. De fato, ambos resultados são conhecidos por serem equivalentes ( [5] ).

Para dimensões maiores, existem muitos resultados que se aproximaram da solução da conjectura, como Coifman, McIntosh, Meyer, Journé, Semmes, Pascal Auscher e Philippe Tchamitchian([10]), entre outros.

Finalmente, em 2002, junto com Steve Hofmann, Michael Lacey e Alan McIntosh; conseguiram resolver tal conjectura fazendo uso de algumas das seguintes ferramentas: 1) o uso de um cálculo funcional, em particular, estimativas ponto-a- 
ponto do núcleo de um operador integral, 2) a redução a uma estimativa de medida Carleson e 3) a introdução de um "Teorema $\mathrm{T}(\mathrm{b})$ para raízes quadradas "no espírito do teorema T(b) para integrais singulares [33], [30]. Esses T(b) teoremas são todos inspirdos pela integral de Cauchy.

Embora quiséssemos um trabalho totalmente auto-contido, devido ao tempo limitado, teremos que assumir conhecidos alguns elementos de Análise Funcional. Porem tais resultados estao referenciados para uma eventual consulta.

Um detalhe importante a ser mencionado é que a prova em questão é valida para todas dimensões maiores o iguais a 1. Nossa referencia principal para o trabalho foi [7]. 


\section{Capítulo 1}

\section{Preliminares}

\subsection{Formas sesquilineares e operador associado}

\subsubsection{Formas sesquilineares limitadas}

Seja $H$ um espaço de Hilbert sobre $\mathbb{K}=\mathbb{R}$ ou $\mathbb{C}$. Denota-se, nesta seção, por ( ; ) o produto interno de $H$ e por $\|$.$\| sua correspondente norma. Uma forma$ sesquilinear em $H$ é uma aplicação $\mathfrak{a}: H \times H \rightarrow \mathbb{K}$ tal que para cada $\alpha \in \mathbb{K}$ e $u, v \in H:$

$$
\mathfrak{a}(\alpha u+v, h)=\alpha \mathfrak{a}(u, h)+\mathfrak{a}(v, h) \text { e } \mathfrak{a}(u, \alpha v+h)=\bar{\alpha} \mathfrak{a}(u, v)+\mathfrak{a}(u, h),
$$

onde $\bar{\alpha}$ denota o conjugado do número complexo $\alpha$. Claramente, $\bar{\alpha}=\alpha$ se $\mathbb{K}=\mathbb{R}$ e nesse caso a forma a é então bilinear. Por simplicidade não faremos distinção entre os dois casos $\mathbb{K}=\mathbb{R}$ ou $\mathbb{K}=\mathbb{C}$, e usaremos o termo sesquilinear em qualquer das situações.

Definição 1.1 Dizemos que a forma sesquilinear $\mathfrak{a}: H \times H \longrightarrow \mathbb{K}$ é limitada se existe uma constante $M>0$ tal que

$$
|\mathfrak{a}(u, v)| \leq M\|u\|\|v\| \text { para todo } u, v \in H .
$$

Cada forma sesquilinear limitada pode ser representada por um único operador linear limitado. Mais precisamente: 
Proposição 1.2 Assuma que a: $H \times H \longrightarrow \mathbb{K}$ é uma forma sesquilinear limitada. Então existe um único operador linear limitado $T$ atuando em $H$ tal que:

$$
\mathfrak{a}(u, v)=(T u ; v) \text { para todo } u, v \in H
$$

Demonstração: Fixe $u \in H$ e considere o seguinte funcional linear limitado:

$$
\phi(v):=\overline{\mathfrak{a}(u, v)}, v \in H .
$$

Pelo Teorema de Representação de Riesz, existe um único vetor $T u \in H$, tal que

$$
\phi(v)=(v ; T u), \text { para todo } v \in H .
$$

O fato de que $T$ é um operador linear limitado em $H$ segue facilmente das linearidade e limitação da forma a. Para mostrar a unicidade, assuma que $S$ é outro operador satisfazendo a conclusão da proposição. Então

$$
(T u ; v)=(S u ; v), \forall u, v \in H \Longrightarrow(T u-S u ; v)=0, \forall u, v \in H \Longrightarrow T=S . \square
$$

O operador limitado $T$ é o operador associado à forma a . Vamos estudar a invertibilidade de $T$ (ou seu adjunto $T^{*}$ ) usando a forma. Mais precisamente,

Lema 1.3 (Lax-Milgram) Seja a uma forma sesquilinear limitada em $\mathrm{H}$ e assuma que a é coerciva, isto é, existe uma constante $\delta>0$ tal que

$$
\operatorname{Rea}(u, u) \geq \delta\|u\|^{2}, \text { para todo } u \in H
$$

onde Rea denota a parte real de a. Seja $\phi$ um funcional linear limitado em $H$. Então existe um único $v \in H$ tal que:

$$
\phi(u)=\mathfrak{a}(u, v), \text { para todo } u \in H .
$$

Demonstração: Se $T$ denota o operador associado a forma a é suficiente provar que o operador adjunto $T^{*}$ é invertível em $H$. De fato, pelo Teorema de representação de Riesz, existe um único $g \in H$ tal que:

$$
\phi(u)=(u ; g), \text { para todo } u \in H
$$

e então, escrevendo $g=T^{*} v$ para um único $v \in H$, segue que

$$
\phi(u)=\left(u ; T^{*} v\right)=(T u ; v)=\mathfrak{a}(u, v), \text { para todo } u \in H .
$$


Para ver que $T^{*}$ é invertível, seja $v \in H$ tal que $T^{*} v=0$. Portanto,

$$
0=\left(v ; T^{*} v\right)=(T v ; v)=\operatorname{Re} \mathfrak{a}(v, v) \geq \delta\|v\|^{2} .
$$

Logo, $v=0$ e assim, $T^{*}$ é injetiva.

Resta mostrar que $T^{*}$ têm imagem $R\left(T^{*}\right)=H$. Primeiro, vamos provar que $R\left(T^{*}\right)$ é denso. De fato, se $u \in H$ é tal que

$$
\left(u ; T^{*} v\right)=0 \text { para todo } v \in H,
$$

então tomando $v=u$ e usando a condição de coercividade, obtêm-se que $u=$ 0 . Finalmente, vamos mostrar que $R\left(T^{*}\right)$ é fechado. Para isso considere uma sequência $v_{k}=T^{*} u_{k}$ que converge a $v$ em $H$. Assim, segue de:

$$
\begin{aligned}
\delta\left\|u_{k}-u_{j}\right\|^{2} & \leq \operatorname{Re} \mathfrak{a}\left(u_{k}-u_{j}, u_{k}-u_{j}\right) \\
& \leq\left|\left(u_{k}-u_{j} ; T^{*} u_{k}-T^{*} u_{j}\right)\right| \\
& \leq\left\|u_{k}-u_{j}\right\| \cdot\left\|v_{k}-v_{j}\right\|
\end{aligned}
$$

Com isto, concluímos que $\left(u_{k}\right)_{k}$ é uma sequência de Cauchy e portanto, converge em $H$. Se $u$ denota o limite desta convergência, então $v=T^{*} u$ por continuidade de $T^{*}$. Isto mostra que $R\left(T^{*}\right)$ é fechado.

\subsubsection{Formas sesquilineares ilimitadas}

\section{Formas fechadas e fecháveis}

Nesta seção vamos considerar formas sesquilineares que não agem sobre todo o espaço $H$, mas sim em subespaços dele. Essas formas são chamadas formas sesquilineares ilimitadas, as quais têm um papel importante no estudo de equações diferenciais parciais. Chamaremos elas, simplesmente, formas sesquilineares.

Seja $H$ como na seção anterior e seja uma forma sesquilinear a definida sobre um subespaço $D(\mathfrak{a})$ de $H$, chamada domínio de $\mathfrak{a}$. Assim:

$$
\begin{aligned}
\mathfrak{a}: D(\mathfrak{a}) \times D(\mathfrak{a}) & \longrightarrow \mathbb{K} \\
(u, v) & \longrightarrow \mathfrak{a}(u, v)
\end{aligned}
$$

satisfazendo a condição 1.1 de forma sesquilinear. 
Definição 1.4 Seja $\mathfrak{a}: D(\mathfrak{a}) \times D(\mathfrak{a}) \longrightarrow \mathbb{K}$ uma forma sesquilinear. Dizemos que:

1) a é densamente definida se:

$$
D(\mathfrak{a}) \text { é denso em } H \text {. }
$$

2) a é acretiva se:

$$
R e \mathfrak{a}(u, u) \geq 0, \forall u \in D(\mathfrak{a}) .
$$

3) a é contínua se existe uma constante positiva $M$ tal que:

$$
|\mathfrak{a}(u, v)| \leq M\|u\|_{\mathfrak{a}}\|v\|_{\mathfrak{a}}, \forall u, v \in D(\mathfrak{a})
$$

onde $\|u\|_{\mathfrak{a}}:=\sqrt{\mathfrak{a}(u, u)+\|u\|^{2}}$

4) a é fechada se:

$$
\left(D(\mathfrak{a}),\|\|_{\mathfrak{a}}\right) \text { é um espaço de Banach. }
$$

Se $\mathfrak{a}$ satisfaz (1.2)-(1.5), pode-se mostrar facilmente que \|\|$_{\mathfrak{a}}$ é uma norma sobre $D(\mathfrak{a})$. Esta norma é chamada norma associada à forma $\mathfrak{a}$.

Definição 1.5 Seja a uma forma sesquilinear sobre $H$. A forma adjunta de a é a forma sesquilinear $\mathfrak{a}^{*}$ definida por:

$$
\mathfrak{a}^{*}(u, v):=\overline{\mathfrak{a}(v, u)}, \forall u, v \in D(\mathfrak{a}), \operatorname{com} D\left(\mathfrak{a}^{*}\right)=D(\mathfrak{a})
$$

A parte simétrica de $\mathfrak{a}$ é definida por $\mathfrak{b}:=\frac{1}{2}\left(\mathfrak{a}+\mathfrak{a}^{*}\right)$, onde $D(\mathfrak{b})=D(\mathfrak{a})$. Dizemos que $\mathfrak{a}$ é simétrica se $\mathfrak{a}^{*}=\mathfrak{a}$, i.e., $\mathfrak{a}(u, v)=\overline{\mathfrak{a}(v, u)}, \forall u, v \in D(\mathfrak{a})$.

Se a é uma forma sesquilinear que satisfaz (1.2)-(1.5), então $D(\mathfrak{a})$ é um espaço de Hilbert. Neste caso, o produto interno é definido por:

$$
(u ; v)_{\mathfrak{a}}:=\frac{1}{2}\left[\mathfrak{a}(u, v)+\mathfrak{a}^{*}(u, v)\right]+(u ; v) \text { para todo } u, v \in D(\mathfrak{a}) .
$$

Ao longo desta seção, vamos considerar somente formas acretivas (i.e., que satisfazem (1.3)). Poderíamos, em vez disso, considerar formas que são meramente limitadas inferiormente, isto é:

$$
R e \mathfrak{a}(u ; u) \geq-\gamma(u ; u), \forall u \in D(\mathfrak{a})
$$

para alguma constante positiva $\gamma$. 
A teoria geral dessas formas não têm muita diferença daquela para formas acretivas. Uma simples perturbação (a qual consiste em considerar a forma $\mathfrak{a}+\gamma$, definida por $(\mathfrak{a}+\gamma)(u, v):=\mathfrak{a}(u, v)+\gamma(u, v), \forall u, v \in D(\mathfrak{a}))$ nos permite considerar somente formas acretivas.

Se a é uma forma simétrica, a propriedade de acretividade (1.3) nos diz que $\mathfrak{a}$ é não negativa, ou seja:

$$
\mathfrak{a}(u, u) \geq 0, \forall u \in D(\mathfrak{a})
$$

Assim, para formas simétricas, vamos usar os termos 'acretivo' e 'positivo' para nos referir à propriedade (1.3).

Proposição 1.6 Seja $\mathfrak{a}: H \times H \longrightarrow \mathbb{K}$ uma forma sesquilinear acretiva $e$ fechada. Então as normas:

$$
\|\cdot\| e\|\cdot\|_{\mathfrak{a}}
$$

são equivalentes em $H$.

Prova: Para cada $u \in H$, temos:

$$
\|u\| \leq\left(\|u\|^{2}\right)^{\frac{1}{2}} \leq \sqrt{\left(\|u\|^{2}+\operatorname{Re} \mathfrak{a}(u, u)\right)}=\|u\|_{\mathfrak{a}} .
$$

Em outras palavras, o operador $I:\left(H,\|\|_{\mathfrak{a}}\right) \longrightarrow(H,\|\|)$ é contínuo. Do Teorema da aplicação aberta segue que as duas normas são equivalentes.

Uma condição mais forte que a continuidade é a "setorialidade", a qual vemos na seguinte definição.

Definição 1.7 A forma sesquilinear $\mathfrak{a}: D(\mathfrak{a}) \times D(\mathfrak{a}) \longrightarrow \mathbb{C}$, agindo sobre um espaço de Hilbert complexo é chamada setorial se existe uma constante não negativa $C$, tal que:

$$
|\operatorname{Im} \mathfrak{a}(u, u)| \leq C|\operatorname{Re} \mathfrak{a}(u, u)|, \forall u \in D(\mathfrak{a})
$$

A imagem numérica de a é o conjunto:

$$
\mathcal{N}(\mathfrak{a}):=\{\mathfrak{a}(u, u), u \in D(\mathfrak{a}), \operatorname{com}\|u\|=1\}
$$

Claramente, $\mathfrak{a}$ satisfaz (1.6) se e somente se a imagem numérica $\mathcal{N}(\mathfrak{a})$ está contida no setor fechado do plano complexo $\{z \in \mathbb{C}:|\arg z| \leq \operatorname{arctg} C\}$. 
Proposição 1.8 Toda forma setorial atuando sobre um espaço de Hilbert complexo $H$ é contínua. Mais exatamente, se existe $C>0$ tal que

$$
|\operatorname{Im} \mathfrak{a}(u, u)| \leq C R e \mathfrak{a}(u, u), \forall u \in D(\mathfrak{a})
$$

então

$$
|\mathfrak{a}(u, v)| \leq(1+C)(\operatorname{Re} \mathfrak{a}(u, u))^{1 / 2} \cdot(\operatorname{Re} \mathfrak{a}(v, v))^{1 / 2}, \forall u \in D(\mathfrak{a})
$$

Demonstração: Veja [32]

A recíproca da proposição 1.8 é dada pelo seguinte lema.

Lema 1.9 Se a é uma forma sesquilinear acretiva e contínua sobre um espaço de Hilbert complexo $H$, então a forma:

$$
(;)+\mathfrak{a}
$$

é setorial. Mais exatamente, se a satisfaz (1.4) para alguma constante $M$, então:

$$
\operatorname{Im}[(u ; u)+\mathfrak{a}(u, u)] \leq M \operatorname{Re}[(u ; u)+\mathfrak{a}(u, u)], \forall u \in D(\mathfrak{a})
$$

Prova: Observa-se facilmente que, como $(u ; u) \in \mathbb{R}$ :

$$
|\operatorname{Im}[(u ; u)+\mathfrak{a}(u, u)]|=|\operatorname{Im} \mathfrak{a}(u, u)| \leq|\mathfrak{a}(u, u)|
$$

e usando a condição (1.4), tem-se que:

$$
\begin{aligned}
\operatorname{Im}[(u ; u)+\mathfrak{a}(u, u)] & \leq M\|u\|_{\mathfrak{a}}\|u\|_{\mathfrak{a}}=M\|u\|_{\mathfrak{a}}^{2}=M\left(\|u\|^{2}+\operatorname{Re} \mathfrak{a}(u, u)\right) \\
& =M \operatorname{Re}[(u ; u)+\mathfrak{a}(\mathfrak{u}, \mathfrak{u})], \quad \forall u \in D(\mathfrak{a}) .
\end{aligned}
$$

\subsubsection{Perturbação de formas sesquilineares}

Iniciamos esta seção definindo a soma $\mathfrak{a}+\mathfrak{b}$ de duas formas $\mathfrak{a}$ e $\mathfrak{b}$ sobre $H$, como:

$$
[\mathfrak{a}+\mathfrak{b}](u, v):=\mathfrak{a}(u, v)+\mathfrak{b}(u, v), \operatorname{com} D(\mathfrak{a}+\mathfrak{b}):=D(\mathfrak{a}) \cap D(\mathfrak{b})
$$

e a norma associada a essa forma é dada por:

$$
\|u\|_{\mathfrak{a}+\mathfrak{b}}:=\sqrt{\operatorname{Re}[\mathfrak{a}+\mathfrak{b}](u, u)+\|u\|^{2}}, \forall u \in D(\mathfrak{a}+\mathfrak{b})=D(\mathfrak{a}) \cap D(\mathfrak{b})
$$


Teorema 1.10 Seja $\mathfrak{a}$ e $\mathfrak{b}$ duas formas sesquilineares acretivas sobre $H$. Então a soma $\mathfrak{a}+\mathfrak{b}$ é acretiva. Mais ainda:

(i) Se $\mathfrak{a} e \mathfrak{b}$ são contínuas, então $\mathfrak{a}+\mathfrak{b}$ também o é.

(ii) Se $\mathfrak{a}$ e $\mathfrak{b}$ são fechadas, então $\mathfrak{a}+\mathfrak{b}$ também o é.

Demonstração: A acretividade sai diretamente da definição da soma de formas sesquilineares. Para mostrar a continuidade, observe que:

$$
\begin{aligned}
|[\mathfrak{a}+\mathfrak{b}](u, v)| & \leq|\mathfrak{a}(u, v)+\mathfrak{b}(u, v)| \leq|\mathfrak{a}(u, v)|+|\mathfrak{b}(u, v)| \\
& \leq M_{1}\left(\|u\|_{\mathfrak{a}}\|v\|_{\mathfrak{a}}\right)+M_{2}\left(\|u\|_{\mathfrak{b}}\|v\|_{\mathfrak{b}}\right)
\end{aligned}
$$

Já que $\|u\|_{\mathfrak{a}+\mathfrak{b}} \geq\|u\|_{\mathfrak{a}}$ e $\|u\|_{\mathfrak{a}+\mathfrak{b}} \geq\|u\|_{\mathfrak{b}}, \forall u \in D(\mathfrak{a}+\mathfrak{b})$, da desigualdade anterior segue que:

$$
\begin{aligned}
{[\mathfrak{a}+\mathfrak{b}](u, v) \mid } & \leq M_{1}\left(\|u\|_{\mathfrak{a}}\|v\|_{\mathfrak{a}}\right)+M_{2}\left(\|u\|_{\mathfrak{b}}\|v\|_{\mathfrak{b}}\right) \\
& \leq\left(M_{1}+M_{2}\right)\|u\|_{\mathfrak{a}+\mathfrak{b}}\|v\|_{\mathfrak{a}+\mathfrak{b}} \\
& =M\|u\|_{\mathfrak{a}+\mathfrak{b}}\|v\|_{\mathfrak{a}+\mathfrak{b}} .
\end{aligned}
$$

Agora suponha que $\mathfrak{a}$ e $\mathfrak{b}$ são fechados. Seja $\left\{u_{n}\right\}_{n}$ uma sequência de Cauchy em $D(\mathfrak{a}+\mathfrak{b})$ com sua respetiva norma. Observamos que:

$$
\left\|u_{n}-u_{m}\right\|_{\mathfrak{a}} \leq\left\|u_{n}-u_{m}\right\|_{\mathfrak{a}+\mathfrak{b}} \longrightarrow 0 \text { e }\left\|u_{n}-u_{m}\right\|_{\mathfrak{b}} \leq\left\|u_{n}-u_{m}\right\|_{\mathfrak{a}+\mathfrak{b}} \longrightarrow 0
$$

quando $m, n \longrightarrow \infty$. Logo $\left\{u_{n}\right\}_{n}$ é uma sequência de Cauchy com as normas \|\|$_{\mathfrak{a}}$ e \|\|$_{\mathfrak{b}}$. Segue então, por hipótese, que $\left\{u_{n}\right\}_{n}$ converge nos espaços $\left(D(\mathfrak{a}),\|\|_{\mathfrak{a}}\right)$ e $\left(D(\mathfrak{b}),\|\|_{\mathfrak{b}}\right)$. O limite nesses espaços é o mesmo, já que a convergência em cada um deles implica a convergência no espaço $H$. Portanto, o limite pertence ao espaço $D(\mathfrak{a}+\mathfrak{b})$.

Resta provar que $\left\{u_{n}\right\}_{n}$ converge na norma \|\|$_{\mathfrak{a}+\mathfrak{b}}$. Para isso, note que:

$$
\begin{aligned}
\|u\|_{\mathfrak{a}+\mathfrak{b}} & =\sqrt{\operatorname{Re}[\mathfrak{a}+\mathfrak{b}](u, u)+\|u\|_{H}^{2}} \\
& \leq \sqrt{\operatorname{Re} \mathfrak{a}(u, u)+\operatorname{Re} \mathfrak{b}(u, u)+\|u\|_{H}^{2}+\|u\|_{H}^{2}} \\
& =\sqrt{\|u\|_{\mathfrak{a}}^{2}+\|u\|_{\mathfrak{b}}^{2}} \\
& \leq\|u\|_{\mathfrak{a}}+\|u\|_{\mathfrak{b}} .
\end{aligned}
$$

Logo $\left\|u_{n}-u\right\|_{\mathfrak{a}+\mathfrak{b}} \leq\left\|u_{n}-u\right\|_{\mathfrak{a}}+\left\|u_{n}-u\right\|_{\mathfrak{b}} \longrightarrow 0$, quando $m, n \longrightarrow \infty$, mostrando que $\mathfrak{a}+\mathfrak{b}$ é fechada. 


\subsubsection{O Operador Associado}

Considere uma forma sesquilinear a contínua, fechada, acretiva e densamente definida sobre $H$. Pode-se definir em termos de $\mathfrak{a}$ um operador ilimitado $A$, definido sobre um subespaço $D(A)$ de $H$, como sendo:

$u \in D(\mathfrak{a})$ está no domínio de $A, D(A)$ se, e somente se, existe $v \in H$ tal que a igualdade:

$$
\mathfrak{a}(u, \phi)=(v ; \phi)
$$

vale para todo $\phi \in D(\mathfrak{a})$, e $A u:=v$.

Reescrevemos isto como:

$$
D(A)=\{u \in H \quad: \exists v \in H \operatorname{com} \mathfrak{a}(u, \phi)=(v ; \phi) \forall \phi \in D(\mathfrak{a})\}, A u:=v .
$$

Observação: $D(A)$ é o conjunto de vetores $u \in D(\mathfrak{a})$ tal que a aplicação $\phi \longrightarrow$ $\mathfrak{a}(u, \phi)$ é contínua em $D(\mathfrak{a})$ com respeito à norma de $H$.

Definição 1.11 O operador ilimitado A, definido anteriormente, é chamado operador associado à forma $\mathfrak{a}$.

Existem várias propriedades importantes de operadores associados com formas sesquilineares. Começamos com o seguinte resultado que será usado nos capítulos seguintes:

Teorema 1.12 Seja a uma forma sesquilinear contínua, acretiva, fechada e densamente definida; e A o seu operador associado. Então A é densamente definido e para cada $\lambda>0$, operador $\lambda I+A$ é invertível (de $D(A)$ em $H$ ) e sua inversa $(\lambda I+A)^{-1}$ é um operador limitado sobre $H$ (onde $I$ é o operador identidade de H). Mais ainda:

$$
\left\|\lambda(\lambda I+A)^{-1} f\right\| \leq\|f\|, \forall \lambda>0, \forall f \in H .
$$

Demonstração: Fixa-se $\lambda>0$ e considere:

$$
\|u\|_{\lambda}:=\sqrt{\operatorname{Re} \mathfrak{a}(u, u)+\lambda\|u\|^{2}}, \quad u \in D(\mathfrak{a}) .
$$

Afirmamos que \|\|$_{\lambda}$ e \|\|$_{\mathfrak{a}}$ são normas equivalentes. De fato, dado $\lambda>1$ $\left(\lambda^{-1}<1\right)$, segue que:

$$
\begin{aligned}
\frac{1}{\lambda^{2}}\|u\|_{\lambda} & =\sqrt{\frac{1}{\lambda} \operatorname{Re} \mathfrak{a}(u, u)+\frac{\lambda}{\lambda}\|u\|^{2}} \leq \sqrt{\operatorname{Re} \mathfrak{a}(u, u)+\|u\|^{2}} \\
& =\|u\|_{\mathfrak{a}} \\
& \leq \sqrt{\operatorname{Re} \mathfrak{a}(u, u)+\lambda\|u\|^{2}} \leq\|u\|_{\lambda}
\end{aligned}
$$


Por tanto, $\lambda^{-2}\|u\|_{\lambda} \leq\|u\|_{\mathfrak{a}} \leq\|u\|_{\lambda}$. Analogamente, para $\lambda<1$, tem-se:

$$
\|u\|_{\lambda} \leq\|u\|_{\mathfrak{a}} \leq \lambda^{-2}\|u\|_{\lambda}
$$

Logo, as duas normas são equivalentes. Com isso, o espaço $V:=\left(D(\mathfrak{a}),\|\cdot\|_{\lambda}\right)$ é um espaço de Hilbert. Por outro lado, sabe-se que $\|u\| \leq\|u\|_{\mathfrak{a}} \leq M\|u\|_{\lambda}$. Assim por (1.4) e Definição 1.5, a forma $\lambda+\mathfrak{a}^{*}$ definida como:

$$
\left(\lambda+\mathfrak{a}^{*}\right)(u, v)=\lambda(u ; v)+\mathfrak{a}^{*}(u, v)=\lambda(u ; v)+\overline{\mathfrak{a}(v, u)}
$$

é uma forma limitada sobre $V$. De fato:

$$
\begin{aligned}
\left|\left(\lambda+\mathfrak{a}^{*}\right)(u, v)\right| & =|\lambda(u ; v)+\overline{\mathfrak{a}(v, u)}| \\
& \leq \lambda\|u\|\|v\|+|\overline{\mathfrak{a}(v, u)}|=\lambda\|u\|\|v\|+|\mathfrak{a}(v, u)| \\
& \leq \lambda\|u\|_{\mathfrak{a}}\|v\|_{\mathfrak{a}}+\bar{M}\|u\|_{\mathfrak{a}}\|v\|_{\mathfrak{a}} \\
& \leq C\|u\|_{\mathfrak{a}}\|v\|_{\mathfrak{a}} \\
& \leq M\|u\|_{\lambda}\|v\|_{\lambda} .
\end{aligned}
$$

Além disso:

$$
\begin{aligned}
\operatorname{Re}\left(\lambda+\mathfrak{a}^{*}\right)(u, u) & =\operatorname{Re} \lambda(u ; u)+\operatorname{Re} \overline{\mathfrak{a}(u, u)} \\
& =\lambda(u ; u)+\operatorname{Re} \mathfrak{a}(u, u) \\
& =\lambda\|u\|^{2}+\operatorname{Re} \mathfrak{a}(u, u)=\|u\|_{\lambda}^{2}, \forall u \in V .
\end{aligned}
$$

Logo, $\lambda+\mathfrak{a}^{*}$ é coerciva. Seja $f \in H$ e defina o funcional linear:

$$
\phi(v):=(v ; f), v \in V
$$

Usando o fato que $\|u\| \leq M\|u\|_{\lambda}$, temos :

$$
|\phi(v)| \leq\|v\| \cdot\|f\| \leq M\|v\|_{\lambda} \cdot\|f\|=M\|v\|_{\lambda}
$$

i.e, a função $\phi$ é uma função contínua sobre $V$. Segue do Lema 1.3 (Lax-Milgram) que existe um único $u \in V$ tal que:

$$
\phi(v)=\left(\lambda+\mathfrak{a}^{*}\right)(v, u)=\mathfrak{a}^{*}(v, u)+\lambda(v ; u)=\overline{\mathfrak{a}(u, v)}+\lambda(v ; u), \forall v \in V
$$

Segue então que $(v ; f)=\overline{\mathfrak{a}(u, v)}+\lambda(v ; u)=\overline{(\lambda+\mathfrak{a})(v, u)}$. Com este resultado, e a definição de $A$, segue que $u \in D(A)$ e $(\lambda I+A) u=f$. Pela escolha arbitrária 
de $f$, o operador $\lambda I+A$ têm imagem $R(\lambda I+A)=H$, i.e. $\lambda I+A$ é sobrejetiva. Da condição de acretividade, se $(\lambda I+A) u=0$, então:

$$
\begin{aligned}
0 & =\lambda(u ; u)+\mathfrak{a}(u, u) \\
& =\lambda(u ; u)+\operatorname{Re} \mathfrak{a}(u, u) \\
& \geq \lambda(u ; u)=\lambda\|u\|^{2}
\end{aligned}
$$

Isso implica que $u=0$ e portanto, $\lambda I+A$ é injetiva. Concluímos que $\lambda I+A$ é invertível.

Agora, tomamos $f \in H$ e $u \in D(A)$ de maneira que $(\lambda I+A) u=f$, temos

$$
\begin{aligned}
\|f\|\|u\| & \geq|(f ; u)| \geq \operatorname{Re}(f ; u) \\
& =\operatorname{Re}(\lambda(u ; u)+(A u ; u)) \\
& =\operatorname{Re}(\lambda(u ; u)+\mathfrak{a}(u, u)) \\
& =\lambda(u ; u)+\operatorname{Re} \mathfrak{a}(u, u) \\
& \geq \lambda\|u\|^{2}
\end{aligned}
$$

Logo, $\lambda\|u\| \leq\|f\|$, ou seja, $\lambda\left\|(\lambda I+A)^{1} f\right\| \leq\|f\|$, e como $\lambda$ é positivo, temos finalmente que:

$$
\left\|\lambda(\lambda I+A)^{-1} f\right\| \leq\|f\| .
$$

Tomando-se supremo com respeito a $f$, segue que $(\lambda I+A)^{-1}$ é um operador limitado sobre $H$. Para finalizar a demonstração, resta provar que $D(A)$ é denso em $H$. Considere $v \in H$ de modo que:

$$
(v ; u)=0, \forall u \in D(A)
$$

Como $I+A$ é invertível, existe $\psi \in D(A)$, tal que: $(I+A) \phi=v$. Usando a desigualdade anterior com $u=\phi$, tem-se:

$$
0=(v ; \phi)=((I+A) \phi ; \phi)=\|\phi\|^{2}+(A \phi ; \phi) \geq\|\phi\|^{2}+R e(A \phi ; \phi) \geq\|\phi\|^{2}
$$

$\Rightarrow \phi=0, \log 0 v=0$. Assim, concluímos que $D(A)$ é denso em $H$.

Corolário 1.13 O operador $\left(I+t^{2} A\right)^{-1}$, definido sobre $D(A)$, é uniformemente limitado em $H$, para qualquer $t>0$.

Prova: Do teorema anterior, considerando $\lambda=t^{-2}$, segue que $\left(t^{-2} I+A\right)^{-1}$ é um operador limitado em $H$. Alem disso, a estimativa:

$$
\left(I+t^{2} A\right)^{-1}=\left\|t^{-2}\left(t^{-2} I+A\right)^{-1} f\right\| \leq\|f\|
$$

é satisfeita para qualquer $f \in H$, e portanto

$$
\left\|\left(I+t^{2} A\right)^{-1}\right\| \leq 1
$$




\subsection{Potências Fracionárias de Operadores}

Seja $X$ um espaço de Banach sobre $\mathbb{K}=\mathbb{C}$ ou $\mathbb{R}$, e $A: D(A) \subset X \longrightarrow X$ um operador linear fechado.

Definição 1.14 O conjunto resolvente de A, é definido por:

$$
\begin{aligned}
\rho(A):= & \{\lambda \in \mathbb{C}: \lambda I-A: D(A) \rightarrow X \text { é injetiva, sobrejetiva } e \\
& \left.(\lambda I-A)^{-1}: X \longrightarrow X \text { é limitada. }\right\}
\end{aligned}
$$

Para $\lambda \in \rho(A)$, o operador $(\lambda I-A)^{-1}$ é chamado operador resolvente e o denotamos também por $R(\lambda: A)$.

Definição $1.15 O$ espectro de $A$ é o conjunto $\sigma(A):=\mathbb{C} \backslash \rho(A)$.

\subsubsection{Potências Fracionárias de Operadores Positivos}

Definição 1.16 Um operador $A: D(A) \subset X \longrightarrow X$ é chamado um operador positivo ( de tipo $N \geq 1$ ) se:

(i) A é um operador fechado densamente definido.

(ii) $(-\infty, 0] \subset \rho(A)$

(iii) $\exists N \geq 1, \forall s \leq 0\|R(s: A)\| \leq \frac{N}{1+|s|}$

Lema 1.17 Se $A: D(A) \subset X \longrightarrow X$ é um operador positivo de tipo $N \geq 1$, então:

(i) A é um operador fechado densamente definido,

(ii) $\Sigma_{N}=\left\{\lambda \in \mathbb{C}: \exists s \leq 0,|\lambda-s| \leq \frac{1+|s|}{2 N}\right\} \subset \rho(A)$,

(iii) $\forall \lambda \in \Sigma_{N}$ temos que: $\|R(\lambda: A)\| \leq \frac{2 N+1}{1+|\lambda|}$

Demonstração: Fixe $\lambda \in \Sigma_{N}$. Então existe um $s \geq 0$ tal que $|\lambda-s| \leq \frac{1+|s|}{2 N}$. Além disso, temos a seguinte identidade:

$$
\lambda I-A=(s I-A)(I+(\lambda-s) R(s: A))
$$

Tomando $B=I+(\lambda-s) R(s: A) \in \mathcal{L}(X)$, pode-se observar que :

$$
\|I-B\|=|\lambda-s|\|R(s: A)\| \leq \frac{1+|s|}{2 N} \frac{N}{1+|s|}=\frac{1}{2}<1
$$


Segue que existe $B^{-1} \in \mathcal{L}(X)$ e $\left\|B^{-1}-I\right\| \leq \frac{\|I-B\|}{1-\|I-B\|} \leq 1$. Portanto $\lambda \in \rho(A)$ e, da inversa da identidade anterior, $R(\lambda: A)=B^{-1} R(s: A)$. Mais ainda:

$$
\begin{aligned}
\|R(\lambda: A)\| & =\left\|\left(B^{-1}-I\right) R(s: A)+R(s: A)\right\| \\
& \leq\left\|B^{-1}-I\right\|\|R(s: A)\|+\|R(s: A)\| \\
& \leq 2\|R(s: A)\| \\
& \leq \frac{2 N}{1+|s|}=\frac{2 N}{1+|s|} \cdot \frac{1+|\lambda+s-s|}{1-|\lambda|} \\
& \leq \frac{2 N}{1+|\lambda|} \cdot \frac{1+|s|+|\lambda-s|}{1+|s|} \\
& \leq \frac{2 N}{1+|\lambda|}\left(1+\frac{1}{2 N}\right) \\
& =\frac{2 N+1}{1+|\lambda|}
\end{aligned}
$$

completando a prova. $\square$

Observação: Note que:

$$
\Omega=\left\{\lambda \in \mathbb{C}:|\arg \lambda| \geq \pi-\arcsin \frac{1}{2 N}\right\} \cup\left\{\lambda \in \mathbb{C}:|\lambda| \leq \frac{1}{2 N}\right\}
$$

está contido em $\Sigma_{N}$.

Proposição 1.18 Seja $A: D(A) \subset X \longrightarrow X$ um operador positivo de tipo $N \geq 1$. Para $z \in \mathbb{C}$ tal que Re $z<0$ definimos:

$$
B(z):=-\frac{1}{2 \pi i} \int_{\Gamma} \lambda^{z} R(\lambda: A) d \lambda
$$

onde $\Gamma$ é a curva em $\Sigma_{N} \backslash(-\infty, 0]$, consistindo de três segmentos

$$
\left\{-s e^{-i \theta}: s \in\left(-\infty,-\frac{1}{4 N}\right]\right\},\left\{\frac{1}{4 N} e^{i \psi}:|\psi| \leq \theta\right\},\left\{s e^{i \theta}: s \in\left(\frac{1}{4 N}, \infty\right]\right\}
$$

$\operatorname{com} \theta \in\left[\pi-\arcsin \frac{1}{2 N}, \pi\right)$, que são orientados por suas parametrizações. Então $B(z) \in \mathcal{L}(X)$ está bem definido para $z \in \mathbb{C}$ tal que Re $z<0$, e, para um z fixo a integral não depende da escolha de $\theta$. Além disso, a aplicação $z \longrightarrow B(z)$ é analítica em $\{z \in \mathbb{C}:$ Re $z<0\}$.

Observação: Destacamos que o comportamento da curva $\Gamma$ em (1.9) em torno de 0 pode ser modificada de acordo com o Teorema de Cauchy.

Agora somos capazes de definir as potências fracionárias de um operador positivo para $z \in \mathbb{C}$ com $\operatorname{Re} z<0$. 
Definição 1.19 Seja $A: D(A) \subset X \longrightarrow X$ um operador positivo de tipo $N \geq 1$. Para $z \in \mathbb{C}$ com Re $z<0$ definimos:

$$
A^{z}:=-\frac{1}{2 \pi i} \int_{\Gamma} \lambda^{z} R(\lambda: A) d \lambda \in \mathcal{L}(X)
$$

onde $\Gamma$ é dado por (1.9) para qualquer $\theta \in\left[\pi-\arcsin \frac{1}{2 N}, \pi\right)$. Além disso, temos:

$$
A^{0}=I
$$

onde $I$ denota o operador identidade sobre $X$.

A seguir, mostraremos que o produto de potências fracionárias de um operador com exponentes complexos com parte real negativa é também uma potência cujo exponente é a soma dos exponentes.

Lema 1.20 Seja $A: D(A) \subset X \longrightarrow X$ um operador positivo. Então temos que:

$$
A^{z_{1}+z_{2}}=A^{z_{1}} A^{z_{2}}, z_{1}, z_{2} \in\{z \in \mathbb{C}: \operatorname{Re} z<0\} \cup\{0\}
$$

Demonstração: Tome $A: D(A) \subset X \longrightarrow X$ um operador positivo de tipo $N \geq 1$. Se $z_{1}$ ou $z_{2}$ são zero, então a afirmação é obvia. Fixa-se $z_{1}, z_{2} \in \mathbb{C}$ tal que $R e z_{1}<0$, Re $z_{2}<0$ e considere as curvas $\Gamma_{1}$ e $\Gamma_{2}$ de tal maneira que $\Gamma_{1}$ esteja à direita de $\Gamma_{2} \mathrm{e}$

$$
A^{z_{1}}=-\frac{1}{2 \pi i} \int_{\Gamma_{1}} \lambda^{z_{1}} R(\lambda: A) d \lambda, A^{z_{2}}=-\frac{1}{2 \pi i} \int_{\Gamma_{2}} \mu^{z_{2}} R(\mu: A) d \mu
$$

É possível escolher tal $\Gamma_{1}$ e $\Gamma_{2}$ deixando a $\Gamma_{1}$ ser a curva $\Gamma$ como em (1.9) para algum $\theta_{1}$ e $\Gamma_{2}$ sendo a mesma curva $\Gamma$ com algum $\theta_{2}>\theta_{1}$ e adequadamente modificada em torno de 0 de acordo com o Teorema de Cauchy. Então temos para $x \in X$ :

$$
\begin{aligned}
A^{z_{1}} A^{z_{2}} x & =\frac{1}{(2 \pi i)^{2}} \int_{\Gamma_{1}} \lambda^{z_{1}} R(\lambda: A) \int_{\Gamma_{2}} \mu^{z_{2}} R(\mu: A) x d \mu d \lambda \\
& =\frac{1}{(2 \pi i)^{2}} \int_{\Gamma_{2}} \int_{\Gamma_{1}} \lambda^{z_{1}} \mu^{z_{2}} R(\lambda: A) R(\mu: A) x d \lambda d \mu \\
& =\frac{1}{(2 \pi i)^{2}}\left[\iint_{\Gamma_{2}} \frac{\lambda^{z_{1}} \mu^{z_{2}}}{\mu-\lambda} R(\lambda: A) x d \lambda d \mu-\int_{\Gamma_{2}} \mu^{z_{2}}\left(\int_{\Gamma_{1}} \frac{\lambda^{z_{1}}}{\mu-\lambda} R(\mu: A) x d \lambda\right) d \mu\right]
\end{aligned}
$$

Aqui, usamos o teorema de Fubini e a seguinte formula do resolvente:

$$
R(\lambda: A)-R(\mu: A)=(\mu-\lambda) R(\lambda: A) R(\mu: A)
$$


para as anteriores identidades. Ao mesmo tempo, afirmamos que para um $\mu \in \Gamma_{2}$ fixo, temos:

$$
\int_{\Gamma_{1}} \frac{\lambda^{z_{1}}}{\mu-\lambda} R(\mu: A) x d \lambda=0
$$

De fato, fechamos a curva $\Gamma_{1}$ por meio dos $\operatorname{arcos} C_{s}=\left\{s e^{i \psi}:-\theta_{1} \leq \psi \leq \theta_{1}\right\}$ com $s$ positivo e suficientemente grande. Então para tal $s$ tem-se:

$$
\begin{aligned}
\left\|\int_{C_{s}} \frac{\lambda^{z_{1}}}{\mu-\lambda} R(\mu: A) x d \lambda\right\| & \leq\|R(\mu: A) x\| \int_{-\theta_{1}}^{\theta_{2}} \frac{e^{R e z_{1} \ln s-\psi \operatorname{Im} z_{1}}}{\mid \mu-s e^{i \psi \mid}} s d \psi \\
& \leq s^{R e} z_{1} \frac{s}{s-|\mu|}\|R(\mu: A) x\| \int_{-\theta_{1}}^{\theta_{1}} e^{-\psi \operatorname{Im} z_{1}} d \psi \\
& \longrightarrow 0, \text { quando } s \rightarrow \infty .
\end{aligned}
$$

Já que $\Gamma_{1}$ está à direita de $\Gamma_{2}$ o teorema de Cauchy garante (1.9).Então temos:

$$
A^{z_{1}} A^{z_{2}} x=\frac{1}{(2 \pi i)^{2}} \int_{\Gamma_{2}} \int_{\Gamma_{1}} \frac{\lambda^{z_{1}} \mu^{z_{2}}}{\mu-\lambda} R(\lambda: A) x d \lambda d \mu
$$

Aplicando novamente o teorema de Fubini, deduzimos que:

$$
A^{z_{1}} A^{z_{2}} x=\frac{1}{(2 \pi i)^{2}} \int_{\Gamma_{1}} \lambda^{z_{1}}\left(\int_{\Gamma_{2}} \frac{\mu^{z_{2}}}{\mu-\lambda} R(\lambda: A) x d \mu\right) d \lambda
$$

A fim de calcular a integral interior de (1.12), fechamos $\Gamma_{2}$ por meio de arcos $C_{s}^{\prime}=\left\{s e^{i \psi}:-\theta_{2} \leq \psi \leq \theta_{2}\right\}$ para um $s$ positivo e suficientemente grande. Então para tal $s$, observa-se que:

$$
\left\|\int_{C_{s}^{\prime}} \frac{\mu^{z_{2}}}{\mu-\lambda} R(\lambda: A) x d \mu\right\| \leq s^{R e} z_{2} \frac{s}{s-|\lambda|}\|R(\lambda: A) x\| \int_{-\theta_{2}}^{\theta_{2}} e^{-\psi \operatorname{Im} z_{2}} d \psi \longrightarrow 0
$$

quando $s \rightarrow \infty$. Dado que o integrando somente tem um polo em $\lambda$, o Teorema do Resíduo garante que para um $\lambda \in \Gamma_{1}$ dado:

$$
-\frac{1}{2 \pi i} \int_{\Gamma_{2}} \frac{\mu^{z_{2}}}{\mu-\lambda} R(\lambda: A) x d \mu=\lim _{\mu \rightarrow \lambda} \mu^{z_{2}} R(\lambda: A) x=\lambda^{z_{2}} R(\lambda: A) x .
$$

Consequentemente, obtemos para $x \in X$ :

$$
A^{z_{1}} A^{z_{2}} x=-\frac{1}{2 \pi i} \int_{\Gamma_{1}} \lambda^{z_{1}} \lambda^{z_{2}} R(\lambda: A) x d \lambda=-\frac{1}{2 \pi i} \int_{\Gamma_{1}} \lambda^{z_{1}+z_{2}} R(\lambda: A) x d \lambda=A^{z_{1}+z_{2}} x \cdot \square
$$

Observação: No caso em que o exponente é um inteiro negativo, então a potência de um operador positivo $A$ é o produto apropriado de $A^{-1}$, i.e., 
Lema 1.21 Seja $A: D(A) \subset X \longrightarrow X$ um operador positivo de tipo $N \geq 1$. Para $n \in \mathbb{N}$ temos $A^{-n}=\left(A^{-1}\right)^{n}$, onde $A^{-1}$ denota a inversa de $A$.

Teorema 1.22 Seja $A: D(A) \subset X \longrightarrow X$ um operador positivo. Se $n \in \mathbb{N} e$ $z \in \mathbb{C}$ são dois números tais que $0<\operatorname{Re} z<n$, então:

$$
A^{-z}=\frac{\Gamma(n)}{\Gamma(n-z) \Gamma(z)} \int_{0}^{\infty} s^{-z+n-1} R(s:-A)^{n} d s
$$

Onde $\Gamma$ (.) representa a função Gamma.

Demonstração: Seja $A: D(A) \subset X \longrightarrow X$ um operador positivo de tipo $N \geq 1$. Primeiramente, vamos fazer um estudo do caso $n=1$. Portanto, suponha que $z \in \mathbb{C}$ é tal que $0<\operatorname{Re} z<1$. Sabemos que:

$$
A^{-z}=-\frac{1}{2 \pi i} \int_{\Gamma} \lambda^{-z} R(\lambda: A) d \lambda
$$

onde $\Gamma \subset \Sigma_{N}$ ( veja Lema 1.17) pode ser escolhido de tal modo que consiste em três segmentos:

$$
\left.\left\{-s e^{-i(\pi-\epsilon)}: s \in(-\infty, \delta]\right)\right\},\left\{\delta e^{i \psi}:|\psi| \leq \pi-\epsilon\right\},\left\{s e^{i(\pi-\epsilon)}: s \in[\delta, \infty)\right\}
$$

as quais são orientadas por suas parametrizações com $\epsilon>0$ e $\delta>0$ fixos e suficientemente pequenos. Segue assim, que:

$$
\begin{aligned}
A^{-z}= & -\frac{1}{2 \pi i} \int_{\infty}^{-\delta} e^{-(\ln |s|-i(\pi-\epsilon))} R\left(-s e^{-i(\pi-\epsilon)}: A\right)\left(-e^{-i(\pi-\epsilon)}\right) d s+ \\
& -\frac{1}{2 \pi i} \int_{-(\pi-\epsilon)}^{\pi-\epsilon} e^{-z(\ln \delta+i \psi)} R\left(\delta e^{i \psi}: A\right) \delta i e^{i \psi} d \psi+ \\
& -\frac{1}{2 \pi i} \int_{\delta}^{\infty} e^{-z(\ln s+i(\pi-\epsilon))} R\left(s e^{i(\pi-\epsilon)}: A\right) e^{i(\pi-\epsilon)} d s .
\end{aligned}
$$


Observe que para um $\delta>0$ dado, temos:

$$
\begin{aligned}
e^{-z(\ln (-s)-i(\pi-\epsilon))} & R\left(-s e^{-i(\pi-\epsilon)}: A\right)\left(-e^{-i(\pi-\epsilon)}\right) \\
& \longrightarrow e^{-z(\ln (-s)-i \pi)} R\left(-s e^{-i \pi}: A\right)\left(-e^{-i \pi}\right) \\
& =-(-s)^{-z} e^{i \pi z} R\left(-s e^{-i \pi}: A\right)\left(e^{-i \pi}\right) \\
& =-(-s)^{-z} e^{i \pi z}\left(-s e^{-i \pi} I-A\right)^{-1}\left(e^{-i \pi}\right) \\
& =-(-s)^{-z} e^{i \pi z}\left(-s e^{-i \pi} I+\left(e^{-i \pi}\right) A\right)^{-1} e^{-i \pi} \\
& =-(-s)^{-z} e^{i \pi z}\left(e^{-i \pi}[-s I+A]\right)^{-1} e^{-i \pi} \\
& =-(-s)^{-z} e^{i \pi z}(-s I+A)^{-1} \\
& =-(-s)^{-z} e^{i \pi z} R(-s:-A) .
\end{aligned}
$$

E também:

$$
\begin{gathered}
\left\|e^{-z(\ln |s|-i(\pi-\epsilon))} R\left(-s e^{-i(\pi-\epsilon)}: A\right)\left(-e^{-i(\pi-\epsilon)}\right)\right\| \leq e^{-\operatorname{Re} z \ln (-s)-\operatorname{Im} z(\pi-\epsilon)} \frac{2 N+1}{1-s} \\
\leq(2 N+1) e^{|\operatorname{Im} z| \pi}(-s)^{-\operatorname{Re} z-1},
\end{gathered}
$$

onde a função dominante é claramente integrável no intervalo $(-\infty,-\delta)$, pois Re $z>0$. Portanto, usando o Teorema da convergência dominada de Lebesgue obtemos:

$$
\begin{aligned}
\lim _{\epsilon \rightarrow 0} \int_{\infty}^{-\delta} e^{-(\ln |s|-i(\pi-\epsilon))} R\left(-s e^{-i(\pi-\epsilon)}: A\right)\left(-e^{-i(\pi-\epsilon)}\right) d s & \\
& =\int_{-\infty}^{-\delta}-(-s)^{-z} e^{i \pi z} R(-s:-A) d s
\end{aligned}
$$

Analogamente, para um $\delta>0$ dado, temos:

$$
e^{-z(\ln (s)+i(\pi-\epsilon))} R\left(s e^{i(\pi-\epsilon)}: A\right)\left(e^{i(\pi-\epsilon)}\right) \underset{\epsilon \rightarrow 0}{\longrightarrow} s^{-z} e^{-i \pi z} R(s:-A)
$$

$\mathrm{e}$

$$
\begin{gathered}
\left\|e^{-z(\ln s+i(\pi-\epsilon))} R\left(s e^{i(\pi-\epsilon)}: A\right) e^{i(\pi-\epsilon)}\right\| \leq e^{-\operatorname{Re} z \ln (s)+\operatorname{Im} z(\pi-\epsilon)} \frac{2 N+1}{1+s} \\
\leq(2 N+1) e^{|\operatorname{Im} z| \pi} s^{-\operatorname{Re} z-1}
\end{gathered}
$$

onde a função dominante é claramente integrável sobre $(\delta, \infty)$, já que $\operatorname{Re} z>0$. Novamente usando o Teorema da convergência dominada de Lebesgue, temos o seguinte resultado:

$$
\int_{\delta}^{\infty} e^{-z(\ln (s)+i(\pi-\epsilon))} R\left(s e^{i(\pi-\epsilon)}: A\right)\left(e^{i(\pi-\epsilon)}\right) d s \underset{\epsilon \rightarrow 0}{\longrightarrow} \int_{\delta}^{\infty} s^{-z} e^{-i \pi z} R(s:-A) d s
$$


Antes de continuar com o seguinte limite, precisamos de mais um resultado:

Teorema 1.23 Seja $f:[a, b) \longrightarrow X, a<b \leq \infty$, uma função tal que para qualquer $z \in[a, b)$ esta e integrável no intervalo $[a, z]$. Então a função $f$ é integrável sobre o intervalo $[a, b)$ se, e somente se, $\int_{a}^{b}\|f(t) d t\|<\infty$. Mais ainda, se $f$ é integrável em $[a, b)$, então temos:

$$
\int_{a}^{b} f(t) d t=\lim _{z \rightarrow b} \int_{a}^{z} f(t) d t
$$

Observa-se que:

$$
\int_{-\pi}^{\pi}\left\|e^{-z(\ln \delta+i \psi)} R\left(\delta e^{i \psi}: A\right) \delta i e^{i \psi}\right\| d \psi \leq \frac{2 N+1}{1+\delta} \delta^{1-\operatorname{Re} z} \int_{-\pi}^{\pi} e^{\psi \operatorname{Im} z} d \psi<\infty,
$$

Assim pelo teorema anteriormente mencionado,deduzimos que:

$$
\lim _{\epsilon \rightarrow 0} \int_{-(\pi-\epsilon)}^{\pi-\epsilon} e^{-z(\ln \delta+i \psi)} R\left(\delta e^{i \psi}: A\right) \delta i e^{i \psi} d \psi=\int_{-\pi}^{\pi} e^{-z(\ln \delta+i \psi)} R\left(\delta e^{i \psi}: A\right) \delta i e^{i \psi} d \psi
$$

Combinando (1.15),(1.16) e (1.17), chegamos a:

$$
\begin{aligned}
A^{-z} & =\frac{1}{2 \pi i} \int_{-\infty}^{-\delta}(-s)^{-z} e^{i \pi z} R(-s:-A) d s-\frac{1}{2 \pi i} \int_{-\pi}^{\pi} e^{-z(\ln \delta+i \psi)} R\left(\delta e^{i \psi}: A\right) \delta i e^{i \psi} d \psi \\
& -\frac{1}{2 \pi i} \int_{\delta}^{\infty} s^{-z} e^{-i \pi z} R(s:-A) d s
\end{aligned}
$$

E fazendo uma mudança de variáveis no primeiro termo:

$$
\begin{aligned}
A^{-z} & =\frac{1}{2 \pi i}\left(e^{i \pi z}-e^{-i \pi z}\right) \int_{\delta}^{\infty} s^{-z} R(s:-A) d s \\
& -\frac{1}{2 \pi i} \int_{-\pi}^{\pi} e^{-z(\ln \delta+i \psi)} R\left(\delta e^{i \psi}: A\right) \delta i e^{i \psi} d \psi
\end{aligned}
$$

Alem disso, a seguinte desigualdade também é válida:

$$
\left\|e^{-z(\ln \delta+i \psi)} R\left(\delta e^{i \psi}: A\right) \delta i e^{i \psi}\right\| \leq \frac{2 N+1}{1+\delta} \delta^{1-\operatorname{Re} z} e^{\operatorname{Im} z \psi} \underset{\delta \rightarrow 0}{\longrightarrow} 0,
$$

Pois Re $z<1$ (já que consideramos $n=1$ ). Portanto, aplicando o Teorema da convergência dominada de Lebesgue obtemos finalmente

$$
A^{-z}=\frac{1}{2 \pi i}\left(e^{i \pi z}-e^{-i \pi z}\right) \int_{0}^{\infty} s^{-z} R(s:-A) d s .
$$


Porém, para $z \in \mathbb{C}$ tal que $0<\operatorname{Re} z<1$, temos que:

$$
\Gamma(1-z) \Gamma(z)=\frac{\pi}{\sin \pi z}
$$

Com isso, o termo fora da integral anterior é igual a:

$$
\frac{1}{2 \pi i}\left(e^{i \pi z}-e^{-i \pi z}\right)=\frac{\sin \pi z}{\pi}=\frac{1}{\Gamma(1-z) \Gamma(z)},
$$

Pelo qual concluímos que:

$$
A^{-z}=\frac{1}{\Gamma(1-z) \Gamma(z)} \int_{0}^{\infty} s^{-z} R(s:-A) d s
$$

que prova o teorema para o caso $n=1$. Agora voltamos para o caso geral. Para este fim, seja $n \in \mathbb{N}$ e integra-se por partes $n-1$ vezes o resultado obtido no caso $0<\operatorname{Re} z<1$.Então, segue de (1.18) que

$$
\begin{aligned}
A^{-z} & =\frac{1}{\Gamma(1-z) \Gamma(z)} \frac{1}{1-z}\left[\left.s^{1-z} R(s:-A)\right|_{0} ^{\infty}+\int_{0}^{\infty} s^{-z+1} R(s:-A)^{2} d s\right] \\
& \vdots \\
& =\frac{1}{\Gamma(1-z) \Gamma(z)} \frac{(n-1) !}{(1-z)(2-z) \cdots(n-1-z)} \int_{0}^{\infty} s^{-z+n-1} R(s:-A)^{n} d s
\end{aligned}
$$

onde usamos primeiramente que:

$$
\frac{d^{n}}{d \lambda^{n}} R(\lambda: A)=(-1)^{n} n ! R(\lambda: A)^{n+1}, \lambda \in \rho(A) .
$$

e o fato de que para cada $k \in \mathbb{N}$

$$
\left\|s^{k-z} R(s:-A)^{k}\right\| \leq N^{k} s^{k-\operatorname{Re} z} \frac{1}{(1+s)^{k}} \longrightarrow 0
$$

quando $s$ se aproxima ao 0 ou $\infty$. Usando o fato que, $\Gamma(z+1)=z \Gamma(z-1)$, para $z \in \mathbb{C} \backslash\{0,-1,-2, \ldots\}$,segue que:

$$
\Gamma(n-z)=(n-1-z) \Gamma(n-1-z)=\ldots=(n-1-z) \cdots(1-z) \Gamma(1-z)
$$

Assim, temos:

$$
\frac{1}{\Gamma(1-z) \Gamma(z)} \frac{(n-1) !}{(1-z)(2-z) \cdots(n-1-z)}=\frac{\Gamma(n)}{\Gamma(n-z) \Gamma(z)} .
$$

Portanto, para $z \in \mathbb{C}$ tal que $0<\operatorname{Re} z<1$ obtemos:

$$
A^{-1}=\frac{\Gamma(n)}{\Gamma(n-z) \Gamma(z)} \int_{0}^{\infty} s^{-z+n-1} R(s:-A)^{n} d s, z \in \mathbb{C}
$$


onda a convergência da integral em (1.19) é provada ao detalhe em [14]. Assim, segue que para $z \in \mathbb{C}$ tal que $0<\operatorname{Re} z<n$ :

$$
A^{-1}=\frac{\Gamma(n)}{\Gamma(n-z) \Gamma(z)} \int_{0}^{\infty} s^{-z+n-1} R(s:-A)^{n} d s, z \in \mathbb{C}
$$

o qual completa a prova.

Corolário 1.24 Seja $A: D(A) \subset X \longrightarrow X$ um operador positivo. Então $A^{z}$ é um operador injetivo para Re $z<0$.

Prova: Fixa-se $z \in \mathbb{C}$ tal que $\operatorname{Re} z<0$. Suponha que $A^{z}=0$ para algum $x \in X$.

Seja $n \in \mathbb{N}$ de modo que $-n<\operatorname{Re} z$. Então o lema 1.23 nos garante que:

$$
A^{-n} z=A^{-n-z} A^{z} x=0 .
$$

Mas $A^{-n}$ é um operador injetivo. Logo $x=0$.

O Corolário anterior permite-nos definir potências fracionárias de um operador positivo com exponentes complexos que têm parte real positiva.

Definição 1.25 Seja $A: D(A) \subset X \longrightarrow X$ um operador positivo. Definimos:

$$
A^{z}=\left(A^{-z}\right)^{-1}: \operatorname{im}\left(A^{-z}\right) \subset X \longrightarrow X
$$

para $z \in \mathbb{C}$ tal que Re $z>0$.

Finalmente, vamos dar um teorema que fornece uma maneira de re-escrever aqueles operadores anteriormente definidos.

Teorema 1.26 Seja $A: D(A) \subset X \longrightarrow X$ um operador positivo de tipo $N \geq 1$. Se $n \in \mathbb{N}$ e $z \in \mathbb{C}$ são tal que $0<\operatorname{Re} z<n$, então:

$$
A^{z}=\frac{\Gamma(n)}{\Gamma(n-z) \Gamma(z)} \int_{0}^{\infty} s^{z-1} A^{n} R(s:-A)^{n} x d s, x \in D\left(A^{n}\right) .
$$

Demonstração: Aplicando o Teorema 1.22 com $n-z$ em vez de $z$ obtemos:

$$
A^{z-n}=A^{-(n-z)}=\frac{\Gamma(n)}{\Gamma(n-z) \Gamma(z)} \int_{0}^{\infty} s^{z-1} R(s:-A)^{n} x d s, x \in X .
$$

Fixe $x \in D\left(A^{n}\right)$ e note que as funções:

$$
s \mapsto s^{z-1} R(s:-A)^{n} x \quad \text { e } \quad s \mapsto s^{z-1} A^{n} R(s:-A)^{n} x=s^{z-1} R(s:-A)^{n} A^{n} x
$$


são funções bem definidas, contínuas e integráveis sobre $(0, \infty)$, pois

$$
\begin{aligned}
\int_{0}^{\infty}\left\|s^{z-1} R(s:-A)^{n} y\right\| d s & \leq N^{n}\|y\| \int_{0}^{\infty} s^{\operatorname{Re} z-1} \frac{1}{(1+s)^{n}} d s \\
& \leq N^{n}\|y\|\left(\int_{0}^{1} s^{\operatorname{Re} z-1} d s+\int_{1}^{\infty} s^{\operatorname{Re} z-n-1} d s\right) \\
& =N^{n}\|y\|\left(\frac{1}{\operatorname{Re} z}+\frac{1}{n-\operatorname{Re} z}\right), y \in X .
\end{aligned}
$$

Finalmente, da propriedade de fechamento de $A^{n}$ e com as hipóteses anteriores, obtemos:

$$
\begin{aligned}
A^{n} A^{z-n} x & =A^{n}\left(\frac{\Gamma(n)}{\Gamma(n-z) \Gamma(z)} \int_{0}^{\infty} s^{z-1} R(s:-A)^{n} x d s\right) \\
& =\frac{\Gamma(n)}{\Gamma(n-z) \Gamma(z)} \int_{0}^{\infty} s^{z-1} A^{n} R(s:-A)^{n} x d s, x \in D\left(A^{n}\right) .
\end{aligned}
$$

Assim, (1.20) é provado. 


\section{Capítulo 2}

\section{Estimativas de operadores elípticos em $\mathbb{R}^{n}$}

Seja $A=\left(a_{i, j}(x)\right)$ uma matriz complexa de ordem $n$ com coeficientes $a_{i, j} \in$ $L^{\infty}\left(\mathbb{R}^{n}, \mathbb{C}\right)$, satisfazendo a condição de elipticidade (ou 'acretividade'):

$$
\lambda|\xi|^{2} \leq \operatorname{Re} A \xi \cdot \xi^{*} \quad \text { e } \quad\left|A \xi \cdot \zeta^{*}\right| \leq \Lambda|\xi||\zeta|,
$$

para todos $\xi, \zeta \in \mathbb{C}^{n}$ e para algumas constantes $\lambda, \Lambda$ tal que $0<\lambda \leq \Lambda<\infty$. Aqui, $u \cdot v=u_{1} v_{1}+\cdots+u_{n} v_{n}$ e $u \cdot v^{*}=u_{1} \overline{v_{1}}+\cdots+u_{n} \overline{v_{n}}$, e, além disso, $A \xi \cdot \zeta^{*}=\sum_{j, k=1}^{n} a_{j, k}(x) \xi_{k} \bar{\zeta}_{j}$

Eventualmente vamos considerar funções a valores em $\mathbb{C}^{n}$, e neste caso consideraremos letras em negrito (por exemplo $\mathbf{f}=\left(f_{1}, \cdots, f_{n}\right)$ ).

Considere $\mathfrak{a}: H^{1}\left(\mathbb{R}^{n}\right) \times H^{1}\left(\mathbb{R}^{n}\right) \rightarrow \mathbb{C}$ a forma sesquilinear definida por

$$
\mathfrak{a}(f, g)=\int_{\mathbb{R}^{n}} A \nabla f \cdot \overline{\nabla g} d x .
$$

Então a é claramente densamente definida em $L^{2}\left(\mathbb{R}^{n}\right)$. Além disso, por (2.1) também é contínua e acretiva.

Seja $\tilde{L}: D(\tilde{L}) \subset L^{2}\left(\mathbb{R}^{n}\right) \rightarrow L^{2}\left(\mathbb{R}^{n}\right)$ o operador associado a $\mathfrak{a}$. No que segue denotaremos por

$$
L=-\operatorname{div}(A \nabla),
$$

o fecho (em $\left.L^{2}\left(\mathbb{R}^{n}\right)\right)$ do operador $\tilde{L}$.

Neste Capítulo vamos derivar algumas estimativas correspondentes ao operador $L$. 


\section{CAPÍTULO 2. ESTIMATIVAS DE OPERADORES ELÍPTICOS EM $\mathbb{R}^{N}$}

Dado $t \in \mathbb{R}$, de (2.1) é fácil ver que $I+t^{2} L$ é injetor, e do Teorema de LaxMilgran que também é sobrejetor. Dessa forma ficam bem definidos os operadores dados no próximo lema:

Lema 2.1 Sejam $E$ e $F$ dois conjuntos fechados em $\mathbb{R}^{n}$ e denote por $d=\operatorname{dist}(E, F)$. Então têm-se as seguintes desigualdades:

$$
\begin{gathered}
\int_{F}\left|\left(I+t^{2} L\right)^{-1} f(x)\right|^{2} d x \leq C e^{-\frac{d}{c t}} \int_{E}|f(x)|^{2} d x, \quad \text { supp } f \subset E . \\
\int_{F}\left|t \nabla\left(I+t^{2} L\right)^{-1} f(x)\right|^{2} d x \leq C e^{-\frac{d}{c t}} \int_{E}|f(x)|^{2} d x, \quad \text { supp } f \subset E . \\
\int_{F}\left|\left(I+t^{2} L\right)^{-1} t \operatorname{div} \mathbf{f}(x)\right|^{2} d x \leq C e^{-\frac{d}{c t}} \int_{E}|f(x)|^{2} d x, \quad \text { supp } \mathbf{f} \subset E .
\end{gathered}
$$

onde $c>0$ depende apenas de $\lambda$ e $\Lambda$, e $C$ de $n, \lambda$ e $\Lambda$.

Demonstração: Note que pela continuidade dos operadores $\left(I+t^{2} L\right)^{-1}, t \nabla(I+$ $\left.t^{2} L\right)^{-1},\left(I+t^{2} L\right)^{-1} t$ div e $t^{2} \nabla\left(I+t^{2} L\right)^{-1}$ div, é suficiente provar o caso $d>0$. Comparando as constantes também vemos que é suficiente provar o caso $d \geq t>$ 0 .

Começamos considerando $u_{t}=\left(I+t^{2} L\right)^{-1} f$. Assim, para todo $v \in H^{1}\left(\mathbb{R}^{n}\right)$,

$$
\begin{aligned}
\left(I+t^{2} L\right) u_{t}=f & \Rightarrow u_{t}-t^{2} \operatorname{div}\left(A \nabla u_{t}\right)=f \\
& \Rightarrow u_{t} \cdot v-t^{2} \operatorname{div}(A \nabla u) \cdot v=f \cdot v
\end{aligned}
$$

Logo:

$$
\int_{\mathbb{R}^{n}} u_{t} v d x-\int_{\mathbb{R}^{n}} t^{2} \operatorname{div}\left(A \nabla u_{t}\right) v d x=\int_{\mathbb{R}^{n}} f v d x
$$

Da formula de Green, temos que:

$$
-\int_{\mathbb{R}^{n}} \operatorname{div}\left(A \nabla u_{t}\right) v d x=\int_{\mathbb{R}^{n}} A \nabla u_{t} \cdot \nabla v d x
$$

Pelo qual, obtemos:

$$
\int_{\mathbb{R}^{n}} u_{t} v d x+t^{2} \int_{\mathbb{R}^{n}} A \nabla u_{t} \cdot \nabla v d x=\int_{\mathbb{R}^{n}} f v d x .
$$

Agora, tome-se $v=\overline{u_{t}} \eta^{2}$, onde $\eta$ é uma função positiva que têm as seguintes propriedades: $\eta \in C_{0}^{\infty}\left(\mathbb{R}^{n}\right)$, supp $\eta \subset E^{c},\|\nabla \eta\|_{\infty} \sim 1 / d, \eta \equiv 1$ sobre $F, \eta(x) \equiv 0$, 
para todo $x$ tal que $\operatorname{dist}(x, E) \leq d / 2$, e usando a hipótese de que $\operatorname{supp} f \subset E$ :

$$
\begin{aligned}
\int_{\mathbb{R}^{n}} u_{t}\left(\overline{u_{t}} \eta^{2}\right) d x+t^{2} \int_{\mathbb{R}^{n}} A \nabla u_{t} \cdot \nabla\left(\overline{u_{t}} \eta^{2}\right) d x & =\int_{\mathbb{R}^{n}} f \cdot \overline{u_{t}} \cdot \eta^{2} d x \\
& =\int_{E} f \cdot \overline{u_{t}} \cdot \eta^{2} d x+\int_{E^{c}} f \cdot \overline{u_{t}} \cdot \eta^{2} d x \\
& =0
\end{aligned}
$$

Por outra parte, do lado esquerdo da identidade anterior é igual a:

$$
\begin{aligned}
\int_{\mathbb{R}^{n}}\left|u_{t}\right|^{2} \eta^{2} d x+t^{2} \int_{\mathbb{R}^{n}} A \nabla u_{t} \cdot \nabla\left(\overline{u_{t}} \eta^{2}\right) d x & =\int_{\mathbb{R}^{n}}\left|u_{t}\right|^{2} \eta^{2} d x+t^{2} \int_{\mathbb{R}^{n}} A \nabla u_{t} \cdot\left(\nabla \overline{u_{t}} \eta^{2}\right) d x+ \\
& t^{2} \int_{\mathbb{R}^{n}} A \nabla u_{t} \cdot\left(\overline{u_{t}} \nabla \eta^{2}\right) d x \\
& =\int_{\mathbb{R}^{n}}\left|u_{t}\right|^{2} \eta^{2} d x+t^{2} \int_{\mathbb{R}^{n}} A \nabla u_{t} \cdot\left(\nabla \overline{u_{t}} \eta^{2}\right) d x+ \\
& t^{2} \int_{\mathbb{R}^{n}} A \nabla u_{t} \cdot\left(\overline{u_{t}} \nabla \eta^{2}\right) d x \\
& =\int_{\mathbb{R}^{n}}\left|u_{t}\right|^{2} \eta^{2} d x+t^{2} \iint_{\mathbb{R}^{n}} A \nabla u_{t} \cdot\left(\nabla \overline{u_{t}} \eta^{2}\right) d x+ \\
& t^{2} \int_{\mathbb{R}^{n}} A \nabla u_{t} \cdot\left(\overline{u_{t}} \nabla \eta .2 \eta\right) d x
\end{aligned}
$$

Por tanto, juntando os dois resultados, e escrevendo a função $\eta$ dentro da matriz $A$ temos que:

$$
\begin{aligned}
& \int_{\mathbb{R}^{n}}\left|u_{t}\right|^{2} \eta^{2} d x+t^{2} \int_{\mathbb{R}^{n}} A \nabla u_{t} \cdot\left(\nabla \overline{u_{t}} \eta^{2}\right) d x+t^{2} \int_{\mathbb{R}^{n}} A\left(\eta \nabla u_{t}\right) \cdot\left(\overline{u_{t}} \nabla \eta .2\right) d x=0 \\
\Rightarrow & \\
& \int_{\mathbb{R}^{n}}\left|u_{t}\right|^{2} \eta^{2} d x+t^{2} \int_{\mathbb{R}^{n}} A \nabla u_{t} \cdot\left(\nabla \overline{u_{t}} \eta^{2}\right) d x=-2 t^{2} \int_{\mathbb{R}^{n}} A\left(\eta \nabla u_{t}\right) \cdot\left(\overline{u_{t}} \nabla \eta\right) d x
\end{aligned}
$$

Uma importante observação na equação (2.3) é o fato de que $\eta$ é uma função real positiva e que $\overline{\nabla u_{t}}=\nabla \overline{u_{t}}$. Dessa maneira, (2.3) é reescrito como:

$$
\int_{\mathbb{R}^{n}}\left|u_{t}\right|^{2} \eta^{2} d x+t^{2} \int_{\mathbb{R}^{n}} A \nabla u_{t} \cdot \overline{\nabla u_{t}} \eta^{2} d x=-2 t^{2} \int_{\mathbb{R}^{n}} A\left(\eta \nabla u_{t}\right) \cdot \overline{u_{t} \nabla \eta} d x
$$


Tomando parte real em ambos termos em (2.4):

$$
\begin{aligned}
\int_{\mathbb{R}^{n}}\left|u_{t}\right|^{2} \eta^{2} d x+t^{2} \operatorname{Re} \int_{\mathbb{R}^{n}} A \nabla u_{t} \cdot \overline{\nabla u_{t}} \eta^{2} d x & =-2 t^{2} \operatorname{Re} \int_{\mathbb{R}^{n}} A\left(\eta \nabla u_{t}\right) \cdot \overline{u_{t} \nabla \eta} d x \\
\Rightarrow \quad & \\
\int_{\mathbb{R}^{n}}\left|u_{t}\right|^{2} \eta^{2} d x+t^{2} \int_{\mathbb{R}^{n}} \operatorname{Re} A \nabla u_{t} \cdot \overline{\nabla u_{t}} \eta^{2} d x & =-2 t^{2} \operatorname{Re} \int_{\mathbb{R}^{n}} A\left(\eta \nabla u_{t}\right) \cdot \overline{u_{t} \nabla \eta} d x \\
& \leq\left|-2 t^{2} \int_{\mathbb{R}^{n}} A\left(\eta \nabla u_{t}\right) \cdot \overline{u_{t} \nabla \eta} d x\right| \\
& \leq 2 t^{2} \int_{\mathbb{R}^{n}}\left|A\left(\eta \nabla u_{t}\right) \cdot \overline{u_{t} \nabla \eta}\right| d x
\end{aligned}
$$

Aqui, usamos a primeira condição de elipticidade no segundo termo do lado esquerdo; e a segunda condição no lado direito da anterior equação, obtendo assim:

$$
\int_{\mathbb{R}^{n}}\left|u_{t}\right|^{2} \eta^{2} d x+\lambda t^{2} \int_{\mathbb{R}^{n}}\left|\nabla u_{t}\right|^{2} \eta^{2} d x \leq 2 t^{2} \Lambda \int_{\mathbb{R}^{n}}\left|\eta \nabla u_{t}\right|\left|u_{t} \nabla \eta\right| d x
$$

Usando a desigualdade $2|a b| \leq \varepsilon|a|^{2}+\varepsilon^{-1}|b|^{2}$, para todo $\varepsilon>0$, com $a=\left|\eta \nabla u_{t}\right|$ e $b=\left|u_{t} \nabla \eta\right|$ :

$$
\int_{\mathbb{R}^{n}}\left|u_{t}\right|^{2} \eta^{2} d x+\lambda t^{2} \int_{\mathbb{R}^{n}}\left|\nabla u_{t}\right|^{2} \eta^{2} d x \leq t^{2} \Lambda \varepsilon \int_{\mathbb{R}^{n}}\left|\nabla u_{t}\right|^{2} \eta^{2} d x+t^{2} \Lambda \varepsilon^{-1} \int_{\mathbb{R}^{n}}\left|u_{t}\right|^{2}|\nabla \eta|^{2} d x
$$

Tomando-se $\varepsilon=\frac{\lambda}{\Lambda}$, a desigualdade anterior é equivalente a:

$$
\begin{gathered}
\int_{\mathbb{R}^{n}}\left|u_{t}\right|^{2} \eta^{2} d x+\lambda t^{2} \int_{\mathbb{R}^{n}}\left|\nabla u_{t}\right|^{2} \eta^{2} d x \leq t^{2} \lambda \int_{\mathbb{R}^{n}}\left|\nabla u_{t}\right|^{2} \eta^{2} d x+\frac{t^{2} \Lambda^{2}}{\lambda} \int_{\mathbb{R}^{n}}\left|u_{t}\right|^{2}|\nabla \eta|^{2} d x \\
\Rightarrow \\
\int_{\mathbb{R}^{n}}\left|u_{t}\right|^{2} \eta^{2} d x \leq \frac{t^{2} \Lambda^{2}}{\lambda} \int_{\mathbb{R}^{n}}\left|u_{t}\right|^{2}|\nabla \eta|^{2} d x .
\end{gathered}
$$

Substituindo $\eta$ por $e^{\alpha \theta}-1$, onde $0<\theta(x), \operatorname{supp} \theta \subset E^{c},\|\theta\|_{\infty} \leq 1,\|\nabla \theta\|_{\infty} \sim 1 / d$ e o valor de $\alpha$ é igual a

$$
\alpha=\frac{\sqrt{\lambda}}{2 \Lambda t\|\nabla \theta\|_{\infty}}
$$


a equação (2.5) se transforma em:

$$
\begin{aligned}
\int_{\mathbb{R}^{n}}\left|u_{t}\right|^{2}\left|e^{\alpha \theta}-1\right|^{2} d x & \leq \frac{t^{2} \Lambda^{2}}{\lambda} \int_{\mathbb{R}^{n}}\left|u_{t}\right|^{2}\left|\nabla\left(e^{\alpha \theta}-1\right)\right|^{2} d x \\
& =\frac{t^{2} \Lambda^{2}}{\lambda} \int_{\mathbb{R}^{n}}\left|u_{t}\right|^{2}\left|\nabla e^{\alpha \theta}\right|^{2} d x \\
& =\frac{t^{2} \Lambda^{2}}{\lambda} \int_{\mathbb{R}^{n}}\left|u_{t}\right|^{2}\left|\alpha e^{\alpha \theta} \nabla \theta\right|^{2} d x \\
& =\frac{t^{2} \Lambda^{2} \alpha^{2}}{\lambda} \int_{\mathbb{R}^{n}}\left|u_{t}\right|^{2}\left|e^{\alpha \theta}\right|^{2}|\nabla \theta|^{2} d x \\
& =\frac{1}{4\|\nabla \theta\|_{\infty}^{2}} \int_{\mathbb{R}^{n}}\left|u_{t}\right|^{2}\left|e^{\alpha \theta}\right|^{2}|\nabla \theta|^{2} d x \\
& \leq \frac{1}{4} \int_{\mathbb{R}^{n}}\left|u_{t}\right|^{2}\left|e^{\alpha \theta}\right|^{2} d x
\end{aligned}
$$

Por outra parte, tomando o lado esquerdo da desigualdade anterior e multiplicando por 4:

$$
\begin{aligned}
4 \int_{\mathbb{R}^{n}}\left|u_{t}\right|^{2}\left|e^{\alpha \theta}-1\right|^{2} d x & \geq 4 \int_{\mathbb{R}^{n}}\left|u_{t}\right|^{2}\left(\left|e^{\alpha \theta}\right|-|1|\right)^{2} d x \\
& \geq 4 \int_{\mathbb{R}^{n}}\left|u_{t}\right|^{2}\left(\left|e^{\alpha \theta}\right|^{2}-2\left|e^{\alpha \theta}\right| 1+1\right) d x \\
& =4 \int_{\mathbb{R}^{n}}\left|u_{t}\right|^{2}\left|e^{\alpha \theta}\right|^{2} d x-4 \int_{\mathbb{R}^{n}} 2\left|u_{t}\right|^{2}\left|e^{\alpha \theta}\right| d x+4 \int_{\mathbb{R}^{n}}\left|u_{t}\right|^{2} d x
\end{aligned}
$$

Dessa maneira, podemos escrever:

$$
\begin{gathered}
4 \int_{\mathbb{R}^{n}}\left|u_{t}\right|^{2}\left|e^{\alpha \theta}\right|^{2} d x+4 \int_{\mathbb{R}^{n}}\left|u_{t}\right|^{2} d x \leq \int_{\mathbb{R}^{n}}\left|u_{t}\right|^{2}\left|e^{\alpha \theta}\right|^{2} d x+4 \int_{\mathbb{R}^{n}} 2\left|u_{t}\right|^{2}\left|e^{\alpha \theta}\right| d x \\
3 \int_{\mathbb{R}^{n}}\left|u_{t}\right|^{2}\left|e^{\alpha \theta}\right|^{2} d x \leq 4 \int_{\mathbb{R}^{n}} 2\left|u_{t}\right|^{2}\left|e^{\alpha \theta}\right| d x-4 \int_{\mathbb{R}^{n}}\left|u_{t}\right|^{2} d x
\end{gathered}
$$

Usando novamente $2|a b| \leq \varepsilon|a|^{2}+\varepsilon^{-1}|b|^{2}$, com $a=\left|e^{\alpha \theta}\right|$ e $b=1$, tem-se:

$$
\begin{aligned}
3 \int_{\mathbb{R}^{n}}\left|u_{t}\right|^{2}\left|e^{\alpha \theta}\right|^{2} d x & \leq 4 \int_{\mathbb{R}^{n}}\left|u_{t}\right|^{2}\left(\varepsilon\left|e^{\alpha \theta}\right|^{2}+\varepsilon^{-1}\right) d x-4 \int_{\mathbb{R}^{n}}\left|u_{t}\right|^{2} d x \\
& =4 \varepsilon \int_{\mathbb{R}^{n}}\left|u_{t}\right|^{2}\left|e^{\alpha \theta}\right|^{2} d x+4 \varepsilon^{-1} \int_{\mathbb{R}^{n}}\left|u_{t}\right|^{2} d x-4 \int_{\mathbb{R}^{n}}\left|u_{t}\right|^{2} d x
\end{aligned}
$$


28 CAPÍTULO 2. ESTIMATIVAS DE OPERADORES ELÍPTICOS EM $\mathbb{R}^{N}$

$\Rightarrow$

$$
(3-4 \varepsilon) \int_{\mathbb{R}^{n}}\left|u_{t}\right|^{2}\left|e^{\alpha \theta}\right|^{2} d x \leq\left(4 \varepsilon^{-1}-4\right) \int_{\mathbb{R}^{n}}\left|u_{t}\right|^{2} d x
$$

Como a escolha de $\varepsilon$ é arbitrária, em particular consideramos $\varepsilon=\frac{1}{2}$, e reduzimos a desigualdade a:

$$
\begin{aligned}
\int_{\mathbb{R}^{n}}\left|u_{t}\right|^{2}\left|e^{\alpha \theta}\right|^{2} d x & \leq 4 \int_{\mathbb{R}^{n}}\left|u_{t}\right|^{2} d x \\
& =4 \int_{\mathbb{R}^{n}}\left|\left(I+t^{2} L\right)^{-1} f(x)\right|^{2} d x \\
& \leq 4 \int_{\mathbb{R}^{n}}|f(x)|^{2} d x=4 \int_{E}|f(x)|^{2} d x
\end{aligned}
$$

onde usamos o resultado do Teorema 1.13 e que supp $f \subset E$.

Assuma agora mais uma condição para $\theta$ : suponha que $\theta \equiv 1$, sobre o conjunto F. Então:

$$
\int_{\mathbb{R}^{n}}\left|u_{t}\right|^{2}\left|e^{\alpha \theta}\right|^{2} d x \geq \int_{F}\left|u_{t}\right|^{2}\left|e^{\alpha \theta}\right|^{2} d x=\int_{F}\left|u_{t}\right|^{2}\left|e^{\alpha}\right|^{2} d x=e^{2 \alpha} \int_{F}\left|u_{t}\right|^{2} d x
$$

Logo, substituindo essa limitação na penúltima equação, chegamos a:

$$
\int_{F}\left|\left(I+t^{2} L\right)^{-1} f(x)\right|^{2} d x=\int_{F}\left|u_{t}\right|^{2} d x \leq 4 e^{-2 \alpha} \int_{E}|f(x)|^{2} d x
$$

Para achar as constantes $C$ e $c$, de maneira que dependam somente das variáveis mencionadas anteriormente, basta substituir o valor de $\alpha$, ou seja:

$$
4 e^{-2 \alpha}=4 e^{-\frac{2 \sqrt{\lambda}}{2 \Lambda t\|\nabla \theta\|_{\infty}}}=4 e^{-\frac{\sqrt{\lambda}}{\Lambda t\|\nabla \theta\|_{\infty}}}
$$

Como $\|\nabla \theta\|_{\infty} \sim 1 / d$ então:

$$
4 e^{-2 \alpha}=4 e^{-\frac{\sqrt{\lambda}}{\Lambda t \frac{1}{d}}}=4 e^{-\frac{\sqrt{\lambda} d}{\Lambda t}}=C e^{-\frac{d}{c t}}
$$

onde $c=\frac{\Lambda}{\sqrt{\lambda}}$ e $C=4$. Finalmente, concluímos que:

$$
\int_{F}\left|\left(I+t^{2} L\right)^{-1} f(x)\right|^{2} d x \leq C e^{-\frac{d}{c t}} \int_{E}|f(x)|^{2} d x .
$$

Com isso, provamos a primeira desigualdade.

Para demonstrar a seguinte, basta observar que, antes de obter a equação $(2,5)$, em vez de usar $\varepsilon=\frac{\lambda}{\Lambda}$, usamos $\varepsilon=\frac{\lambda}{2 \Lambda}$, com o qual temos:

$$
\int_{\mathbb{R}^{n}}\left|u_{t}\right|^{2} \eta^{2} d x+\lambda t^{2} \int_{\mathbb{R}^{n}}\left|\nabla u_{t}\right|^{2} \eta^{2} d x \leq \frac{t^{2} \lambda}{2} \int_{\mathbb{R}^{n}}\left|\nabla u_{t}\right|^{2} \eta^{2} d x+\frac{2 t^{2} \Lambda^{2}}{\lambda} \int_{\mathbb{R}^{n}}\left|u_{t}\right|^{2}|\nabla \eta|^{2} d x
$$


aqui, o lado esquerdo desta equação é maior o igual que um de seus termos, pelo qual tem-se que:

$$
\frac{t^{2} \lambda}{2} \int_{\mathbb{R}^{n}}\left|\nabla u_{t}\right|^{2} \eta^{2} d x \leq \frac{2 t^{2} \Lambda^{2}}{\lambda} \int_{\mathbb{R}^{n}}\left|u_{t}\right|^{2}|\nabla \eta|^{2} d x
$$

Com as hipóteses sobre $\eta$, essa desigualdade pode ser escrita como

$$
\int_{F}\left|t \nabla u_{t}\right|^{2} d x \leq \int_{\mathbb{R}^{n}}\left|t \nabla u_{t}\right|^{2} \eta^{2} d x \leq \frac{4 t^{2} \Lambda^{2}}{\lambda^{2}} \int_{\mathbb{R}^{n}}\left|u_{t}\right|^{2}|\nabla \eta|^{2} d x
$$

Denote por $\bar{F}=: \operatorname{supp}(\eta)$. Observa-se que, pela definição de $\eta, F \subset \bar{F}, \bar{F} \neq \emptyset$ e $d(\bar{F}, E) \geq 1 / 2>0$. Com isso, podemos aplicar a primeira desigualdade aqui, para obter

$$
\begin{aligned}
\int_{F}\left|t \nabla u_{t}\right|^{2} d x & \leq \frac{4 t^{2} \Lambda^{2}}{\lambda^{2}} \int_{\mathbb{R}^{n}}\left|u_{t}\right|^{2}|\nabla \eta|^{2} d x \\
& \leq \frac{4 t^{2} \Lambda^{2}}{\lambda^{2}} \int_{\bar{F}}\left|u_{t}\right|^{2}|\nabla \eta|^{2} d x \\
& \leq \frac{4 n t^{2} \Lambda^{2}}{\lambda^{2}} \int_{\bar{F}}\left|u_{t}\right|^{2}\|\nabla \eta\|_{\infty}^{2} d x \\
& \leq \frac{C t^{2}}{d^{2}} \int_{\bar{F}}\left|u_{t}\right|^{2} d x \\
& \leq C e^{-\frac{d}{c t}} \int_{E}|f(x)|^{2} d x
\end{aligned}
$$

E concluímos que:

$$
\int_{F}\left|t \nabla\left(I+t^{2} L\right)^{-1} f(x)\right|^{2} d x \leq C e^{-\frac{d}{c t}} \int_{E}|f(x)|^{2} d x
$$

onde $C$ depende de $n, \lambda$, e $\Lambda$ e $c>0$ apenas de $\lambda$ e $\Lambda$.

Para fazer a terceira desigualdade, vamos usar a segunda, aplicando operadores 
adjuntos. Isto é, dada uma função $g \in L^{2}\left(\mathbb{R}^{n}\right)$, têm-se:

$$
\begin{aligned}
\int_{\mathbb{R}^{n}}\left(I+t^{2} L\right)^{-1} t \eta \operatorname{div} \mathbf{f} \bar{g} d x & =\left\langle\left(I+t^{2} L\right)^{-1} t \operatorname{div} \mathbf{f}, \eta g\right\rangle=\left\langle t \operatorname{div} \mathbf{f},\left(\left(I+t^{2} L\right)^{-1}\right)^{*} \eta g\right\rangle \\
& =\int_{\mathbb{R}^{n}} t \operatorname{div} \mathbf{f}\left(\left(I+t^{2} L\right)^{-1}\right)^{*} \eta \bar{g} d x \\
& =\int_{\mathbb{R}^{n}} t \operatorname{div} \mathbf{f}\left(\left(I+t^{2} L\right)^{*}\right)^{-1} \eta \bar{g} d x \\
& =\int_{\mathbb{R}^{n}}-t \mathbf{f} \nabla\left(\left(I+t^{2} L^{*}\right)\right)^{-1} \eta \bar{g} d x
\end{aligned}
$$

Logo, temos que

$$
\begin{aligned}
\left|\int_{\mathbb{R}^{n}}\left(I+t^{2} L\right)^{-1} t \eta \operatorname{div} \mathbf{f} \bar{g} d x\right| & \leq \int_{\mathbb{R}^{n}}|\mathbf{f}|\left|t \nabla\left(\left(I+t^{2} L^{*}\right)\right)^{-1} \eta \bar{g}\right| d x \\
& \leq \int_{\mathbb{R}^{n}}|\mathbf{f}|\left|t \nabla\left(\left(I+t^{2} L^{*}\right)\right)^{-1} \eta \bar{g}\right| d x \\
& \leq\left(\int_{E}|\mathbf{f}|^{2} d x\right)^{\frac{1}{2}}\left(\int_{E}\left|t \nabla\left(\left(I+t^{2} L^{*}\right)\right)^{-1} \eta \bar{g}\right|^{2} d x\right)^{\frac{1}{2}}
\end{aligned}
$$

Considere $\eta$ como nas outras demonstrações, com $\tilde{F}=\operatorname{supp} \eta,\|\eta\|_{\infty} \leq C$. Então $\operatorname{supp}(\eta g) \subset \tilde{F}$, e $d(\tilde{F}, E) \geq d / 2$. Dessa maneira, pode-se aplicar a segunda desigualdade trocando E e $\tilde{F}$, para o nosso operador $t \nabla\left(\left(I+t^{2} L^{*}\right)\right)^{-1}$, obtendo:

$$
\begin{aligned}
\left|\int_{\mathbb{R}^{n}}\left(I+t^{2} L\right)^{-1} t \eta \operatorname{div} \mathbf{f} \bar{g} d x\right| & \leq\left(\int_{E}|\mathbf{f}(x)|^{2} d x\right)^{\frac{1}{2}}\left(C e^{-\frac{d}{c t}} \int_{\tilde{\mathrm{F}}}|\eta \bar{g}(x)|^{2} d x\right)^{\frac{1}{2}} \\
& \leq\left(C e^{-\frac{d}{c t}} \int_{E}|\mathbf{f}(x)|^{2} d x\right)^{\frac{1}{2}}\left(\|\eta\|_{\infty}^{2} \int_{\tilde{\mathrm{F}}}|\bar{g}(x)|^{2} d x\right)^{\frac{1}{2}} \\
& \leq\left(C e^{-\frac{d}{c t}} \int_{E}|\mathbf{f}(x)|^{2} d x\right)^{\frac{1}{2}}\left(\int_{\mathbb{R}^{n}}|\bar{g}(x)|^{2} d x\right)^{\frac{1}{2}}
\end{aligned}
$$

Portanto, chegamos a:

$$
\left|\int_{\mathbb{R}^{n}}\left(I+t^{2} L\right)^{-1} t \eta \operatorname{div} \mathbf{f} \bar{g} d x\right| \leq\left(C e^{-\frac{d}{c t}} \int_{E}|\mathbf{f}(x)|^{2} d x\right)^{\frac{1}{2}}\left(\int_{\mathbb{R}^{n}}|\bar{g}(x)|^{2} d x\right)^{\frac{1}{2}}
$$


Escolha $g(x)=\left(I+t^{2} L\right)^{-1} t \operatorname{div} \mathbf{f}(x) \eta$, para obter:

$\int_{\mathbb{R}^{n}}\left|\left(I+t^{2} L\right)^{-1} t \operatorname{div} \mathbf{f} \eta\right|^{2} d x \leq\left(C e^{-\frac{d}{c t}} \int_{E}|\mathbf{f}(x)|^{2} d x\right)^{\frac{1}{2}}\left(\int_{\mathbb{R}^{n}}\left|\left(I+t^{2} L\right)^{-1} t \operatorname{div} \mathbf{f} \eta\right|^{2} 2 d x\right)^{\frac{1}{2}}$

onde nós deduzimos que:

$$
\int_{\mathbb{R}^{n}}\left|\left(I+t^{2} L\right)^{-1} t \operatorname{div} \mathbf{f}(x) \eta\right|^{2} d x \leq C e^{-\frac{d}{c t}} \int_{E}|\mathbf{f}(x)|^{2} d x
$$

Finalmente observa-se que a integral sobre $\mathbb{R}^{n}$ e maior o igual que a integral sobre o conjunto $F$, onde a função $\eta$ é igual a 1 . Isto é:

$$
\int_{F}\left|\left(I+t^{2} L\right)^{-1} t \operatorname{div} \mathbf{f}(x)\right|^{2} d x \leq C e^{-\frac{d}{c t}} \int_{E}|\mathbf{f}(x)|^{2} d x
$$

onde $C$ depende apenas de $n, \lambda$ e $\Lambda$ e $c>0$, de $\lambda$, $\Lambda$. Com isso, queda feita a demonstração da terceira desigualdade e finaliza a prova do lema.

Lema 2.2 Para qualquer função Lipschitz $f$ e $t>0$, têm-se:

$$
\begin{gathered}
\left\|\left[\left(I+t^{2} L\right)^{-1}, f\right]\right\|_{o p} \leq C t\|\nabla f\|_{\infty} \\
\left\|\nabla\left[\left(I+t^{2} L\right)^{-1}, f\right]\right\|_{o p} \leq C\|\nabla f\|_{\infty}
\end{gathered}
$$

Onde $C$ depende apenas de $n, \lambda$ e $\Lambda$. Aqui, \|\|$_{o p}$ denota a norma de um operador agindo de $L^{2}\left(\mathbb{R}^{n}\right)$ à $L^{2}\left(\mathbb{R}^{n}\right)$, $f$ denota o operador de multiplicação ponto a ponto por $f, e[$,$] é um comutador.$

Demonstração: Por definição:

$$
\left[\left(I+t^{2} L\right)^{-1}, f\right] u(x)=\left(I+t^{2} L\right)^{-1} u(x) f(x)-f(x)\left(I+t^{2} L\right)^{-1} u(x)
$$

Pode-se re-escrever isto como sendo:

$$
\begin{aligned}
& {\left[\left(I+t^{2} L\right)^{-1}, f\right] u(x)} \\
& =\left(I+t^{2} L\right)^{-1}(f u)(x)-f\left(I+t^{2} L\right)^{-1} u(x) \\
& =-f\left(I+t^{2} L\right)^{-1} u(x)+\left(I+t^{2} L\right)^{-1}(f u)(x) \\
& =-I f\left(I+t^{2} L\right)^{-1} u(x)+\left(I+t^{2} L\right)^{-1}(f I u)(x), \text { onde I é a identidade. } \\
& =-\left(I+t^{2} L\right)^{-1}\left(I+t^{2} L\right) f\left(I+t^{2} L\right)^{-1} u(x)+\left(I+t^{2} L\right)^{-1}\left(f\left(I+t^{2} L\right)\left(I+t^{2} L\right)^{-1} u\right)(x)
\end{aligned}
$$


Fatorando $\left(I+t^{2} L\right)^{-1}$ pela esquerda, obtemos:

$$
\begin{aligned}
= & -\left(I+t^{2} L\right)^{-1}\left(I+t^{2} L\right) f\left(I+t^{2} L\right)^{-1} u(x)+\left(I+t^{2} L\right)^{-1}\left(f\left(I+t^{2} L\right)\left(I+t^{2} L\right)^{-1} u\right)(x) \\
= & -\left(I+t^{2} L\right)\left[\left(I+t^{2} L\right) f\left(I+t^{2} L\right)^{-1} u(x)-f\left(I+t^{2} L\right)\left(I+t^{2} L\right)^{-1} u(x)\right] \\
= & -\left(I+t^{2} L\right)\left[f\left(I+t^{2} L\right)^{-1} u(x)+t^{2} L f\left(I+t^{2} L\right)^{-1} u(x)\right. \\
& \left.-f\left(I+t^{2} L\right)^{-1} u(x)-t^{2} f L\left(I+t^{2} L\right)^{-1} u(x)\right] \\
= & -\left(I+t^{2} L\right)\left[t^{2} L f\left(I+t^{2} L\right)^{-1} u(x)-t^{2} f L\left(I+t^{2} L\right)^{-1} u(x)\right] \\
= & -\left(I+t^{2} L\right) t^{2}[L, f]\left(I+t^{2} L\right)^{-1} u(x) .
\end{aligned}
$$

Onde a ultima igualdade é dada pela definição de comutador para $\left(I+t^{2} L\right)^{-1} u$.

Afirmação: $[L, f] u(x)=-\nabla f \cdot A \nabla u(x)-\operatorname{div} A(\nabla f \cdot u)(x)$

De fato, usando a definição de nosso operador $L$, podemos notar que:

$$
\begin{aligned}
{[L, f] u(x) } & =L(u f)(x)-f L u(x) \\
& =-\operatorname{div} A \nabla(f \cdot u)(x)+f \cdot \operatorname{div} A \nabla u(x) \\
& =-\operatorname{div} A(f \nabla u)(x)-\operatorname{div} A(u \nabla f)(x)+f \cdot \operatorname{div} A \nabla u(x) \\
& =-f \cdot \operatorname{div} A \nabla u(x)-\nabla f \cdot A \nabla u(x)-\operatorname{div} A(u \nabla f)(x)+f \cdot \operatorname{div} A \nabla u(x) \\
& =-\nabla f \cdot A \nabla u(x)-\operatorname{div} A(\nabla f \cdot u)(x) .
\end{aligned}
$$

Então usando a afirmação para $\left(I+t^{2} L\right)^{-1} u(x)$

$$
\begin{aligned}
& {\left[\left(I+t^{2} L\right)^{-1}, f\right] u(x)} \\
& =-\left(I+t^{2} L\right)^{-1} t^{2}\left(-\nabla f \cdot A \nabla\left(I+t^{2} L\right)^{-1} u(x)-\operatorname{div} A\left(\nabla f \cdot\left(I+t^{2} L\right)^{-1} u\right)(x)\right) \\
& =t\left(I+t^{2} L\right)^{-1} \nabla f \cdot A \cdot t \nabla\left(I+t^{2} L\right)^{-1} u(x)+t\left(I+t^{2} L\right)^{-1} t \operatorname{div} A\left(\nabla f \cdot\left(I+t^{2} L\right)^{-1} u\right)(x)
\end{aligned}
$$

Aqui, no primeiro termo da soma, $\nabla f \cdot A \cdot t \nabla\left(I+t^{2} L\right)^{-1} u$, é um elemento do espaço $L^{2}$ e no segundo termo, $A\left(\nabla f \cdot\left(I+t^{2} L\right)^{-1} u\right)$, também. Por tanto, das propriedades $L^{2}$-limitantes de $\left(I+t^{2} L\right)^{-1}, t \nabla\left(I+t^{2} L\right)^{-1}$ e $\left(I+t^{2} L\right)^{-1} t$ div, fazemos:

$$
\begin{aligned}
& \left\|\left[\left(I+t^{2} L\right)^{-1}, f\right] u\right\|_{L^{2}} \\
& \leq t\left\|\left(I+t^{2} L\right)^{-1} \nabla f \cdot A \cdot t \nabla\left(I+t^{2} L\right)^{-1} u\right\|_{L^{2}}+t\left\|\left(I+t^{2} L\right)^{-1} t \operatorname{div} A \nabla f \cdot\left(I+t^{2} L\right)^{-1} u\right\|_{L^{2}} \\
& \leq C_{1} t\left\|\nabla f \cdot A \cdot t \nabla\left(I+t^{2} L\right)^{-1} u\right\|_{L^{2}}+C_{2} t\left\|A \nabla f \cdot\left(I+t^{2} L\right)^{-1} u\right\|_{L^{2}} \\
& \leq C_{1} t\left\|A^{T} \nabla f\right\| \cdot\left\|t \nabla\left(I+t^{2} L\right)^{-1} u\right\|_{L^{2}}+C_{2} t\|A \nabla f\| \cdot\left\|\left(I+t^{2} L\right)^{-1} u\right\|_{L^{2}} \\
& \leq C_{1} t\left\|A^{T} \nabla f\right\| \cdot\|u\|_{L^{2}}+C_{2} t\|A \nabla f\| \cdot\|u\|_{L^{2}}
\end{aligned}
$$


Pela definição da matriz $A$ e como $f$ é Lipschitz, então as normas $\left\|A^{T} \nabla f\right\|$ e $\|A \nabla f\|$ são limitadas por $C_{3}\|\nabla f\|_{\infty}$, obtendo assim:

$$
\begin{gathered}
\left\|\left[\left(I+t^{2} L\right)^{-1}, f\right] u\right\|_{L^{2}} \leq C t\|\nabla f\|_{\infty} \cdot\|u\|_{L^{2}} \\
\left\|\left[\left(I+t^{2} L\right)^{-1}, f\right]\right\|_{o p}=\sup _{\|u\| \leq 1}\left\|\left[\left(I+t^{2} L\right)^{-1}, f\right] u\right\|_{L^{2}} \leq C t\|\nabla f\|_{\infty}
\end{gathered}
$$

onde $C$ depende apenas de $\lambda, \Lambda$ e $n$.

Para a segunda desigualdade, fazermos uso da propriedade $L^{2}$-limitante do operador $t^{2} \nabla\left(I+t^{2} L\right)^{-1}$ div. Dessa maneira, aplicando o gradiente em $\left(^{*}\right)$, segue que:

$$
\begin{aligned}
& \nabla\left[\left(I+t^{2} L\right)^{-1}, f\right] u(x) \\
& =\nabla t\left(I+t^{2} L\right)^{-1} \nabla f \cdot A \cdot t \nabla\left(I+t^{2} L\right)^{-1} u(x)+\nabla t\left(I+t^{2} L\right)^{-1} t \operatorname{div} A\left(\nabla f \cdot\left(I+t^{2} L\right)^{-1} u\right)(x) \\
& =t \nabla\left(I+t^{2} L\right)^{-1} \nabla f \cdot A \cdot t \nabla\left(I+t^{2} L\right)^{-1} u(x)+t^{2} \nabla\left(I+t^{2} L\right)^{-1} \operatorname{div} A\left(\nabla f \cdot\left(I+t^{2} L\right)^{-1} u\right)(x) \\
& \Rightarrow \\
& \left\|\nabla\left[\left(I+t^{2} L\right)^{-1}, f\right] u\right\|_{L^{2}} \\
& \leq\left\|t \nabla\left(I+t^{2} L\right)^{-1} \nabla f \cdot A \cdot t \nabla\left(I+t^{2} L\right)^{-1} u\right\|+\left\|t^{2} \nabla\left(I+t^{2} L\right)^{-1} \operatorname{div} A\left(\nabla f \cdot\left(I+t^{2} L\right)^{-1} u\right)\right\| \\
& \leq C_{1}\left\|\nabla f \cdot A \cdot t \nabla\left(I+t^{2} L\right)^{-1} u\right\|+C_{2}\left\|A \nabla f \cdot\left(I+t^{2} L\right)^{-1} u\right\| \\
& \leq C\|\nabla f\|_{\infty} \cdot\|u\|_{L^{2}} \\
& \Rightarrow \quad\left\|\nabla\left[\left(I+t^{2} L\right)^{-1}, f\right]\right\|_{o p}=\sup _{\|u\| \leq 1}\left\|\nabla\left[\left(I+t^{2} L\right)^{-1}, f\right] u\right\|_{L^{2}} \leq C\|\nabla f\|_{\infty}
\end{aligned}
$$

onde $C$ depende apenas de $\lambda, \Lambda$ e $n$.

Por cubo em $\mathbb{R}^{n}$, entenda-se por um cubo com lados paralelos aos eixos. Se $Q$ é um cubo, então $|Q|$ e $\ell(Q)$ denotarão respetivamente sua medida, e seu comprimento de lado. Usaremos também a notação $c Q$ para denotar o cubo concêntrico com $Q$ que tem comprimento de lado igual a $c \ell(Q)$.

Lema 2.3 Para alguma constante $C$ dependendo somente de $n, \lambda$ e $\Lambda$; se $Q$ é um cubo em $\mathbb{R}^{n}, t \leq|Q|$ e f é uma função Lipschitz em $\mathbb{R}^{n}$, têm-se que:

$$
\begin{gathered}
\int_{Q}\left|\left(I+t^{2} L\right)^{-1} f-f\right|^{2} d x \leq C t^{2}\|\nabla f\|_{\infty}^{2}|Q| \\
\int_{Q}\left|\nabla\left(\left(I+t^{2} L\right)^{-1} f-f\right)\right|^{2} d x \leq C\|\nabla f\|_{\infty}^{2}|Q| .
\end{gathered}
$$




\section{CAPÍTULO 2. ESTIMATIVAS DE OPERADORES ELÍPTICOS EM $\mathbb{R}^{N}$}

Demonstração: Para provar o lema 2.3, precisarmos do seguinte teorema.

Teorema 2.4 (Propriedade da conservação do resolvente). Seja L o operador definido em (2.2). A seguinte identidade é valida:

$$
\left(I+t^{2} L\right)^{-1}(1)=1
$$

no sentido que $\left(I+t^{2} L\right)^{-1}\left(\eta_{R}\right) \longrightarrow 1$ em $L_{\text {loc }}^{2}\left(\mathbb{R}^{n}\right)$, quando $R \longrightarrow \infty$, onde $\eta_{R}=\eta(\dot{\bar{R}})$ é uma função com a propriedade: para cada $N>0$

$$
\int_{Q_{N}}\left|\left(I+t^{2} L\right)^{-1} \eta_{R}-1\right|^{2} d x \longrightarrow 0, \text { quando } R \rightarrow \infty
$$

onde $Q_{N}$ é um cubo centrado no origem com comprimento de lado $2 N$.

Prova: Fixa-se $\eta$ como antes, e seja $\eta_{R}(x)=\eta\left(\frac{x}{R}\right)$. Então, para qualquer $N>0$, considere um $R$ suficientemente grande de maneira que $\eta_{R} \equiv 1$, em $Q_{N}$. Assim:

$$
\begin{aligned}
\int_{Q_{N}}\left|\left(I+t^{2} L\right)^{-1} \eta_{R}-1\right|^{2} d x & =\int_{Q_{N}}\left|\left(I+t^{2} L\right)^{-1} \eta_{R}-\eta_{R}\right|^{2} d x \\
& =\int_{Q_{N}}\left|\left(I+t^{2} L\right)^{-1}\left[\eta_{R}-\left(I+t^{2} L\right) \eta_{R}\right]\right|^{2} d x \\
& =\int_{Q_{N}}\left|\left(I+t^{2} L\right)^{-1}\left[t^{2} L \eta_{R}\right]\right|^{2} d x
\end{aligned}
$$

Seja $H_{R}:=\operatorname{supp}\left(\nabla \eta_{R}\right)$ e $d=d(N, R)=\operatorname{dist}\left(Q_{N}, H_{R}\right)$, então afirmamos que $d \longrightarrow \infty$, quando $R \longrightarrow \infty$. De fato, assuma que $J \subset \mathbb{R}^{n}$ é um conjunto aberto, tal que $J$ contenha o origem e $\eta(x)=1, \forall x \in J$. Assuma também, sem perda de generalidade, que para um $R$ suficientemente grande, $\frac{x}{R}<x$; o que implica que $\frac{x}{R}$ está mais perto do origem e portanto, $\eta\left(\frac{x}{R}\right)=1, \forall x \in J$. Mas , existem $x \in \mathbb{R}^{n}$ fora de $J$ que satisfazem $\eta\left(\frac{x}{R}\right)=1$. Esses $x$ estão em um conjunto que denotaremos por $R J:=\{R \cdot x, x \in J\}$ e assim, a função $\eta_{R}$ tem a propriedade de ser igual à 1 em $R J$, esto é, $\nabla \eta_{R} \equiv 0, \forall x \in J$. Como $H_{R}:=\operatorname{supp}\left(\nabla \eta_{R}\right)$, segue que para um $\mathrm{R}$ suficientemente grande, o conjunto $R J$ contém propriamente a $Q_{N}$. Além disso, $H_{R} \subset J R^{c}$, por tanto, $d(N, R)>0$ e esse valor vai crescendo quando $R$ vai se aproximando para o infinito. Resumindo, temos dois conjuntos fechados, $H_{R}$ e $Q_{N}$, distintos do vazio, cuja distância entre eles é maior do que 0. Então, podemos aplicar a terceira desigualdade do lema 2.1 para $E=H_{R}$ e 
$F=Q_{N}$ e $\mathbf{f}=A \nabla \eta_{R}$

$$
\begin{aligned}
\int_{Q_{N}}\left|\left(I+t^{2} L\right)^{-1} \eta_{R}-1\right|^{2} d x & =\int_{Q_{N}}\left|\left(I+t^{2} L\right)^{-1} t^{2} \operatorname{div}\left(A \nabla \eta_{R}\right)\right|^{2} d x \\
& =t^{2} \int_{Q_{N}}\left|\left(I+t^{2} L\right)^{-1} t \operatorname{div}\left(A \nabla \eta_{R}\right)\right|^{2} d x \\
& \leq t^{2} C e^{-\frac{d}{c t}} \int_{H_{R}}\left|\left(A \nabla \eta_{R}(x)\right)\right|^{2} d x \\
& =t^{2} C e^{-\frac{d}{c t}} \int_{H_{R}}|(A \nabla \eta(x / R))|^{2} d x \\
& \leq t^{2} C R^{-1} e^{-\frac{d}{c t}}\|\nabla \eta\|_{\infty} \int_{H_{R}} d x \\
& \leq t^{2} C R^{-1} e^{-\frac{d}{c t}}\|\nabla \eta\|_{\infty}\left|H_{R}\right|
\end{aligned}
$$

Como $t \leq \ell(Q)$ e $d$ vai para $\infty$ junto com $R$, segue q último termo converge a 0 , quando $R \longrightarrow \infty$. Assim:

$$
\lim _{R \rightarrow+\infty} \int_{Q_{N}}\left|\left(I+t^{2} L\right)^{-1} \eta_{R}-1\right|^{2} d x=0
$$

$\Rightarrow$

$$
\lim _{R \rightarrow+\infty}\left(I+t^{2} L\right)^{-1} \eta_{R}=1
$$

em $L_{l o c}^{2}\left(\mathbb{R}^{n}\right)$. Isso finaliza a prova do teorema 2.4 .

Continuando com a demonstração do lema 2.3. Considere $\left\{\mathcal{X}_{k}\right\}$ uma partição da unidade, isto é, uma família de funções tal que $\mathcal{X}_{k}: \mathbb{R}^{n} \longrightarrow[0,1]$ e para cada $x \in \mathbb{R}^{n}$ :

- Existe uma vizinhança $V$ de $x$, tal que $\mathcal{X}_{k}(x) \neq 0$ em $V$, para uma quantidade finita de funções $\mathcal{X}_{k}$.

- A soma de todas essas funções em $x$ é igual a 1, i.e:

$$
\sum_{k \in I}=\mathcal{X}_{k}(x)=1
$$

Por redimensionamento, não há nenhuma perda de generalidade assumir que $\ell(Q)=1$ e que $\|\nabla f\|_{\infty}=1$. Tome-se uma partição $\left\{Q_{k}\right\}$ de $\mathbb{R}^{n}$ por cubos de comprimento de lado igual a 2 , com $Q_{0}=2 Q$. Seja $\mathcal{X}_{k}$ (os mesmos definidos 
anteriormente) a função indicadora de $Q_{k}$. Pelo teorema anterior, sabemos que $\left(I+t^{2} L\right)^{-1} 1=1$. Assim, podemos escrever:

$$
\begin{aligned}
\left(I+t^{2} L\right)^{-1} f(x)-f(x) & =\sum_{k \in \mathbb{Z}^{n}}\left[\left(I+t^{2} L\right)^{-1}\left(f \cdot \mathcal{X}_{k}\right)(x)\right]-f(x) \\
& =\sum_{k \in \mathbb{Z}^{n}}\left[\left(I+t^{2} L\right)^{-1}\left(f \cdot \mathcal{X}_{k}\right)(x)\right]-1 . f(x) \\
& =\sum_{k \in \mathbb{Z}^{n}}\left[\left(I+t^{2} L\right)^{-1}\left(f \cdot \mathcal{X}_{k}\right)(x)\right]-\left(I+t^{2} L\right)^{-1} 1 . f(x) \\
& =\sum_{k \in \mathbb{Z}^{n}}\left[\left(I+t^{2} L\right)^{-1}\left(f \cdot \mathcal{X}_{k}\right)(x)\right]-\sum_{k \in \mathbb{Z}^{n}}\left(I+t^{2} L\right)^{-1} \mathcal{X}_{k} . f(x) \\
& =\sum_{k \in \mathbb{Z}^{n}}\left[\left(I+t^{2} L\right)^{-1}\left(f \cdot \mathcal{X}_{k}\right)-\left(I+t^{2} L\right)^{-1} f(x) . \mathcal{X}_{k}\right](x) \\
& =\sum_{k \in \mathbb{Z}^{n}}\left[\left(I+t^{2} L\right)^{-1}(f-f(x)) \mathcal{X}_{k}\right](x)=: \sum_{k \in \mathbb{Z}^{n}} g_{k}(x) .
\end{aligned}
$$

Para $k=0$, temos que:

$$
\begin{aligned}
{\left[\left(I+t^{2} L\right)^{-1}(f-f(x)) \mathcal{X}_{k}\right](x) } & =\left(I+t^{2} L\right)^{-1}\left(f \cdot \mathcal{X}_{0}-f(x) . \mathcal{X}_{0}\right)(x) \\
& \left.=\left(I+t^{2} L\right)^{-1}\left(f \cdot \mathcal{X}_{0}\right)(x)-f(x) \cdot\left(I+t^{2} L\right)^{-1} \mathcal{X}_{0}\right)(x) \\
& =\left[\left(I+t^{2} L\right)^{-1}, f\right] \mathcal{X}_{0}(x) .
\end{aligned}
$$

o qual, pela primeira estimativa do lema 2.2, é limitado por $C t\left\|\mathcal{X}_{0}\right\|$.

Os termos $k \neq 0$ são tratados usando uma decomposição adicional:

$$
\begin{aligned}
& g_{k}(x)=\left(I+t^{2} L\right)^{-1}\left((f-f(x)) \mathcal{X}_{k}\right)(x) \pm\left(I+t^{2} L\right)^{-1}\left(f\left(x_{k}\right) \cdot \mathcal{X}_{k}\right)(x) \\
& g_{k}(x)=\left(I+t^{2} L\right)^{-1}\left(\left(f-f\left(x_{k}\right)\right) \mathcal{X}_{k}\right)(x)+\left(f\left(x_{k}\right)-f(x)\right)\left(I+t^{2} L\right)^{-1} \mathcal{X}_{k}(x)
\end{aligned}
$$

onde $x_{k}$ é o centro de $Q_{k}$. Usando o lema 2.1 para o operador $\left(I+t^{2} L\right)^{-1}$ sobre os conjuntos $E=Q_{k}$ e $F=Q$ (conjuntos disjuntos pois $Q_{0}$ contém propriamente a $Q)$ e o fato de que $f$ é Lipschitz, temos que:

$$
\int_{Q}\left|g_{k}(x)\right|^{2} d x \leq C t^{2}|Q|
$$

Com o qual mostramos a primeira estimativa.(Veja [7] para mais detalhe). Da mesma maneira que foi feita no Lema 2.2., pode-se obter a segunda estimativa. Finalmente, concluímos com um corolário como resultado dos lemas anteriores.

Corolário 2.5 Suponha que $\mathbf{f} \in\left(L^{\infty}\left(\mathbb{R}^{n}\right)\right)^{n}$. Então para cada $y \in \mathbb{R}^{n}$ e $t>0$ :

$$
\frac{1}{\left|B_{t}(y)\right|} \int_{B_{4 t}(y)}\left|t\left(I+t^{2} L\right)^{-1} \operatorname{div} A \mathbf{f}(x)\right|^{2} d x \leq C\|\mathbf{f}\|_{\infty}^{2} .
$$

Prova: Ver [7] ou [12]. 


\section{Capítulo 3}

\section{Redução à estimativa de função quadrática}

\subsection{Representação do operador raiz quadrada}

Demos um operador elíptico definido em (2.2) com constantes de elipticidade dadas em (2.1). Desejamos provar que:

$$
\|\sqrt{L} f\|_{L^{2}} \leq C\|\nabla f\|_{L^{2}}
$$

para $f$ em algum subespaço denso de $H^{1}\left(\mathbb{R}^{n}\right)$ com $C$ dependendo somente de $n, \lambda$ e $\Lambda$. Então, a estimativa $(\mathrm{K})$ também é valida para $L^{*}$ sobre certas hipóteses de operadores adjuntos. Finalmente, concluirmos com um teorema de J.L.Lions ([27]) que o domínio de $\sqrt{L}$ é $H^{1}\left(\mathbb{R}^{n}\right)$ e que para cada $f \in H^{1}\left(\mathbb{R}^{n}\right)$, temos:

$$
\|\sqrt{L} f\|_{L^{2}} \sim\|\nabla f\|_{L^{2}}
$$

- Observamos que para provar $(\mathrm{K})$, podemos assumir temporariamente que os coeficientes são $C^{\infty}$, desde que não usemos isso quantitativamente em nossas estimativas. É por isso que vamos deixar claro a dependência das constantes. Logo, remove-se essa suposição usando uma ligeira variante de ([10], Cap 0,prop 7). Para começar, usamos a seguinte resolução da raiz quadrada dada pelo teorema 1.29, onde escrevermos a potência fracionária desejada, como suma de dois exponentes, $\operatorname{com} z=\frac{3}{2}$ e $n=3$ 
38CAPÍTULO 3. REDUÇÃO À ESTIMATIVA DE FUNÇÃO QUADRÁTICA

$$
\begin{aligned}
\sqrt{L}=L^{1 / 2}=L^{-1}\left(L^{3 / 2} f\right) & =L^{-1} a \int_{0}^{\infty} t^{\left(\frac{3}{2}-1\right)} A^{3} R(t:-A)^{3} f d t \\
& =L^{-1} a \int_{0}^{\infty} t^{1 / 2} L^{3} R(t:-L)^{3} f d t \\
& =a \int_{0}^{\infty} t^{1 / 2} L^{2} R(t:-L)^{3} f d t \\
& =a \int_{0}^{\infty} t^{1 / 2} L^{2}(t I+L)^{-3} f d t .
\end{aligned}
$$

onde $a$ é um valor numérico que detalharemos mais adiante. Fazendo uma mudança de variáveis $t \longmapsto t^{-2}$, temos:

$$
\begin{aligned}
\sqrt{L} & =-a \int_{\infty}^{0} t^{-1} L^{2}\left(t^{-2} I+L\right)^{-3} f t^{-3} d t \\
& =a \int_{0}^{\infty} t^{-4} L^{2}\left(t^{-2} I+L\right)^{-3} f d t \\
& =a \int_{0}^{\infty} t^{2} t^{-6}\left(t^{-2} I+L\right)^{-3} L^{2} f d t \\
& =a \int_{0}^{\infty}\left[t^{-2}\left(t^{-2} I+L\right)^{-1}\right]\left[t^{-2}\left(t^{-2} I+L\right)^{-1}\right]\left[t^{-2}\left(t^{-2} I+L\right)^{-1}\right] t^{2} L^{2} f d t \\
& =a \int_{0}^{\infty}\left[\left(I+t^{2} L\right)^{-1}\right]\left[\left(I+t^{2} L\right)^{-1}\right]\left[\left(I+t^{2} L\right)^{-1}\right] t^{2} L^{2} f d t \\
& =a \int_{0}^{\infty}\left[\left(I+t^{2} L\right)^{-3}\right] t^{2} L^{2} f d t \\
& =a \int_{0}^{\infty}\left(I+t^{2} L\right)^{-3} t^{3} L^{2} f \frac{d t}{t} .
\end{aligned}
$$

onde usamos a afirmação do corolário 1.14 para modificar o resolvente. o valor de $a$ é dado por $L=I$ e $f=1$, para obter:

$$
a^{-1}=\int_{0}^{\infty}\left(1+u^{2}\right)^{-3} u^{2} d u
$$


. Então, a anterior integral converge para $f \in \mathcal{D}\left(L^{2}\right)$,pelo teorema 1.29. Considere uma função $g \in C_{0}^{\infty}\left(\mathbb{R}^{n}\right)$ com $\|g\|_{L^{2}}=1$. Observe que:

$$
\begin{aligned}
& |\langle\sqrt{L}, g\rangle|^{2}=\left|\left\langle a \int_{0}^{\infty}\left(I+t^{2} L\right)^{-3} t^{3} L^{2} f \frac{d t}{t}, g\right\rangle\right|^{2} \\
& =\left|a \int_{\mathbb{R}^{n}} \int_{0}^{\infty}\left(I+t^{2} L\right)^{-3} t^{3} L^{2} f(x) \cdot \overline{g(x)} \frac{d t}{t} d x\right|^{2} \\
& =\left|a \int_{0}^{\infty} \int_{\mathbb{R}^{n}} t^{2} L\left(I+t^{2} L\right)^{-2}\left(I+t^{2} L\right)^{-1} t L f(x) \cdot \overline{g(x)} d x \frac{d t}{t}\right|^{2} \\
& =\left|a \int_{0}^{\infty}\left\langle t^{2} L\left(I+t^{2} L\right)^{-2}\left(I+t^{2} L\right)^{-1} t L f, \bar{g}\right\rangle \frac{d t}{t}\right|^{2} \\
& =\left|a \int_{0}^{\infty}\left\langle\left(I+t^{2} L\right)^{-1} t L f,\left(\left(I+t^{2} L\right)^{-2}\right)^{*} t^{2} L^{*} \bar{g}\right\rangle \frac{d t}{t}\right|^{2} \\
& =\left|a \int_{0}^{\infty}\left\langle\left(I+t^{2} L\right)^{-1} t L f,\left(1+t^{2} L^{*}\right)^{-2} t^{2} L^{*} \bar{g}\right\rangle \frac{d t}{t}\right|^{2} \\
& \leq\left|a \int_{0}^{\infty}\right|\left\langle\left(I+t^{2} L\right)^{-1} t L f,\left(1+t^{2} L^{*}\right)^{-2} t^{2} L^{*} \bar{g}\right\rangle\left|\frac{d t}{t}\right|^{2} \\
& \leq a^{2}\left|\int_{0}^{\infty}\left\|\left(I+t^{2} L\right)^{-1} t L f\right\|_{L^{2}}\left\|\left(1+t^{2} L^{*}\right)^{-2} t^{2} L^{*} \bar{g}\right\|_{L^{2}} \frac{d t}{t}\right|^{2} \\
& \leq a^{2} \int_{0}^{\infty}\left\|\left(I+t^{2} L\right)^{-1} t L f\right\|_{L^{2}}^{2} \frac{d t}{t} \int_{0}^{\infty}\left\|\left(1+t^{2} L^{*}\right)^{-2} t^{2} L^{*} \bar{g}\right\|_{L^{2}}^{2} \frac{d t}{t} \\
& =a^{2} \int_{0}^{\infty}\left\|\left(I+t^{2} L\right)^{-1} t L f\right\|_{L^{2}}^{2} \frac{d t}{t} \int_{0}^{\infty}\left\|V_{t} g\right\|_{L^{2}}^{2} \frac{d t}{t} .
\end{aligned}
$$

onde $V_{t} g=\left(1+t^{2} L^{*}\right)^{-2} t^{2} L^{*} \bar{g}$. Nesta seção vamos provar a limitação uniforme da integral que tem como argumento $\left\|V_{t} g\right\|_{L^{2}}^{2}$,ou seja:

$$
\int_{0}^{\infty}\left\|V_{t} g\right\|_{L^{2}}^{2} \frac{d t}{t} \leq C\|g\|_{L^{2}}
$$

Com isso, provar $(\mathrm{K})$ se reduz a mostrar

$$
\int_{0}^{\infty}\left\|\left(I+t^{2} L\right)^{-1} t L f\right\|_{L^{2}}^{2} \frac{d t}{t} \leq C\|\nabla f\|_{L^{2}}^{2} .
$$


Para provar (3.1), vamos nôs basar em [20] Apêndice 7.3.1.

Considere $V_{t} g$ como antes e veja-se que:

$$
\begin{aligned}
\int_{0}^{\infty}\left\|V_{t} g\right\|_{L^{2}}^{2} \frac{d t}{t} & =\sum_{k=-\infty}^{\infty} \int_{2^{k}}^{2^{k+1}}\left\|V_{t} g\right\|_{L^{2}}^{2} \frac{d t}{t} \\
& =\sum_{k=-\infty}^{\infty} \int_{2^{k}}^{2^{k+1}} \int_{\mathbb{R}^{n}}\left|\left(1+t^{2} L^{*}\right)^{-2} t^{2} L^{*} g(x)\right|^{2} d x \frac{d t}{t} \\
& =\sum_{k=-\infty}^{\infty} \int_{1}^{2} \int_{\mathbb{R}^{n}}\left|\left(1+2^{2 k} t^{2} L^{*}\right)^{-2} 2^{2 k} t^{2} L^{*} g(x)\right|^{2} d x \frac{d t}{t} \\
& =\sum_{k=-\infty}^{\infty} \int_{1}^{2} \int_{\mathbb{R}^{n}}\left|\left(1+\left(2^{k} t\right)^{2} L^{*}\right)^{-2}\left(2^{k} t\right)^{2} L^{*} g(x)\right|^{2} d x \frac{d t}{t} \\
& =\sum_{k=-\infty}^{\infty} \int_{1}^{2}\left\|V_{2^{k} t} g\right\|_{L^{2}}^{2} \frac{d t}{t}
\end{aligned}
$$

Onde usamos uma mudança de variáveis para $t \rightarrow 2^{k} t$. Denote por $\left\{r_{k}\right\}_{k \in \mathbb{Z}}$ a base ortonormal de Rademacher em $L^{2}([0,1])$, onde $r_{k}(x) \in\{-1,1\}$ e $r_{k}$ é constante sobre subconjuntos diádicos de $[0,1]$ suficientemente pequenos sobre $k$. Então, já que $\int_{0}^{1} r_{k}(s) r_{j}(s) d s=\delta_{k j}$, para cada $t>0$ e para cada inteiro positivo $N$ :

$$
\begin{aligned}
\sum_{k=-N}^{N}\left\|V_{2^{k}} g\right\|_{L^{2}}^{2} & =\int_{\mathbb{R}^{n}} \sum_{k=-N}^{N}\left|V_{2^{k} t} g\right|^{2} d x \\
& =\int_{\mathbb{R}^{n}} \sum_{k=-N}^{N} \sum_{j=-N}^{N} \delta_{k j}\left(V_{2^{k} t} g\right)\left(V_{2^{j} t} g\right) d x \\
& =\int_{\mathbb{R}^{n}} \sum_{k=-N}^{N} \sum_{j=-N}^{N} \int_{0}^{1} r_{k}(s) r_{j}(s)\left(V_{2^{k} t} g\right)\left(V_{2^{j}} g\right) d s d x \\
& =\int_{0}^{1} \sum_{k=-N}^{N} \sum_{j=-N}^{N} \int_{\mathbb{R}^{n}} r_{k}(s) r_{j}(s)\left(V_{2^{k} t} g\right)\left(V_{2^{j} t} g\right) d x d s \\
& =\int_{0}^{1}\left\|\sum_{k=-N}^{N} r_{k}(s)\left(V_{2^{k} t} g\right)\right\|_{L^{2}}^{2} d s
\end{aligned}
$$

No sentido do análise funcional e o calculo funcional natural, o operador $V_{t}$ é dado por:

$$
V_{t} f=\phi\left(t^{2} L^{*}\right) f, \quad \text { onde } \phi(z)=(1+z)^{-2} z
$$


Então:

$$
\begin{aligned}
\sum_{k=-N}^{N}\left\|V_{2^{k}} g\right\|_{L^{2}}^{2} & =\int_{0}^{1}\left\|\sum_{k=-N}^{N} r_{k}(s) \phi\left(2^{2 k} t^{2} L^{*}\right)\right\|_{L^{2}}^{2} d s \\
& \leq C\left(\int_{0}^{1} \sup _{z \in S_{\varphi}}\left|\sum_{k=-N}^{N} r_{k}(s) \phi\left(2^{2 k} t^{2} z\right)\right|^{2} d s\right)\|g\|_{L^{2}}^{2} \\
& \leq C\left(\sup _{z \in S_{\varphi}} \sum_{k \in \mathbb{Z}}\left|\phi\left(2^{2 k} t^{2} z\right)\right|^{2}\right)\|g\|_{L^{2}}^{2}
\end{aligned}
$$

Aqui foi usado o fato de que, como $L^{*}$ tem um calculo funcional natural limitado (Veja Apendice B ou [20] ), $\left\|\eta\left(L^{*}\right)\right\|_{\mathcal{B}\left(L^{2}\right)} \leq C\|\eta\|_{L}^{\infty}\left(S_{\omega}\right)$, para toda função $\eta \in$ $H_{0}^{\infty}\left(S_{\omega}\right)$, onde $S_{\omega}$ é um setor de ângulo $2 \omega$. Além disso, tem-se que para $z \in S_{\omega}$, $|\varphi(z)| \leq \min \left\{|z|,|z|^{-1}\right\}$, e segue que:

$$
\sup _{1 \leq t \leq 2} \sup _{z \in S_{\varphi}} \sum_{k \in \mathbb{Z}}\left|\phi\left(2^{2 k} t^{2} z\right)\right|^{2} \leq \sup _{1 \leq t \leq 2} \sup _{z \in S_{\varphi}} \sum_{k \in \mathbb{Z}} \min \left\{4^{k} t^{2}|z|, 4^{-k} t^{-2}|z|^{-1}\right\}
$$

Dado que $t \geq 1$, podemos reduzir a estimativa anterior a analisar simplesmente:

$$
\sup _{z \in S_{\varphi}} \sum_{k \in \mathbb{Z}} \min \left\{4^{k}|z|, 4^{-k}|z|^{-1}\right\}
$$

Isto é, analisar os valores mínimos de cada $k \in \mathbb{Z}$, para cada $z \in S_{\varphi}$. A primeira observação à ter em quenta é que a estimativa anterior pode-se subdividir em duas situações: quando $|z|<1$ e $|z|>1$. Dentre esses dois casos, novamente subdividimos em duas somatórias: quando $k \leq-1$ e quando $k \geq 0$. No caso em que $|z|<1$ junto com a condição de $k$ ser menor ou igual a -1 , a seguinte desigualdade é obtida:

$$
\begin{aligned}
\sum_{k \leq-1} \min \left\{4^{k}|z|, 4^{-k}|z|^{-1}\right\} & =\sum_{k \geq 1} \min \left\{4^{-k}|z|, 4^{k}|z|^{-1}\right\} \\
& =\sum_{k \geq 1} \min \left\{\frac{|z|}{4^{k}}, \frac{4^{k}}{|z|}\right\} \\
& =\sum_{k \geq 1} \frac{|z|}{4^{k}} \leq \sum_{k \geq 1} \frac{1}{4^{k}} \\
& =\frac{1}{3}
\end{aligned}
$$

Para o caso em que $|z|>1$ junto com a condição de $k$ ser maior o igual a 1, o procedimento é análogo pode-se obter a mesma limitação. Resta obter a limitação da série para o caso em que $|z|<1$, para $k \geq 0$; e por conseguente, o 
ocaso análogo: $|z|>1$ e $k \leq 0$. Para isso, veja que dado $|z|<1$ arbitrário, existe um $k_{0} \geq 0$ tal que:

Então:

$$
\frac{1}{4^{k_{0}}}<|z|<\frac{1}{4^{k_{0}-1}}
$$

$$
\begin{aligned}
\sum_{k=0}^{\infty} \min \left\{4^{k}|z|, 4^{-k}|z|^{-1}\right\} & =\sum_{k=0}^{k_{0}-1} \min \left\{4^{k}|z|, 4^{-k}|z|^{-1}\right\}+\sum_{k=k_{0}}^{\infty} \min \left\{4^{k}|z|, 4^{-k}|z|^{-1}\right\} \\
& =\sum_{k=0}^{k_{0}-1} 4^{k}|z|+\sum_{k=k_{0}}^{\infty} \frac{1}{4^{k}|z|} \\
& \leq \frac{1}{4^{k_{0}-1}} \sum_{k=0}^{k_{0}-1} 4^{k}|z|+4^{k_{0}} \sum_{k=k_{0}}^{\infty} \frac{1}{4^{k}|z|} \\
& =\frac{1}{4^{k_{0}-1}}\left(\frac{1-4^{k_{0}}}{1-4}\right)+4^{k_{0}}\left(\frac{1 / 4^{k_{0}}}{1-(1 / 4)}\right) \\
& =\frac{4}{4^{k_{0}}}\left(\frac{4^{k_{0}}-1}{3}\right)+\frac{4}{3} \\
& \leq \frac{4}{3}+\frac{4}{3} \\
& =\frac{8}{3} .
\end{aligned}
$$

Com isso, temos que $\sum_{k \in \mathbb{Z}} \min \left\{4^{k}|z|, 4^{-k}|z|^{-1}\right\}$ é estimada por $\frac{9}{3}$, para qualquer z. Mas, sabemos que:

$$
\sum_{k \in \mathbb{Z}} \min \left\{4^{k}, 4^{-k}\right\}=\frac{5}{3}
$$

Logo:

$$
\sup _{z \in S_{\varphi}} \sum_{k \in \mathbb{Z}} \min \left\{4^{k}|z|, 4^{-k}|z|^{-1}\right\} \leq 2 \sum_{k \in \mathbb{Z}} \min \left\{4^{k}, 4^{-k}\right\}
$$

Assim, voltando a nosso problema inicial, segue que:

$$
\begin{aligned}
\sup _{1 \leq t \leq 2} \sup _{z \in S_{\varphi}} \sum_{k \in \mathbb{Z}}\left|\phi\left(2^{2 k} t^{2} z\right)\right|^{2} & \leq C \sup _{1 \leq t \leq 2} \sup _{z \in S_{\varphi}} \sum_{k \in \mathbb{Z}} \min \left\{4^{k} t^{2}|z|, 4^{-k} t^{-2}|z|^{-1}\right\} \\
& \leq 2 \sup _{1 \leq t \leq 2} \sum_{k \in \mathbb{Z}} \min \left\{4^{k} t^{2}, 4^{-k} t^{-2}\right\} \\
& \leq \frac{16}{3}
\end{aligned}
$$

Portanto:

$$
\begin{aligned}
\int_{0}^{\infty}\left\|V_{t} g\right\|_{L^{2}}^{2} \frac{d t}{t} & =\sum_{k=-\infty}^{\infty} \int_{1}^{2}\left\|V_{2^{k} t} g\right\|_{L^{2}}^{2} \frac{d t}{t}=\lim _{N \rightarrow \infty} \int_{1}^{2} \sum_{k=-N}^{N}\left\|V_{2^{k} t} g\right\|_{L^{2}}^{2} \frac{d t}{t} \\
& \leq \lim _{N \rightarrow \infty} \sup _{1 \leq t \leq 2} \sum_{k=-N}^{N}\left\|V_{2^{k}} g\right\|_{L^{2}}^{2} \int_{1}^{2} \frac{d t}{t} \leq \lim _{N \rightarrow \infty} C\|g\|_{L^{2}}=C\|g\|_{L^{2}}
\end{aligned}
$$




\section{Capítulo 4}

\section{Redução à estimativa de medida Carleson}

No capítulo 2, demos um operador $L$ de tipo elíptico dado em (2.2) com constantes de elipticidade dadas em (2.1). Com base em isso, decidimos provar a conjetura enunciada por T.Kato, dada pela estimativa (K). Para provar tal estimativa, re-escrevemos o operador $=\sqrt{L}=L^{1 / 2}$ usando propriedades dadas por operadores com potências fracionárias positivas, para depois reduzir nossa estimativa a provar (3.2), previamente mostrando (3.1). Neste capítulo vamos reduzir, novamente, (3.2) a uma estimativa de medida Carleson.

\subsection{Redução à estimativa de função radial}

Vamos introduzir uma notação usada já anteriormente. Defina para funções de valor vetorial, isto é, com valores em $\mathbb{C}^{n}, \mathbf{f}=\left(f_{1}, f_{2}, \ldots, f_{n}\right) \in\left(L^{2}\left(\mathbb{R}^{n}\right)\right)^{n}$, a seguinte notação:

$$
\theta_{t} \mathbf{f}=-\left(I+t^{2} L\right)^{-1} t \operatorname{div} A \mathbf{f}
$$

(ou $\theta_{t} \mathbf{f}=-\left(I+t^{2} L\right)^{-1} t \frac{\partial}{\partial j}\left(a_{j k} f_{k}\right)$, onde usa-se o critério da somatória para índices repetidos). A primeira afirmação dada é que $\theta_{t} \in \mathcal{B}\left(\left(L^{2}\left(\mathbb{R}^{n}\right)\right)^{n}, L^{2}\left(\mathbb{R}^{n}\right)\right)$. De fato, pelas desigualdades obtidas no capítulo 1 , temos que, dado $g \in L^{2}\left(\mathbb{R}^{n}\right)$ com 
$\|g\|_{L_{2}}=1$ :

$$
\begin{aligned}
\left\langle\theta_{t} \mathbf{f}, g\right\rangle & =\int_{\mathbb{R}^{n}}-\left(I+t^{2} L\right)^{-1} t \operatorname{div} A \mathbf{f} \cdot \bar{g} d x \\
& =\int_{\mathbb{R}^{n}}-t \operatorname{div} A \mathbf{f} \cdot\left(1+t^{2} L^{*}\right)^{-1} \bar{g} d x \\
& =\int_{\mathbb{R}^{n}} A \mathbf{f} \cdot t \nabla\left(1+t^{2} L^{*}\right)^{-1} \bar{g} d x
\end{aligned}
$$

$\Rightarrow$

$$
\begin{aligned}
\left|\left\langle\theta_{t} \mathbf{f}, g\right\rangle\right| & \leq \Lambda \int_{\mathbb{R}^{n}}\left|\mathbf{f} \| t \nabla\left(1+t^{2} L^{*}\right)^{-1} \bar{g}\right| d x \\
& \leq \Lambda\|\mathbf{f}\|_{L^{2}}\left\|t \nabla\left(1+t^{2} L^{*}\right)^{-1} \bar{g}\right\|_{L^{2}} \\
& \leq C\|\mathbf{f}\|_{L^{2}} .
\end{aligned}
$$

Onde $C$ é uma constante que depende apenas de $n, \Lambda$ e $\lambda$. Com essa notação, (3.2) é rescrito como :

$$
\int_{\mathbb{R}^{n}}\left\|\theta_{t} \nabla f\right\|_{L^{2}}^{2} \frac{d t}{t} \leq C \int_{\mathbb{R}^{n}}|\nabla f|^{2}
$$

Para provar isso, precisamos introduzir primeiro um operador de suavização. Seja $P_{t} g(x)=\rho_{t} * g(x)$, onde $\rho_{t}(x)=t^{-n} \rho\left(\frac{x}{t}\right)$ satisfaz as seguintes condições:

- $\rho$ é radial.

- $\rho \in C_{0}^{\infty}\left(B_{1}(0)\right)$.

- $\int \rho d x=1$.

Agora, vamos afirmar que:

$$
\left(\widehat{\delta-P_{t}^{2}}\right) f(\xi)=t\left(\frac{\widehat{\rho}(t \xi)^{2}-1}{|t \xi|^{2}} i t \xi\right) \cdot \widehat{\nabla f}(\xi)=: \widehat{t R_{t} \nabla f}(\xi)
$$

Por uma parte temos:

$$
\begin{aligned}
\left(\widehat{\delta-P_{t}^{2}}\right) f(\xi) & =\widehat{\delta} f(\xi)-\widehat{P_{t}^{2}} f(\xi) \\
& \left.=\widehat{f}(\xi)-\widehat{P_{t}\left(P_{t}\right.} f\right)(\xi) \\
& =\widehat{f}(\xi)-\widehat{P_{t}\left(\rho_{t} * f\right)}(\xi) \\
& \left.=\widehat{f}(\xi)-\rho_{t} \widehat{*\left(\rho_{t} *\right.} f\right)(\xi) \\
& =\widehat{f}(\xi)-\widehat{\rho_{t}}(\xi) \cdot \widehat{\rho_{t}}(\xi) \cdot \widehat{f}(\xi) \\
& =\widehat{f}(\xi)-\frac{1}{t^{n}} \widehat{\rho}\left(\frac{\xi}{t}\right) \cdot \frac{1}{t^{n}} \widehat{\rho}\left(\frac{\xi}{t}\right) \cdot \widehat{f}(\xi) \\
& =\widehat{f}(\xi)-\widehat{\rho}(t \xi) \cdot \widehat{\rho}(t \xi) \cdot \widehat{f}(\xi) \\
& =\widehat{f}(\xi)-(\widehat{\rho}(t \xi))^{2} \cdot \widehat{f}(\xi) .
\end{aligned}
$$


E por outra tem-se:

$$
\begin{aligned}
t\left(\frac{\widehat{\rho}(t \xi)^{2}-1}{|t \xi|^{2}} i t \xi\right) \cdot \widehat{\nabla f}(\xi) & =\left(\frac{\widehat{\rho}(t \xi)^{2}-1}{|\xi|^{2}} i \xi\right) \cdot \widehat{\nabla f}(\xi) \\
& =\left(\frac{\widehat{\rho}(t \xi)^{2}-1}{|\xi|^{2}} i \xi\right) \cdot \widehat{\nabla f}(\xi) \\
& =\left(\frac{\widehat{\rho}(t \xi)^{2}-1}{|\xi|^{2}} i \xi\right) \cdot i \xi \widehat{f}(\xi) \\
& =\left(\frac{1-\widehat{\rho}(t \xi)^{2}}{|\xi|^{2}}\right) \cdot|\xi|^{2} \widehat{f}(\xi) \\
& =\left(1-\widehat{\rho}(t \xi)^{2}\right) \cdot \widehat{f}(\xi) \\
& =\widehat{f}(\xi)-(\widehat{\rho}(t \xi))^{2} \cdot \widehat{f}(\xi) .
\end{aligned}
$$

Uma vez provada a afirmação, vamos mostrar o quem é $R_{t} \nabla f$. Para isso, tome a seguinte igualdade

$$
t\left(\frac{\widehat{\rho}(t \xi)^{2}-1}{|t \xi|^{2}} i t \xi\right) \cdot \widehat{\nabla f}(\xi)=: t \widehat{R_{t} \nabla f}(\xi)
$$

$\Rightarrow$

$$
\left(\frac{\widehat{\rho}(t \xi)^{2}-1}{|t \xi|^{2}} i t \xi\right) \cdot \widehat{\nabla f}(\xi)=: \widehat{R_{t} \nabla f}(\xi)
$$

Observa-se que podemos escrever $\widehat{R_{t}} \mathbf{f}(\xi)=\widehat{r_{t} * \mathbf{f}}(\xi)=\widehat{r_{t}} \cdot \widehat{\mathbf{f}}(\xi)$. Se tomamos $\mathbf{f}=\nabla f$, a anterior identidade é escrita como:

$$
\widehat{R_{t} \nabla} f(\xi)=\widehat{r_{t}} \cdot \widehat{\mathbf{f}}(\xi)=\left(\frac{\widehat{\rho}(t \xi)^{2}-1}{|t \xi|^{2}} i t \xi\right) \cdot \widehat{\nabla f}(\xi), \forall \xi
$$

$\Rightarrow$

$$
\widehat{r}_{t}(\xi)=: \widehat{r}(\xi)=\frac{\widehat{\rho}(t \xi)^{2}-1}{|t \xi|^{2}} i t \xi .
$$

Algumas propriedades de funções em $L^{1}\left(\mathbb{R}^{n}\right)$ são:

1. $\widehat{f} \longrightarrow 0$, quando $|\xi| \rightarrow \infty$.

2. $\widehat{f} \in L^{\infty}$ e $\|\widehat{f}\|_{\infty} \leq\|f\|_{1}$.

3. $\widehat{f}$ é contínua em $\mathbb{R}^{n}$.

Além disso, $\widehat{r}(\xi)$ é holomorfa em um setor de tipo $S_{\pi}$. Veja-se também que $\widehat{r}(\xi)$ têm um decaimento em 0 e $\infty$. De fato, sendo $\rho$ a função que têm as propriedades anteriores:

$$
|\widehat{r}(\xi)|=\frac{\left|\widehat{\rho}(t \xi)^{2}-1\right|}{|t \xi|} \underset{|\xi| \rightarrow \infty}{\longrightarrow} 0 .
$$


As propriedades de $\rho$ mencionadas ao início implicam que $\widehat{\rho}(0)=1$ e $\widehat{\nabla \rho}(0)=0$. Assim

$$
\begin{aligned}
|\widehat{r}(\xi)| & =\frac{\left|\widehat{\rho}(t \xi)^{2}-1\right|}{|t \xi|} \\
& =\frac{|(\widehat{\rho}(t \xi)-1)(\widehat{\rho}(t \xi)+1)|}{|t \xi|} \\
& =\frac{\mid(\widehat{\rho}(t \xi)-\widehat{\rho}(0)-\widehat{\nabla \rho}(0) \mid}{|t \xi|}|\widehat{\rho}(t \xi)+1|
\end{aligned}
$$

Como $\widehat{\rho}$ é diferenciável em 0 , segue que o primeiro fator da última expressão é igual a $\widehat{\nabla \rho}(0)=0$, quando $|t \xi| \longrightarrow 0$. Assim $|\widehat{r}(\xi)| \longrightarrow 0$, quando $|\xi| \rightarrow 0$.Logo $\widehat{r}(\xi) \in H_{0}^{\infty}\left(S_{\pi}\right)$ e concluímos pelos resultados do cálculo funcional natural que:

$$
|\widehat{r}(\xi)| \leq C \min \left\{|\xi|,|\xi|^{-1}\right\}
$$

Agora considere um operador $Q_{s} f=\psi_{t} * f \operatorname{com} \psi \in C_{0}^{\infty}$ tal que $\widehat{\psi}(0)=0$ e $\int_{0}^{\infty} \widehat{\psi}_{t}(\xi)^{2} \frac{d t}{t}=1$. Então:

$$
\begin{aligned}
|\widehat{r}(t \xi)||\widehat{\psi}(s \xi)| & \leq C \min \left\{|t \xi|,|t \xi|^{-1}\right\} \cdot \min \left\{|s \xi|,|s \xi|^{-1}\right\} \\
& \leq C \min \left\{t, t^{-1}\right\} \cdot \min \left\{s, s^{-1}\right\} \\
& \leq C \min \left\{\frac{t}{s}, \frac{s}{t}\right\} .
\end{aligned}
$$

Dessa maneira, temos que dado $f \in L^{2}\left(\mathbb{R}^{n}\right)$, usando a identidade de Plancherel

$$
\begin{aligned}
\left\|R_{t} Q_{s} f\right\|_{L^{2}}^{2} & =\left\|r_{t} *\left(\psi_{s} * f\right)\right\|_{L^{2}}^{2} \\
& =\left\|\widehat{r_{t}} \cdot\left(\widehat{\psi_{s} * f}\right)\right\|_{L^{2}}^{2} \\
& =\left\|\widehat{r_{t}} \cdot \widehat{\psi_{s}} \cdot \widehat{f}\right\|_{L^{2}}^{2} \\
& =\int_{\mathbb{R}^{n}}\left|\widehat{r_{t}}(\xi) \cdot \widehat{\psi_{s}}(\xi) \cdot \widehat{f}(\xi)\right|^{2} d x \\
& =\int_{\mathbb{R}^{n}}|\widehat{r}(t \xi) \cdot \widehat{\psi}(s \xi) \cdot \widehat{f}(\xi)|^{2} d x \\
& \leq C \min \left\{\frac{t}{s}, \frac{s}{t}\right\}^{2} \int_{\mathbb{R}^{n}}|\widehat{f}(\xi)|^{2} d x \\
& =C \min \left\{\frac{t}{s}, \frac{s}{t}\right\}^{2}\|f\|_{L^{2}}^{2} \\
\Rightarrow \quad \| R_{t} Q_{s} & \|_{\mathcal{B}\left(L^{2}\right)} \leq C \min \left\{\frac{t}{s}, \frac{s}{t}\right\} .
\end{aligned}
$$


Por outra parte, já que $\rho \in C_{0}^{\infty}\left(B_{1}(0)\right)$ é radial, esta satisfaz a condição de momentos de fuga $\int x_{i} \rho d x=0$, para $i=1, \ldots, n$. Então, pela equação (35), capitulo 4, em [10], $r(x)$ satisfaz a limitação:

$$
|r(x)| \leq \frac{C}{|x|^{n-1}(1+|x|)^{2}} .
$$

Já que o lado direito da ultima desigualdade é integrável, segue do lema $2.1 \mathrm{em}$ [12] que $\left\|R_{t}\right\|_{\mathcal{B}\left(L^{2}\right)}$ é uniformemente limitada em $t$, com constantes dependendo somente de $n$ e a norma $L^{1}$ de $|r|$, e de maneira similar aplica-se a limitação para $\left\|R_{t} Q_{s}\right\|_{\mathcal{B}\left(L^{2}\right)}$. Então, aplicando o Corolário B.6, segue que para alguma constante $\beta>0$ :

$$
\left\|R_{t} Q_{s}\right\|_{\mathcal{B}\left(L^{2}\right)} \leq C \min \left\{\frac{t}{s}, \frac{s}{t}\right\}^{\beta} .
$$

Agora, já que pelo cálculo funcional natural tinha-se que:

$$
\left\|\theta_{t} \nabla\right\|_{\mathcal{B}\left(L^{2}\right)}=\left\|t\left(I+t^{2} L\right)^{-1} L\right\|_{\mathcal{B}\left(L^{2}\right)}=\frac{1}{t}\left\|t^{2}\left(I+t^{2} L\right)^{-1} L\right\|_{\mathcal{B}\left(L^{2}\right)} \leq C / t
$$

e dado que a derivada comuta com o operador convolução, temos que:

$$
\begin{aligned}
\int_{0}^{\infty}\left\|\theta_{t}\left(1-P_{t}^{2}\right) \nabla f\right\|_{L^{2}}^{2} \frac{d t}{t} & \leq C \int_{0}^{\infty}\left\|\theta_{t} \nabla\left(1-P_{t}^{2}\right) f\right\|_{L^{2}}^{2} \frac{d t}{t} \\
& \leq C \int_{0}^{\infty}\left\|\left(1-P_{t}^{2}\right) f\right\|_{L^{2}}^{2} \frac{d t}{t^{3}} \\
& =C \int_{0}^{\infty}\left\|\left(\widehat{1-P_{t}^{2}}\right) f\right\|_{L^{2}}^{2} \frac{d t}{t^{3}} \\
& =C \int_{0}^{\infty} \| \widehat{R_{t} \nabla f \|_{L^{2}}^{2}} \frac{d t}{t} \\
& =C \int_{0}^{\infty}\left\|R_{t} \nabla f\right\|_{L^{2}}^{2} \frac{d t}{t}
\end{aligned}
$$

Por (4.2) e pelo Lema B.5, a ultima desigualdade é estimada por:

$$
\int_{0}^{\infty}\left\|\theta_{t}\left(1-P_{t}^{2}\right) \nabla f\right\|_{L^{2}}^{2} \frac{d t}{t} \leq C\|\nabla f\|_{L^{2}}^{2} .
$$




\subsection{Redução à estimativa de medida Carleson}

Com o resultado anterior, temos que:

$$
\begin{aligned}
\int_{0}^{\infty}\left\|\theta_{t} \nabla f\right\|_{L^{2}}^{2} \frac{d t}{t} & \leq \int_{0}^{\infty}\left\|\theta_{t}\left(1-P_{t}^{2}\right) \nabla f\right\|_{L^{2}}^{2} \frac{d t}{t}+\int_{0}^{\infty}\left\|\theta_{t} P_{t}^{2} \nabla f\right\|_{L^{2}}^{2} \frac{d t}{t} \\
& \leq C\|\nabla f\|_{L^{2}}^{2}+\int_{0}^{\infty}\left\|\theta_{t} P_{t}^{2} \nabla f\right\|_{L^{2}}^{2} \frac{d t}{t} .
\end{aligned}
$$

Por tanto, para provar (4.1), precisamos somente de provar:

$$
\int_{0}^{\infty}\left\|\theta_{t} P_{t}^{2} \nabla f\right\|_{L^{2}}^{2} \frac{d t}{t} \leq C\|\nabla f\|_{L^{2}}^{2}
$$

Para reduzir nossas estimativas a uma estimativa de medida Carleson, começamos com uma definição para $\theta_{t}$ :

$$
\gamma_{t}(x)=\left(\theta_{t} \mathbf{1}\right)(x)=\left(\left(-\left(I+t^{2} L\right)^{-1} t \sum_{j} \frac{\partial}{\partial j} a_{j, k}\right)(x)\right)_{1}^{n}
$$

onde 1 é a matriz identidade, e a ação de $\theta_{t}$ sobre 1 é de coluna à coluna. Assim, segue que:

Lema 4.1 Dado $f \in L^{2}\left(\mathbb{R}^{n}\right)$ :

$$
\int_{0}^{\infty} \int_{\mathbb{R}^{n}}\left|\gamma_{t}(x)\left(P_{t}^{2} \nabla f\right)(x)-\theta_{t} P_{t}^{2} \nabla f(x)\right|^{2} \frac{d x d t}{t} \leq C\|\nabla f\|_{L^{2}}^{2}
$$

Mais ainda, se:

$$
\left\|\gamma_{t}\right\|^{2}:=\sup _{Q} \frac{1}{|Q|} \int_{Q}^{\ell(Q)} \int_{0}\left|\gamma_{t}(x)\right|^{2} \frac{d x d t}{t} \leq C\|\nabla f\|_{L^{2}}^{2}
$$

onde o supremo é tomado sobre todos os cubos $Q$ em $\mathbb{R}^{n}$, com lados paralelos aos eixos, então a desigualdade (4.4) mantêm-se com constante $C\left(1+\left\|\gamma_{t}^{2}\right\|\right)$. 
Prova: Suponha que (4.5) e (4.6) são satisfeitas; provaremos a última conclusão do lema 4.1: veja que o lado direito de (4.4) é limitado da seguinte maneira:

$$
\begin{aligned}
\int_{0}^{\infty}\left\|\theta_{t} P_{t}^{2} \nabla f\right\|_{L^{2}}^{2} \frac{d t}{t} & =\int_{0}^{\infty}\left\|\theta_{t} P_{t}^{2} \nabla f-\left(\gamma_{t} \cdot\left(P_{t}^{2} \nabla f\right)\right)+\left(\gamma_{t} \cdot\left(P_{t}^{2} \nabla f\right)\right)\right\|_{L^{2}}^{2} \frac{d t}{t} \\
& \leq \int_{0}^{\infty}\left\|\theta_{t} P_{t}^{2} \nabla f-\gamma_{t} \cdot\left(P_{t}^{2} \nabla f\right)\right\|_{L^{2}}^{2} \frac{d t}{t}+\int_{0}^{\infty}\left\|\gamma_{t} \cdot\left(P_{t}^{2} \nabla f\right)\right\|_{L^{2}}^{2} \frac{d t}{t} \\
& \leq C\|\nabla f\|_{L^{2}}^{2}+\int_{0}^{\infty} \int_{\mathbb{R}^{n}}\left|\gamma_{t}(x) \cdot\left(P_{t}^{2} \nabla f\right)(x)\right|^{2} \frac{d x d t}{t} \\
& \leq C\|\nabla f\|_{L^{2}}^{2}+C\left\|\gamma_{t}\right\|^{2}\|\nabla f\|_{L^{2}}^{2} \\
& =C\left(1+\left\|\gamma_{t}^{2}\right\|\right)\|\nabla f\|_{L^{2}}^{2} .
\end{aligned}
$$

onde usamos a desigualdade de Carleson, dada lema B.4, para estimar a penúltima fórmula. Com isso, a estimativa (K) foi provada. Ao longo desta seção, vamos nos focar em provar a desigualdade (4.5), assim que (4.6) será deixada a mostrar no seguinte capítulo.

Dado $t>0$, defina-se uma família de operadores:

$$
U_{t} \mathbf{f}:=\gamma_{t}(.) \cdot P_{t} \mathbf{f}-\theta_{t} P_{t} \mathbf{f}
$$

De modo que $U_{t} \mathbf{f}(x)=\gamma_{t}(x) \cdot P_{t} \mathbf{f}(x)-\theta_{t} P_{t} \mathbf{f}(x)$. Assim, se substituímos $\mathbf{f}$ por $P_{t} \nabla f$, com $f \in L^{2}\left(\mathbb{R}^{n}\right)$, obtém-se:

$$
\begin{aligned}
U_{t} P_{t} \nabla f(x) & =\gamma_{t}(x) \cdot P_{t}\left(P_{t} \nabla f\right)(x)-\theta_{t} P_{t}\left(P_{t} \nabla f\right)(x) \\
& =\gamma_{t}(x) \cdot P_{t}^{2} \nabla f(x)-\theta_{t} P_{t}^{2} \nabla f(x) .
\end{aligned}
$$

Por tanto, (4.5) é rescrito como:

$$
\int_{0}^{\infty}\left\|U_{t} P_{t} \nabla f\right\|_{L^{2}}^{2} \frac{d t}{t}=\int_{0}^{\infty} \int_{\mathbb{R}^{n}}\left|\gamma_{t}(x) \cdot P_{t}^{2} \nabla f(x)-\theta_{t} P_{t}^{2} \nabla f(x)\right|^{2} \frac{d t}{t} \leq C\|\nabla f\|_{L^{2}}^{2} .
$$

Note-se que (4.7) é a conclusão da proposição (2.3), aplicado para o operador:

$$
U_{t} P_{t}:\left(L^{2}\left(\mathbb{R}^{n}\right)\right)^{n} \longrightarrow L^{2}\left(\mathbb{R}^{n}\right)
$$

Logo, por essa proposição, é suficiente mostrar as hipóteses que envolvem ele, isto é, mostraremos que:

$$
\left\|U_{t} P_{t}\right\|_{\mathcal{B}\left(L^{2}\right)} \leq C, \quad \mathrm{e}
$$




$$
\left\|U_{t} P_{t} Q_{s}\right\|_{\mathcal{B}\left(L^{2}\right)} \leq K \min \left\{\frac{t}{s}, \frac{s}{t}\right\}^{\alpha}
$$

para algum $\alpha \in(0,1]$.

Observação: Veja que as normas acima na verdade estão definidas no espaço $\mathcal{B}\left(\left(L^{2}\left(\mathbb{R}^{n}\right)\right) n, L^{2}\left(\mathbb{R}^{n}\right)\right)$, mas por simplicidade escrevermos, daqui em diante, como:

$$
\|\quad\|_{\mathcal{B}\left(\left(L^{2}\left(\mathbb{R}^{n}\right)\right)^{n}, L^{2}\left(\mathbb{R}^{n}\right)\right)}=:\|\|_{\mathcal{B}\left(L^{2}\right)} .
$$

Provaremos primeiro a estimativa (4.8) a través dos seguintes dois lemas:

Lema 4.2 Para $t>0$, o operador $U_{t}$ é dado por um núcleo $U_{t}(x, y)$, tal que:

$$
U_{t} \mathbf{f}(x)=\int_{\mathbb{R}^{n}} U_{t}(x, y) \mathbf{f}(y) d y
$$

Mais ainda, $U_{t}$ é limitada em $L^{1}$ e existe $C=C(\lambda, \Lambda, n)>0$ tal que o núcleo $U_{t}(x, y)$ satisfaz:

$$
\sup _{y \in \mathbb{R}^{n}} \int_{\mathbb{R}^{n}}\left|U_{t}(x, y)\right| d x \leq C
$$

Demonstração: Considere um $t>0$ fixo, e sejam $\left\{Q_{k}^{t}\right\}_{k \in \mathbb{Z}^{n}}$ uma família que denota os cubos de lado $t$ com vértices em $t \mathbb{Z}^{n}$. Considere $\mathbf{f} \in\left[C_{0}^{\infty}\left(\mathbb{R}^{n}\right)\right]^{n}$ e seja $\mathbf{f}^{k}(x)=X_{Q_{k}^{t}} \mathbf{f}(x)$. Como $\rho(x) \in C_{0}^{\infty}\left(B_{1}(0)\right)$, segue que $\rho(x-y) \in B_{1}(x)$, logo $\rho\left(\frac{x-y}{t}\right) \in B_{t}(x)$. Com isso deduzimos que $\rho_{t}(x-y) \in B_{t}(x)$ e por tanto:

$$
\left|\rho\left(\frac{x-y}{t}\right)\right| \leq \frac{1}{t^{n}}\left|\rho\left(\frac{x-y}{t}\right)\right| \leq \frac{C}{t^{n}} \mathcal{X}_{B_{t}(x)}(y) .
$$

Veja também que $B_{t}(x) \subset 3 Q_{k}^{t}$, sem importar onde esteja $y \in Q_{k}^{t}$ e $|x-y| \leq t$. Segue então que:

$$
\begin{aligned}
\left|P_{t} \mathbf{f}^{k}(x)\right|=\left|\rho_{t} * \mathcal{X}_{Q_{k}^{t}} \mathbf{f}(x)\right| & =\left|\int_{\mathbb{R}^{n}} \rho_{t}(x-y) \cdot \mathcal{X}_{Q_{k}^{t}} \mathbf{f}(y) d y\right| \\
& \leq \int_{B_{t}(x)}\left|\rho_{t}(x-y)\right|\left|\mathcal{X}_{Q_{k}^{t}} \mathbf{f}(y)\right| d y \\
& \leq \frac{C}{t^{n}} \mathcal{X}_{B_{t}(y)}(x) \int_{B_{t}(x)}\left|\mathcal{X}_{Q_{k}^{t}} \mathbf{f}(y)\right| d y \\
& \leq \frac{C}{t^{n}} \mathcal{X}_{3 Q_{k}^{t}}(x) \int_{\mathbb{R}^{n}}\left|\mathcal{X}_{Q_{k}^{t}} \mathbf{f}(y)\right| d y \\
& =\frac{C}{t^{n}} \mathcal{X}_{3 Q_{k}^{t}}(x)\left\|\mathcal{X}_{Q_{k}^{t}} \mathbf{f}\right\|_{L^{1}}
\end{aligned}
$$


$\Rightarrow$

$$
\left|P_{t} \mathbf{f}^{k}(x)\right| \leq \frac{C}{t^{n}} \mathcal{X}_{3 Q_{k}^{t}}(x)\left\|\mathcal{X}_{Q_{k}^{t}} \mathbf{f}\right\|_{L^{1}}
$$

Pelo corolário 2.5 (o qual mantém-se tanto para cubos como para bolas), para todos os cubos $Q \subset \mathbb{R}^{n}$, tem-se a seguinte limitação uniforme:

$$
\frac{1}{|Q|} \int_{Q}\left|\gamma_{t}(x)\right|^{2} d x=\frac{1}{|Q|} \int_{Q}\left|\left(I+t^{2} L\right)^{-1} t \operatorname{div} A \mathbf{1}\right|^{2} d x \leq C\|\mathbf{1}\|=C
$$

Então:

$$
\begin{aligned}
\int_{3 Q_{k}^{t}}\left|\gamma_{t}(x)\right| d x & \leq\left(\int_{3 Q_{k}^{t}}\left|\gamma_{t}(x)\right|^{2} d x\right)^{\frac{1}{2}}\left(\int_{3 Q_{k}^{t}} d x\right)^{\frac{1}{2}} \\
& \leq C\left|Q_{k}^{t}\right|^{1 / 2} \cdot\left|3 Q_{k}^{t}\right|^{1 / 2} \\
& =C\left|Q_{k}^{t}\right| \\
& =C t^{n}
\end{aligned}
$$

Assim:

$$
\int_{3 Q_{k}^{t}}\left|\gamma_{t}(x)\right| d x \leq C t^{n}
$$

Agora, por (4.10) e (4.12), segue que

$$
\begin{aligned}
\int_{\mathbb{R}^{n}}\left|\gamma_{t}(x) \cdot P_{t} \mathbf{f}(x)\right| d x & =\int_{\mathbb{R}^{n}}\left|\sum_{k \in \|^{n}} \gamma_{t}(x) \cdot P_{t} \mathbf{f}_{k}(x)\right| d x \\
& \leq \int_{\mathbb{R}^{n}} \sum_{k \in \mathbb{Z}^{n}}\left|\gamma_{t}(x)\right|\left|P_{t} \mathbf{f}_{k}(x)\right| d x \\
& \leq \int_{\mathbb{R}^{n}} \sum_{k \in \mathbb{Z}^{n}}\left|\gamma_{t}(x)\right| \frac{C}{t^{n}} \mathcal{X}_{3 Q_{k}^{t}}(x)\left\|\mathcal{X}_{Q_{k}^{t}} \mathbf{f}\right\|_{L^{1}} d x \\
& \leq \sum_{k \in \mathbb{Z}^{n}} \frac{C}{t^{n}}\left\|\mathcal{X}_{Q_{k}^{t}} \mathbf{f}\right\|_{L^{1}} \int\left|\gamma_{t}(x)\right| \mathcal{X}_{3 Q_{k}^{t}}(x) d x \\
& \leq \sum_{k \in \mathbb{Z}^{n}} \frac{C}{t^{n}}\left\|\mathcal{X}_{Q_{k}^{t}} \mathbf{f}\right\|_{L^{1}} C t^{n} \\
& \leq \sum_{k \in \mathbb{Z}^{n}} C\left\|\mathcal{X}_{Q_{k}^{t}} \mathbf{f}\right\|_{L^{1}} \\
& \leq\|\mathbf{f}\|_{\mathbf{L}^{1}}
\end{aligned}
$$

Por tanto:

$$
\int_{\mathbb{R}^{n}}\left|\gamma_{t}(x) \cdot P_{t} \mathbf{f}(x)\right| d x \leq\|\mathbf{f}\|_{\mathbf{L}^{1}}
$$


para todos os cubos $Q_{k}^{t}$ que são disjuntos a menos de sua fronteira. Por outra parte, pela terceira desigualdade do lema 2.1 e por (4.10), temos que para todo $j, k, \operatorname{com} 3 Q_{k}^{t} \cap Q_{j}^{t}=\emptyset, j \neq k$ :

$$
\begin{aligned}
\int_{Q_{j}^{t}}\left|\theta_{t} P_{t} \mathbf{f}^{k}(x)\right|^{2} d x & =\int_{Q_{j}^{t}}\left|t\left(I+t^{2} L\right)^{-1} \operatorname{div} A \cdot P_{t} \mathbf{f}^{k}(x)\right|^{2} d x, \operatorname{com} \operatorname{supp} P_{t} \mathbf{f}^{k}(x) \subset 3 Q_{k}^{t} \\
& \leq C e^{-\frac{d\left(3 Q_{k}^{t}, Q_{j}^{t}\right)}{c t}} \int_{3 Q_{k}^{t}}\left|P_{t} \mathbf{f}^{k}(x)\right|^{2} d x \\
& \leq C e^{-\frac{|k-j|}{c}} \int_{3 Q_{k}^{t}} \frac{C}{t^{2 n}} \mathcal{X}_{3 Q_{k}^{t}}(x)^{2}\left\|\mathcal{X}_{Q_{k}^{t}} \mathbf{f}\right\|_{L^{1}}^{2} d x \\
& \leq C \frac{e^{-\frac{|k-j|}{c}}}{t^{2 n}}\left\|\mathcal{X}_{Q_{k}^{t}} \mathbf{f}\right\|_{L^{1}}^{2} \int_{3 Q_{k}^{t}} \mathcal{X}_{3 Q_{k}^{t}}(x)^{2} d x \\
& \leq C \frac{e^{-\frac{|k-j|}{c}}}{t^{2 n}}\left\|\mathcal{X}_{Q_{k}^{t}} \mathbf{f}\right\|_{L^{1}}^{2}\left|Q_{k}^{t}\right|
\end{aligned}
$$

Então:

$$
\begin{aligned}
\int_{Q_{j}^{t}}\left|\theta_{t} P_{t} \mathbf{f}^{k}(x)\right| d x & \leq\left(\int_{Q_{j}^{t}}\left|\theta_{t} P_{t} \mathbf{f}^{k}(x)\right|^{2} d x\right)^{1 / 2}\left(\int_{Q_{j}^{t}} 1 d x\right)^{1 / 2} \\
& \leq C \frac{e^{-\frac{|k-j|}{2 c}}}{t^{n}}\left\|\mathcal{X}_{Q_{k}^{t}} \mathbf{f}\right\|_{L^{1}}\left|Q_{k}^{t}\right|^{1 / 2} \cdot\left|Q_{k}^{t}\right|^{1 / 2} \\
& \leq C e^{-\frac{|k-j|}{2 c}}\left\|\mathcal{X}_{Q_{k}^{t}} \mathbf{f}\right\|_{L^{1}}
\end{aligned}
$$

Por tanto:

$$
\begin{aligned}
\int_{\mathbb{R}^{n}}\left|\theta_{t} P_{t} \mathbf{f}(x)\right| d x & \leq \sum_{k \in \mathbb{Z}^{n}} \sum_{j \in \mathbb{Z}^{n}} \int_{Q_{j}^{t}}\left|\theta_{t} P_{t} \mathbf{f}^{k}(x)\right| d x \\
& \leq C \sum_{k \in \mathbb{Z}^{n}} \sum_{j \in \mathbb{Z}^{n}} e^{-\frac{|k-j|}{2 c}}\left\|\mathcal{X}_{Q_{k}^{t}} \mathbf{f}\right\|_{L^{1}} \leq C\|\mathbf{f}\|_{\mathbf{L}^{1}} .
\end{aligned}
$$

Finalmente:

$$
\int_{\mathbb{R}^{n}}\left|U_{t} \mathbf{f}(x)\right| d x \leq \int_{\mathbb{R}^{n}}\left|\gamma_{t}(x) \cdot P_{t} \mathbf{f}(x)\right| d x+\int_{\mathbb{R}^{n}}\left|\theta_{t} P_{t} \mathbf{f}(x)\right| d x \leq C\|\mathbf{f}\|_{\mathbf{L}^{2}}, \quad \forall \mathbf{f} .
$$

Concluímos que $U_{t}$ é $L^{1}$-limitada. Agora, por o resultado do teorema 1.3.5 feito por Nelson Dunford em [17], segue que o operador $U_{t}$ é dado por um núcleo localmente integrável $K_{t}(x, y)=: U_{t}(x, y)$ satisfazendo:

$$
\sup _{y \in \mathbb{R}^{n}} \int_{\mathbb{R}^{n}}\left|U_{t}(x, y)\right| d x \leq C
$$


Lema 4.3 O núcleo do operador $U_{t} \mathbf{f}(x)=\gamma_{t}(x) \cdot \mathbf{f}(x)-\theta_{t} P_{t} \mathbf{f}(x)$, satisfaz:

$$
\int_{\mathbb{R}^{n}} e^{\frac{|x-y|}{4 c t}}\left|U_{t}(x, y)\right|^{2} d x \leq C \frac{\left|B_{t}(y)\right|}{t^{2 n}}
$$

onde Cé uma constante como no lema 2.1.

Demonstração: Seja $\phi \in C_{0}^{\infty}\left(B_{1}(0)\right), \phi \geq 0$ e $\int \phi=1$; Dado $\varepsilon>0$ e $y \in \mathbb{R}^{n}$, escrevemos:

$$
\varphi_{\varepsilon, y}(x):=\varphi_{\varepsilon}(x-y)=\varepsilon^{-n} \varphi\left(\frac{x-y}{\varepsilon}\right)
$$

Pelo lema 4.2 , sabemos que $U_{t}(x, y) \in L^{1}\left(\mathbb{R}^{n}\right)$ uniformemente; logo temos que:

$$
\left(U(\cdot, y) * \varphi_{\varepsilon}\right)(x) \longrightarrow U(x, y)
$$

quando $\varepsilon \rightarrow 0$, q.t.p. em $L^{1}, \forall y \in \mathbb{R}^{n}$.

Seja $R_{0}(y)=B_{2 t}(y)$, e $R_{j}(y)=B_{2^{j+1} t}(y) \backslash B_{2^{j} t}(y)$, para $j=1,2, \ldots$ (Anéis formados por diferencia de bolas). Então $R_{i}(y) \cap R_{j}(y)=\emptyset, i \neq j$ e

$$
\begin{aligned}
\int_{\mathbb{R}^{n}} e^{\frac{|x-y|}{4 c t}}\left|U_{t}\left(\varphi_{\varepsilon, y} \mathbf{I}\right)(x)\right|^{2} d x & \leq \sum_{j=0}^{\infty} \int_{R_{j}(y)} e^{\frac{|x-y|}{4 c t}}\left|U_{t}\left(\varphi_{\varepsilon, y} \mathbf{I}\right)(x)\right|^{2} d x \\
& \leq \sum_{j=0}^{\infty} e^{\frac{2^{j+1}}{4 c}} \int_{R_{j}(y)}\left|U_{t}\left(\varphi_{\varepsilon, y} \mathbf{I}\right)(x)\right|^{2} d x
\end{aligned}
$$

já que se $x \in R_{j}(y) \Rightarrow|x-y| \leq \sup |x-y| \leq 2^{j+1} t$. Note que, a priori, não sabemos se qualquer dos lados de $(4,15)$ são finitos. Para isso, considere primeiramente o caso $j=0$ :

$$
\begin{aligned}
\int_{R_{0}(y)}\left|U_{t}\left(\varphi_{\varepsilon, y} \mathbf{I}\right)(x)\right|^{2} d x & =\int_{R_{0}(y)}\left|\gamma_{t}(x) \cdot\left(\varphi_{\varepsilon, y} \mathbf{I}\right)(x)-\theta_{t} P_{t}\left(\varphi_{\varepsilon, y} \mathbf{I}\right)(x)\right|^{2} d x \\
& \leq 2 \int_{R_{0}(y)}\left|\gamma_{t}(x) \cdot\left(\varphi_{\varepsilon, y} \mathbf{I}\right)(x)\right|^{2} d x+2 \int_{R_{0}(y)}\left|\theta_{t} P_{t}\left(\varphi_{\varepsilon, y} \mathbf{I}\right)(x)\right|^{2} d x \\
& =2 \int_{B_{2 t}(y)}\left|\gamma_{t}(x) \cdot\left(\varphi_{\varepsilon, y} \mathbf{I}\right)(x)\right|^{2} d x+2 \int_{B_{2 t}(y)}\left|\theta_{t} P_{t}\left(\varphi_{\varepsilon, y} \mathbf{I}\right)(x)\right|^{2} d x
\end{aligned}
$$

Já que para $\varepsilon>0,\left|P_{t} \varphi_{\varepsilon, y}(x)\right| \leq C t^{-n} \mathcal{X}_{B_{2 t}(y)}(x)$, pelo corolário 2.5 e $(4,11)$ temos que:

$$
\int_{B_{2 t}(y)}\left|\gamma_{t}(x) \cdot P_{t}\left(\varphi_{\varepsilon, y} \mathbf{I}\right)(x)\right|^{2} d x \leq \int_{B_{2 t}(y)}\left|\gamma_{t}(x)\right|^{2}\left|P_{t}\left(\varphi_{\varepsilon, y} \mathbf{I}\right)(x)\right|^{2} d x \leq C t^{-2 n}\left|B_{t}(y)\right|
$$


Analogamente, usando o mesmo corolário:

$$
\int_{B_{2 t}(y)}\left|\theta_{t} P_{t}\left(\varphi_{\varepsilon, y} \mathbf{I}\right)(x)\right|^{2} d x \leq C\left\|P_{t}\left(\varphi_{\varepsilon, y} \mathbf{I}\right)\right\|_{\infty}^{2}\left|B_{t}(y)\right| \leq C t^{-2 n}\left|B_{t}(y)\right|
$$

Assim, para o caso $j=0, \int_{R_{0}(y)}\left|U_{t}\left(\varphi_{\varepsilon, y} \mathbf{I}\right)(x)\right|^{2} d x$ é limitada por $C t^{-2 n}\left|B_{t}(y)\right|$, Quando $j \geq 1$, segue que:

$$
\begin{aligned}
\int_{R_{j}(y)}\left|U_{t}\left(\varphi_{\varepsilon, y} \mathbf{I}\right)(x)\right|^{2} d x & =\int_{B_{2^{j+1} t}(y) \backslash B_{2^{j}}(y)}\left|\gamma_{t}(x) \cdot P_{t}\left(\varphi_{\varepsilon, y} \mathbf{I}\right)(x)-\theta_{t} P_{t}\left(\varphi_{\varepsilon, y} \mathbf{I}\right)(x)\right|^{2} d x \\
& =\int_{B_{2^{j+1} t}(y) \backslash B_{2^{j} t}(y)}\left|\theta_{t} P_{t}\left(\varphi_{\varepsilon, y} \mathbf{I}\right)(x)\right|^{2} d x \\
& =\int_{B_{2^{j+1}}(y) \backslash B_{2^{j}}(y)}\left|t\left(I+t^{2} L\right)^{-1} \operatorname{div} A P_{t}\left(\varphi_{\varepsilon, y} \mathbf{I}\right)(x)\right|^{2} d x
\end{aligned}
$$

Aqui o termo $P_{t}\left(\varphi_{\varepsilon, y} \mathbf{I}\right)(x)$ tem suporte em $B_{2 t}(y)$, e como a integral está sobre um conjunto que não contém ele, então $P_{t}\left(\varphi_{\varepsilon, y} \mathbf{I}\right)(x)=0$. Logo, pelas desigualdades do lema 2.1, temos, para $F=B_{2^{j+1} t}(y) \backslash B_{2^{j} t}(y)$ e $E=B_{2 t} y$

$$
\begin{aligned}
\int_{R_{j}(y)}\left|U_{t}\left(\varphi_{\varepsilon, y} \mathbf{I}\right)(x)\right|^{2} d x & \leq \int_{B_{2^{j}+1_{t}}(y) \backslash B_{2^{j} t}(y)}\left|t\left(I+t^{2} L\right)^{-1} \operatorname{div} A P_{t}\left(\varphi_{\varepsilon, y} \mathbf{I}\right)(x)\right|^{2} d x \\
& \leq \int_{B_{2 t} y} e^{-\frac{d i s t(E, F)}{c t}}\left|P_{t}\left(\varphi_{\varepsilon, y} \mathbf{I}\right)(x)\right|^{2} d x \\
& \leq \int_{B_{2 t} y} e^{-\frac{2^{j}}{c}}\left|P_{t}\left(\varphi_{\varepsilon, y} \mathbf{I}\right)(x)\right|^{2} d x \\
& \leq C t^{-2 n} e^{-\frac{2^{j}}{c}}\left|B_{2 t}(y)\right|
\end{aligned}
$$

Se ligarmos essas duas últimas estimativas no lado direito de (4.17), temos:

$$
\begin{array}{r}
\int_{\mathbb{R}^{n}} e^{\frac{|x-y|}{4 c t}}\left|U_{t}\left(\varphi_{\varepsilon, y} \mathbf{I}\right)(x)\right|^{2} d x \leq C t^{-2 n}\left|B_{t}(y)\right|\left(e^{\frac{2}{4 c}}+\sum_{j=1}^{\infty} e^{\frac{2^{j+1}}{4 c}} \cdot e^{-\frac{2^{j}}{c}}\right) \\
=C t^{-2 n}\left|B_{t}(y)\right|\left(e^{\frac{2}{4 c}}+\sum_{j=1}^{\infty} e^{-\frac{2^{j}}{2 c}}\right)
\end{array}
$$

onde a parte dentro do parenteses converge. Então, pelo lema de Fatou e (4.14) segue que:

$$
\int_{\mathbb{R}^{n}} e^{\frac{|x-y|}{4 c t}}\left|U_{t}(x, y)\right|^{2} d x \leq \lim \inf _{\varepsilon \rightarrow 0} \int_{\mathbb{R}^{n}} e^{\frac{|x-y|}{4 c t}}\left|U_{t}\left(\varphi_{\varepsilon, y} \mathbf{I}\right)(x)\right|^{2} d x \leq C t^{-2 n}\left|B_{t}(y)\right| .
$$


Agora provaremos (4.10). Como $P_{t}$ e $\theta_{t}$ são uniformemente $L^{2}$-limitados (pelo lema B.3 ou Lema 4.1 em [13] e o resultado ao inicio do capítulo, respectivamente), então para estabelecer a $L^{2}$-limitação de $U_{t}=\gamma_{t}(x) \cdot P_{t} \mathbf{f}-\theta_{t} P_{t} \mathbf{f}$ é suficiente estudar a $L^{2}$ - limitação de $\gamma_{t}(x) \cdot P_{t} \mathbf{f}$. Para isso, observe que:

$$
\begin{aligned}
\int_{\mathbb{R}^{n}}\left|\gamma_{t}(x) \cdot P_{t} \mathbf{f}(x)\right|^{2} d x & =\sum_{k \in \mathbb{Z}^{n}} \int_{Q_{k}^{t}}\left|\gamma_{t}(x) \cdot P_{t} \mathbf{f}^{k}(x)\right|^{2} d x \\
& \leq \sum_{k \in \mathbb{Z}^{n}} \int_{Q_{k}^{t}}\left|\gamma_{t}(x) \cdot P_{t}\left(\mathbf{f} \mathcal{X}_{3 Q_{k}^{t}}\right)(x)\right|^{2} d x
\end{aligned}
$$

onde a família $\left\{Q_{k}^{t}\right\}_{k \in \mathbb{Z}^{n}}$ da mesma forma como no lema 4.22. Para $x \in Q_{k}^{t}$, temos:

$$
\begin{aligned}
\left|P_{t}\left(\mathbf{f} \mathcal{X}_{3 Q_{k}^{t}}\right)(x)\right|^{2} & \leq\left|\int_{\mathbb{R}^{n}} \rho_{t}(x-y) \mathbf{f}(y) \mathcal{X}_{3 Q_{k}^{t}}(y) d y\right|^{2} \\
& \leq \int_{\mathbb{R}^{n}}\left|\rho_{t}(x-y) \mathbf{f}(y) \mathcal{X}_{3 Q_{k}^{t}}(y)\right|^{2} d y \\
& \leq \int_{3 Q_{k}^{t}}\left|\rho_{t}(x-y) \mathbf{f}(y)\right|^{2} d y \\
& \leq \frac{C}{t^{n}} \int_{3 Q_{k}^{t}}|\mathbf{f}(y)|^{2} d y .(?)
\end{aligned}
$$

Logo:

$$
\begin{aligned}
\int_{\mathbb{R}^{n}}\left|\gamma_{t}(x) \cdot P_{t} \mathbf{f}(x)\right|^{2} d x & \leq \sum_{k \in \mathbb{Z}^{n}} \int_{Q_{k}^{t}}\left|\gamma_{t}(x)\right|^{2}\left|P_{t}\left(\mathbf{f} \mathcal{X}_{3 Q_{k}^{t}}\right)(x)\right|^{2} d x \\
& \leq \sum_{k \in \mathbb{Z}^{n}} \sup _{x}\left|P_{t}\left(\mathbf{f} \mathcal{X}_{3 Q_{k}^{t}}\right)(x)\right|^{2} \int_{Q_{k}^{t}}\left|\gamma_{t}(x)\right|^{2} d x \\
& \leq \frac{C}{t^{n}} \cdot t^{n} \sum_{k \in \mathbb{Z}^{n}} \int_{3 Q_{k}^{t}}|\mathbf{f}(y)|^{2} d y \\
& \leq \sum_{k \in \mathbb{Z}^{n}} \int_{3 Q_{k}^{t}}|\mathbf{f}(y)|^{2} d y \\
& \leq C\|\mathbf{f}\|_{L^{2}} .
\end{aligned}
$$

Com isso, provamos que $U_{t}$ é limitada em $\left(L^{2}\left(\mathbb{R}^{n}\right)\right)^{n}$. Já que $P_{t}$ é uniformemente $L^{2}$-limitada por lema B.3, finalizamos a prova de (4.8). 
Para provar (4.9), e com isso finalizar a prova da redução à uma estimativa de medida Carleson dada pelo lema 4.1, vamos considerar como antes $Q_{s} f(x)=$ $\psi_{s} * f(x)$. Pelo análise de Fourier estândar (Veja-se [19]) temos $\left\|U_{t} P_{t} Q_{s}\right\|_{\mathcal{B}(\mathcal{L} \in)} \leq$ $C\left\|P_{t} Q_{s}\right\|_{\mathcal{B}(\mathcal{L} \in)} \leq C\left\{\frac{s}{t}\right\}^{\alpha}$, para alguma constante $\alpha$, com $s \leq t$. Assim, para provar (4.11), é suficiente estabelecer:

Lema 4.4 Temos que:

$$
\left\|U_{t} P_{t} Q_{s}\right\|_{\mathcal{B}\left(L^{2}\right)} \leq C\left(\frac{t}{s}\right)^{\alpha}
$$

para $t \leq s$ e algum $\alpha>0$.

Ideia da demonstração: Antes de dar a ideia da prova, vamos mostrar dois resultados que serão de ajuda:

Lema 4.5 $U_{t}(\mathbf{I})=0$ no sentido que $\left\langle U_{t}\left(\mathcal{X}_{R} \mathbf{I}\right), \mathbf{g}\right\rangle$ converge a 0 , quando $R \longrightarrow \infty$ para cada $\mathbf{g} \in\left(L^{2}\left(\mathbb{R}^{n}\right)\right)^{n}$, onde $\mathcal{X}_{R}$ é a função indicadora da bola $B_{R}=B_{R}(0)$.

Prova: Lembra-se que $U_{t}=\gamma_{t}(x) \cdot P_{t}-\theta_{t} P_{t}$. Logo, escrevemos

$$
\mathbf{g}=\mathcal{X}_{R / 4} \mathbf{g}+\left(1-\mathcal{X}_{R / 4}\right) \mathbf{g}
$$

Como para $R$ suficientemente grande, tem-se que $1-P_{t} \mathcal{X}_{R} \equiv 0$ sobre o suporte de $\mathcal{X}_{R / 4} \mathrm{~g}$ ( para isso, assuma que $R>4 t$, com $R$ suficientemente grande), temos:

$$
\begin{aligned}
\left\langle U_{t}\left(\mathcal{X}_{R} \mathbf{I}\right), \mathcal{X}_{R / 4} \mathbf{g}\right\rangle_{L^{2}}= & \left\langle\gamma_{t}(x) \cdot P_{t}\left(\mathcal{X}_{R} \mathbf{I}\right)-\theta_{t} P_{t}\left(\mathcal{X}_{R} \mathbf{I}\right)\right\rangle_{L^{2}} \\
= & -\int_{\mathbb{R}^{n}}\left(I+t^{2} L\right)^{-1} t \operatorname{div} A \mathbf{I}(x)\left(P_{t}\left(\mathcal{X}_{R} \mathbf{I}\right)\right)(x) \cdot \overline{\mathcal{X}_{R / 4} \mathbf{g}(x)} d x \\
& +\int_{\mathbb{R}^{n}}\left(I+t^{2} L\right)^{-1} t \operatorname{div}\left(A \cdot P_{t}\left(\mathcal{X}_{R} \mathbf{I}\right)\right)(x) \cdot \overline{\mathcal{X}_{R / 4} \mathbf{g}(x)} d x \\
= & -\int_{\mathbb{R}^{n}}\left(I+t^{2} L\right)^{-1} t \operatorname{div} A \mathbf{I}(x) \cdot \overline{\mathcal{X}_{R / 4} \mathbf{g}(x)} d x \\
& +\int_{\mathbb{R}^{n}}\left(I+t^{2} L\right)^{-1} t \operatorname{div}\left(A \cdot P_{t}\left(\mathcal{X}_{R} \mathbf{I}\right)\right)(x) \cdot \overline{\mathcal{X}_{R / 4} \mathbf{g}(x)} d x \\
= & -\int_{\mathbb{R}^{n}}\left(I+t^{2} L\right)^{-1} t \operatorname{div} A\left(\left(1-P_{t} \mathcal{X}_{R}\right) \mathbf{I}\right)(x) \cdot \overline{\mathcal{X}_{R / 4} \mathbf{g}(x)} d x \\
= & \int_{\mathbb{R}^{n}} A\left(\left(1-P_{t} \mathcal{X}_{R}\right) \mathbf{I}\right)(x) \cdot t \nabla\left(\left(I+t^{2} L\right)^{-1}\right)^{*} \overline{\mathcal{X}_{R / 4} \mathbf{g}(x)} d x
\end{aligned}
$$


Como assumimos que $R>4 t$, segue que:

$$
\begin{aligned}
\left|\left\langle U_{t}\left(\mathcal{X}_{R} \mathbf{I}\right), \mathcal{X}_{R / 4} \mathbf{g}\right\rangle\right| & =\left|\int_{\mathbb{R}^{n}} A\left(\left(1-P_{t} \mathcal{X}_{R}\right) \mathbf{I}\right)(x) \cdot t \nabla\left(\left(I+t^{2} L\right)^{-1}\right)^{*} \overline{\mathcal{X}_{R / 4} \mathbf{g}(x)} d x\right| \\
& \leq C \int_{\mathbb{R}^{n}}\left|\left(\left(1-P_{t} \mathcal{X}_{R}\right) \mathbf{I}\right)(x)\right|\left|t \nabla\left(\left(I+t^{2} L\right)^{-1}\right)^{*} \overline{\mathcal{X}_{R / 4} \mathbf{g}(x)}\right| d x \\
& \leq C \sum_{j=0}^{\infty} \int_{D_{j}}\left|\left(\left(1-P_{t} \mathcal{X}_{R}\right) \mathbf{I}\right)(x)\right|\left|t \nabla\left(\left(I+t^{2} L\right)^{-1}\right)^{*} \overline{\mathcal{X}_{R / 4} \mathbf{g}(x)}\right| d x
\end{aligned}
$$

onde $D_{j}=B_{2^{j} R} \backslash B_{2^{j-1} R}$. Observe também que fora de $B_{R / 4}, P_{t} \mathcal{X}_{R} \mathbf{I}(x)=0$.

Dessa forma

$$
\begin{aligned}
& \left|\left\langle U_{t}\left(\mathcal{X}_{R} \mathbf{I}\right), \mathcal{X}_{R / 4} \mathbf{g}\right\rangle\right| \leq C \sum_{j=0}^{\infty} \int_{D_{j}}\left|t \nabla\left(\left(I+t^{2} L\right)^{-1}\right)^{*} \overline{\mathcal{X}_{R / 4} \mathbf{g}(x)}\right| d x \\
& \leq C \sum_{j=0}^{\infty}\left(\int_{D_{j}}\left|t \nabla\left(\left(I+t^{2} L\right)^{-1}\right)^{*} \overline{\mathcal{X}_{R / 4} \mathbf{g}(x)}\right|^{2} d x\right)^{1 / 2}\left(\int_{D_{j}} 1 d x\right)^{1 / 2} \\
& \leq C \sum_{j=0}^{\infty}\left|B_{2^{j} R}\right|^{1 / 2}\left(\int_{D_{j}}\left|t \nabla\left(\left(I+t^{2} L\right)^{-1}\right)^{*} \overline{\mathcal{X}_{R / 4} \mathbf{g}(x)}\right|^{2} d x\right)^{1 / 2} \\
& \leq C \sum_{j=0}^{\infty}\left|B_{2^{j} R}\right|^{\frac{1}{2}}\left(e^{-\frac{d}{c t}} \int_{B_{R / 4}}\left|\mathcal{X}_{R / 4} \mathbf{g}(x)\right|^{2}\right)^{1 / 2} \\
& \leq C \sum_{j=0}^{\infty}\left|B_{2^{j} R}\right|^{\frac{1}{2}}\left(e^{-\frac{2^{j} R}{c t}} \int_{\mathbb{R}^{n}}|\mathbf{g}(x)|^{2}\right)^{1 / 2} \\
& =C\left|B_{R}\right|^{\frac{1}{2}} \sum_{j=0}^{\infty} 2^{\frac{j}{2}} e^{-\frac{m^{j} R}{2 c t}}\|\mathbf{g}\|_{L^{2}} \\
& =C\left|B_{R}\right|^{\frac{1}{2}}\|\mathbf{g}\|_{L^{2}} \sum_{j=0}^{\infty} e^{\ln \left(m^{\frac{j}{2}}\right)} e^{-\frac{2^{j} R}{2 c t}} \\
& =C\left|B_{R}\right|^{\frac{1}{2}}\|\mathbf{g}\|_{L^{2}} \sum_{j=0}^{\infty} \exp \left[-\left(\frac{2^{j} R}{2 c t}-\frac{\ln m}{2} j\right)\right]
\end{aligned}
$$

onde $m$ é obtida de $\left|B_{2^{j} R}\right| \leq m^{j}\left|B_{R}\right|$. O lado direito da estimativa do exponencial, tende à zero quando $R$ tende à $\infty$. 
Por outro lado, por lema 4.3, temos:

$$
\begin{aligned}
& \left|\left\langle U_{t}\left(\mathcal{X}_{R} \mathbf{I}\right),\left(1-\mathcal{X}_{R / 4}\right) \mathbf{g}\right\rangle\right|=\left|\int_{\mathbb{R}^{n}}\left(U_{t} \mathcal{X}_{R} \mathbf{I}\right)(x) \cdot \overline{\left(1-\mathcal{X}_{R / 4}\right) \mathbf{g}(x)} d x\right| \\
& =\int_{\mathbb{R}^{n} \backslash B_{R / 4}}\left|\left(\int_{B_{R}} U_{t}(x, y) \mathbf{I} d y\right) \cdot \overline{\mathbf{g}(x)}\right| d x \\
& \leq\left(\int_{\mathbb{R}^{n} \backslash B_{R / 4}}|\mathbf{g}(x)|^{2} d x\right)^{1 / 2}\left(\int_{\mathbb{R}^{n} \backslash B_{R / 4}}\left|\int_{B_{R}} U_{t}(x, y) \mathbf{I} d y\right|^{2} d x\right)^{1 / 2} \\
& \leq\|\mathbf{g}\|_{L^{2}}\left(\int_{\mathbb{R}^{n} \backslash B_{R / 4}}\left|\int_{B_{R}} U_{t}(x, y) e^{\frac{|x-y|}{16 c t}} e^{-\frac{|x-y|}{16 c t}} d y\right|^{2} d x\right)^{1 / 2} \\
& \leq\|\mathbf{g}\|_{L^{2}}\left(\int_{\mathbb{R}^{n} \backslash B_{R / 4}}\left(\int_{B_{R}}\left|U_{t}(x, y)\right|^{2} e^{\frac{|x-y|}{8 c t}} d y\right)\left(\int_{B_{R}} e^{-\frac{|x-y|}{8 c t}} d y\right) d x\right)^{1 / 2} \\
& \leq\|\mathbf{g}\|_{L^{2}}\left(e^{-\frac{R}{32 c t}}\left|B_{R}\right| \int_{\mathbb{R}^{n} \backslash B_{R / 4}}\left(\int_{B_{R}}\left|U_{t}(x, y)\right|^{2} e^{\frac{|x-y|}{8 c t}} d y\right) d x\right)^{1 / 2} \\
& \leq\|\mathbf{g}\|_{L^{2}}\left(e^{-\frac{R}{32 c t}}\left|B_{R}\right| \int_{B_{R}}\left(\int_{\mathbb{R}^{n}}\left|U_{t}(x, y)\right|^{2} e^{\frac{|x-y|}{4 c t}} d x\right) d y\right)^{1 / 2} \\
& \leq\|\mathbf{g}\|_{L^{2}}\left(C e^{-\frac{R}{32 c t}}\left|B_{R}\right| \int_{B_{R}} \frac{\left|B_{t}(y)\right|}{t^{n}} d y\right)^{1 / 2} \\
& \leq\|\mathbf{g}\|_{L^{2}} C t^{-n / 2} e^{-\frac{R}{64 c t}}\left|B_{R}\right|^{1 / 2}\left(\int_{B_{R}}\left|B_{t}(y)\right| d y\right)^{1 / 2} \\
& \leq\|\mathbf{g}\|_{L^{2}} C t^{-n / 2} e^{-\frac{R}{64 c t}}\left|B_{R}\right|^{1 / 2}\left(\int_{B_{t}} \int_{B_{R}}(x+y) d y d x\right)^{1 / 2} \\
& \leq\|\mathbf{g}\|_{L^{2}} C e^{-\frac{R}{64 c t}}\left|B_{2 R}\right| .
\end{aligned}
$$

O qual tende à zero quando $R$ tende à $\infty$. Logo, Lema 4.5 segue do fato que:

$$
\left|\left\langle U_{t}\left(\mathcal{X}_{R} \mathbf{I}\right), \mathbf{g}\right\rangle\right| \leq\left|\left\langle U_{t}\left(\mathcal{X}_{R} \mathbf{I}\right), \mathcal{X}_{R / 4} \mathbf{g}\right\rangle\right|+\left|\left\langle U_{t}\left(\mathcal{X}_{R} \mathbf{I}\right),\left(1-\mathcal{X}_{R / 4}\right) \mathbf{g}\right\rangle\right|
$$

Assim, esse lema é provado. 
Corolário 4.6 Defina-se por $K_{t} \mathbf{f}(x)=U_{t}^{*} U_{t} \mathbf{f}(x)$, onde $U_{t}^{*}$ denota o operador adjunto de $U_{t}$ em $L^{2}\left(\mathbb{R}^{n}\right)$; Então $K_{t}$ é dado por um núcleo $K_{t}(x, y)$ com respeito à medida de Lebesgue, satisfazendo a seguinte limitação superior pontual:

$$
\left|K_{t}(x, y)\right| \leq \frac{C e^{-\frac{|x-y|}{9 c t}}}{t^{2 n}}\left|B_{t}(x)\right|^{1 / 2}\left|B_{t}(y)\right|^{1 / 2} .
$$

Demonstração: Primeiramente, observa-se que:

$$
\begin{aligned}
K_{t} \mathbf{f}(x)=U_{t}^{*} U_{t} \mathbf{f}(x) & =\int_{\mathbb{R}^{n}} U_{t}^{*}(x, z)\left(U_{t} \mathbf{f}\right)(z) d z \\
& =\int_{\mathbb{R}^{n}} \overline{U_{t}(z, x)} \int_{\mathbb{R}^{n}} U_{t}(z, y) \mathbf{f}(y) d y d z \\
& =\int_{\mathbb{R}^{n}} \int_{\mathbb{R}^{n}} \overline{U_{t}(z, x)} U_{t}(z, y) \mathbf{f}(y) d y d z \\
& =\int_{\mathbb{R}^{n}} \int_{\mathbb{R}^{n}} \overline{U_{t}(z, x)} U_{t}(z, y) d z \mathbf{f}(y) d y
\end{aligned}
$$

Logo, temos que $K_{t}(x, y)=\int_{\mathbb{R}^{n}} \overline{U_{t}(z, x)} U_{t}(z, y) d z$. Como $U_{t}$ pode ser escrito como $U_{t}=\left(U_{t}^{1}, U_{t}^{2}, \ldots, U_{t}^{n}\right), \operatorname{com} \mathbf{f}=\left(f_{1}, \ldots, f_{n}\right)$, segue então que:

$$
\left|K_{t}(x, y)\right| \leq \sum_{j, l=1}^{n} \int_{\mathbb{R}^{n}}\left|\overline{U_{t}^{j}(z, x)}\right|\left|U_{t}^{l}(z, y)\right| d z
$$

Daí, por Lema 4.3, obtemos:

$$
\begin{aligned}
e^{\frac{|x-y|}{9 c t}}\left|K_{t}(x, y)\right| & \leq \sum_{j, l=1}^{n} \int_{\mathbb{R}^{n}} e^{\frac{|x-y|}{9 c t}}\left|\overline{U_{t}^{j}(z, x)}\right|\left|U_{t}^{l}(z, y)\right| d z \\
& \leq \sum_{j, l=1}^{n} \int_{\mathbb{R}^{n}} e^{\frac{|x-z+z-y|}{8 c t}}\left|\overline{U_{t}^{j}(z, x)}\right|\left|U_{t}^{l}(z, y)\right| d z \\
& \leq \sum_{j, l=1}^{n} \int_{\mathbb{R}^{n}} e^{\frac{|x-z|}{8 c t}}\left|\overline{U_{t}^{j}(z, x)}\right| e^{\frac{|z-y|}{8 c t}}\left|U_{t}^{l}(z, y)\right| d z \\
& \leq \sum_{j, l=1}^{n}\left(\int_{\mathbb{R}^{n}} e^{\frac{|x-z|}{4 c t}}\left|\overline{U_{t}^{j}(z, x)}\right|^{2} d z\right)^{1 / 2}\left(\int_{\mathbb{R}^{n}} e^{\frac{|y-z|}{4 c t}}\left|U_{t}^{l}(z, y)\right|^{2} d z\right)^{1 / 2} \\
& \leq \sum_{j, l=1}^{n} \frac{1}{t^{n}}\left|B_{t}(x)\right|^{1 / 2}\left|B_{t}(y)\right|^{1 / 2} \\
& \leq \frac{C}{t^{2 n}}\left|B_{t}(x)\right|^{1 / 2}\left|B_{t}(y)\right|^{1 / 2}
\end{aligned}
$$

Dessa forma:

$$
\left|K_{t}(x, y)\right| \leq \frac{C e^{-\frac{|x-y|}{9 c t}}}{t^{2 n}}\left|B_{t}(x)\right|^{1 / 2}\left|B_{t}(y)\right|^{1 / 2} \cdot \square
$$


Ideia da demonstração do Lema 4.4. Primeiramente, note que $K_{t}=U_{t}^{*} U_{t}$ satisfaz a propriedade de conservação no sentido do Lema 4.5. Isto segue do Lema 4.5, por dualidade e a limitação $L^{2}$-uniforme de $\left\|U_{t} P_{t}\right\|$. Então, podemos escrever:

$$
\begin{aligned}
K_{t} Q_{s} \mathbf{f}(x) & =\int_{\mathbb{R}^{n}} \int_{\mathbb{R}^{n}} K_{t}(x, y) \psi_{s}(y-z) \mathbf{f}(z) d z d y \\
& =\int_{\mathbb{R}^{n}} \int_{\mathbb{R}^{n}} K_{t}(x, y)\left(\psi_{s}(y-z)-\psi_{s}(x-z)\right) d z \mathbf{f}(z) d y
\end{aligned}
$$

Já que

$$
\left|\psi_{s}(y-z)-\psi_{s}(x-z)\right| \leq \frac{t}{s} s^{-n} \frac{|y-x|}{t}\left(\mathcal{X}_{B_{s}(x)}(z)+\mathcal{X}_{B_{s}(y)}(z)\right)
$$

Usando o corolário 4.6 temos:

$$
\left|K_{t} Q_{s} \mathbf{f}(x)\right| \leq \frac{t}{s} \frac{\left|B_{t}(x)\right|}{t^{2 n}} \Theta_{s} \mathbf{f}(x) \int_{\mathbb{R}^{n}} e^{-\frac{|x-y|}{10 c t}} d y+\frac{t}{s} \frac{\left|B_{t}(x)\right|}{t^{2 n}} \int_{\mathbb{R}^{n}} e^{-\frac{|x-y|}{10 c t}} \Theta_{s} \mathbf{f}(y) d y
$$

Onde $\Theta_{s} f(x)=\frac{1}{\left|B_{s}(x)\right|} \int_{B_{s}(x)}|f(y)| d y$. Usando a teoria de Littlewood-Paley e o operador maximal de Hardy-Littlewood com $0<t \leq s$, temos as $L^{2}$-estimativas dos termos acima, limitadas somente por $\frac{t}{s}\|\mathbf{f}\|_{L^{2}}$ (Veja-se [19], para uma melhor compreensão). Assim:

$$
\left\|K_{t} Q_{s} \mathbf{f}\right\|_{L^{2}}=\left\|U_{t}^{*} U_{t} Q_{s} \mathbf{f}\right\|_{L^{2}} \leq \frac{t}{s}\|\mathbf{f}\|_{L^{2}}
$$

Logo, veja que:

$$
\begin{aligned}
\left\|U_{t} Q_{s} \mathbf{f}\right\|_{L^{2}}^{2} & =\left\langle U_{t} Q_{s} \mathbf{f}, U_{t} Q_{s} \mathbf{f}\right\rangle \\
& =\left\langle U_{t}^{*} U_{t} Q_{s} \mathbf{f}, Q_{s} \mathbf{f}\right\rangle \\
& \leq\left\|U_{t}^{*} U_{t} Q_{s} \mathbf{f}\right\|_{L^{2}}\left\|Q_{s} \mathbf{f}\right\|_{L^{2}} \\
& \leq \frac{t}{s}\|\mathbf{f}\|_{L^{2}}\left\|Q_{s} \mathbf{f}\right\|_{L^{2}} \\
& \leq \frac{t}{s}\|\mathbf{f}\|_{L^{2}}
\end{aligned}
$$

$\Rightarrow\left\|U_{t} Q_{s}\right\|_{\mathcal{B}\left(L^{2}\right)}^{2} \leq \frac{t}{s}$. Finalmente, como $P_{t} Q_{s} \mathbf{f}=Q_{s} P_{t} \mathbf{f}$ é limitado em $L^{2}\left(\mathbb{R}^{n}\right)$, temos:

$$
\left\|U_{t} P_{t} Q_{s} \mathbf{f}\right\|_{L^{2}}^{2}=\left\|U_{t} Q_{s} P_{t} \mathbf{f}\right\|_{L^{2}}^{2} \leq \frac{t}{s}\left\|P_{t} \mathbf{f}\right\|_{L^{2}}^{2} \leq C \frac{t}{s}\|\mathbf{f}\|_{L^{2}}^{2} .
$$

Com o qual Lema 4.4 está provado; e ,junto com a outra estimativa já obtida para $s \leq t$, obtemos (4.9). 


\section{Capítulo 5}

\section{$\mathrm{O}$ argumento $\mathrm{T}(\mathrm{b})$}

\subsection{O Teorema $\mathrm{T}(\mathrm{b})$ e resultados preliminares}

O teorema $\mathrm{T}(\mathrm{b})$ é um critério de limitação para operadores integrais singulares que atuam sobre funções limitadas invertíveis e acretivas, isto é, operadores da forma:

$$
T f(x)=\int K(x, y) f(y) d y
$$

onde as propriedades de $K(x, y)$ foram vistas no capítulo anterior.

Mais precisamente, procuramos, por meio de critérios e resultados que envolvem resultados do capítulo 2, a $L^{2}$-limitação de esses operadores que, como visto anteriormente, satisfazem condições de "bom comportamento"sobre um certo conjunto de funções teste apropriadas não degeneradas $b$. Em essência, a limitação é testada sobre um subconjunto de funções em $L^{2}$, e a limitação é inferida para todas as funções de $L^{2}\left(\mathbb{R}^{n}\right)$. Tais funções teste são funções de colisão adequadas adaptadas a intervalos (no caso unidimensional) ou "cubos" (várias dimensões) e este principio de localização decorre das propriedades de localização dos núcleos integrais singulares sobre $\mathbb{R}^{n}$.

Neste capitulo, finalizarmos a demonstração da conjetura proposta por T.Kato, resolvendo $(4,6)$ e, por conseguinte, a estimativa $(\mathrm{K})$. Para isso, precisarmos de alguns preliminares que fornecem umas estimativas que facilitam o desenvolvimento deste capitulo.

Seja um cubo $Q$ (da mesma maneira que foi tomado para o lema 2.3) em $\mathbb{R}^{n}$, e considere uma coleção de cubos diádicos em $\mathbb{R}^{n}$ que contém $Q$, ou seja:

$$
\mathcal{D}:=\left\{Q=2^{n}\left([0.1)^{n}+k\right), k \in \mathbb{Z}^{n}\right\} .
$$


Para cada cubo $Q^{\prime}=Q_{\mathcal{D}}^{\prime}(x) \in \mathcal{D}$ tal que $x \in Q^{\prime}$ e $\frac{1}{2} \ell\left(Q^{\prime}\right)<t \leq \ell\left(Q^{\prime}\right)$, defina-se o seu correspondente operador de média diádica

$$
S_{t}^{Q} \mathbf{f}(x)=\frac{1}{\left|Q^{\prime}\right|} \int_{Q^{\prime}} \mathbf{f}(y) d y=\frac{1}{\left|Q^{\prime}\right|} \int_{Q_{\mathcal{D}}^{\prime}(x)} \mathbf{f}(y) d y
$$

Assim:

Lema 5.1 Para alguma constante $C$ dependendo somente de $n, \lambda, e \Lambda$, temos:

$$
\int_{Q} \int_{0}^{\ell(Q)}\left|\gamma_{t}(x) \cdot\left(\left(S_{t}^{Q}-P_{t}^{2}\right) \mathbf{f}\right)(x)\right|^{2} \frac{d x d t}{t} \leq C \int_{\mathbb{R}^{n}}|\mathbf{f}(x)|^{2} d x
$$

Demonstração: Veja [7] ou [12]. Ambos fazem a prova em base a Lema B.4 e B.5.

\section{$5.2 \quad \mathrm{O}$ argumento $\mathrm{T}(\mathrm{b})$}

Esta ultima seção vai ser dedicada a mostrar a estimativa de medida Carleson pendente (4.6), para finalmente concluir a demonstração de (K). Lembrando que a estimativa restante é dada por:

$$
\sup _{Q} \frac{1}{|Q|} \int_{Q}^{\ell(Q)} \int_{0}^{\ell}\left|\gamma_{t}(x)\right|^{2} \frac{d x d t}{t}<\infty
$$

onde $\gamma_{t}(x)=\left(-\left(I+t^{2} L\right)^{-1} t \operatorname{div} A \mathbf{1}(x)\right)_{1}^{n}$. Começaremos enunciando algumas definições:

Definição 5.2 Dado um cubo arbitrário $Q$, com centro $x_{Q}$, defina-se:

$$
\Phi_{Q}(x)=x-x_{Q}
$$

Então $\gamma_{t}(x)=\theta_{t} \nabla \Phi_{Q}(x)=-\left(I+t^{2} L\right)^{-1} t \operatorname{div} A \nabla \Phi_{Q}=-\left(I+t^{2} L\right)^{-1} t L \Phi_{Q}$

Fixando um cubo $Q \in \mathcal{D}$, dado $\varepsilon \in(0,1)$, e dado um vetor unitário $w \in \mathbb{C}^{n}$, definimos uma função escalar:

$$
f_{Q, w}^{\varepsilon}:=\left(1+(\varepsilon \ell(Q))^{2} L\right)^{-1}\left(\Phi_{Q} \cdot w^{*}\right)
$$

Lembrando de algumas estimativas obtidas no capítulo 2, vemos que do lema 2.3, deduzimos facilmente que:

$$
\int_{5 Q}\left|f_{Q, w}^{\varepsilon}-\Phi_{Q} \cdot w^{*}\right|^{2} d x \leq C_{1} \varepsilon^{2} \ell(Q)^{2}|Q|
$$


e

$$
\int_{5 Q}\left|\nabla\left(f_{Q, w}^{\varepsilon}-\Phi_{Q} \cdot w^{*}\right)\right|^{2} d x \leq C_{2}|Q|
$$

Basta substituir $t$ por $\ell(Q)$ para obter os resultados desejados. Aqui, $C_{1}$ e $C_{2}$ dependem somente de $n, \lambda$ e $\Lambda$ e NÃO de $\varepsilon, Q$ e $w$. É de importante observação que as constantes $C_{1}$ e $C_{2}$ acima são independentes de $\varepsilon$. A prova de (4.6) segue imediatamente a partir da combinação dos seguintes dois lemas e o resto desta seção será dedicado a prová-los.

Lema 5.3 Existe um $\varepsilon>0$ dependendo somente de $n, \lambda$ e $\Lambda$ e um conjunto finito $W$ de vetores unitários em $\mathbb{C}^{n}$ cuja cardinalidade depende apenas de $\varepsilon$ e $n$, tal que:

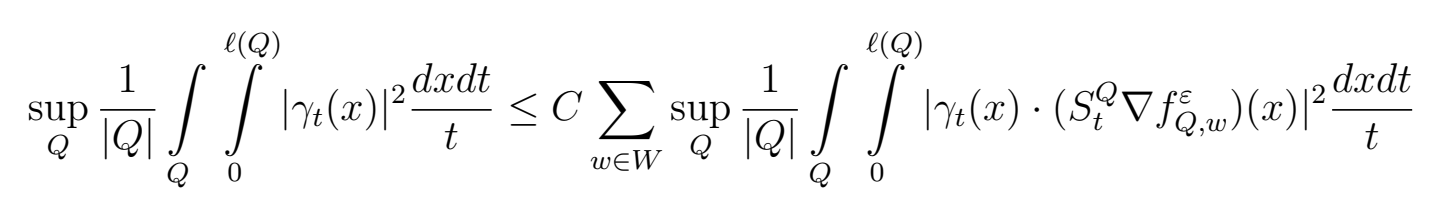

onde $C$ depende apenas de $n, \lambda$ e $\Lambda$.

Lema 5.4 Para $C$ dependendo apenas de $n, \lambda$ e $\Lambda$ e $\varepsilon>0$, temos:

$$
\int_{Q}^{\ell} \int_{0}^{\ell(Q)}\left|\gamma_{t}(x) \cdot\left(S_{t}^{Q} \nabla f_{Q, w}^{\varepsilon}\right)(x)\right|^{2} \frac{d x d t}{t} \leq C|Q|
$$

Prova do lema 5.4: Tome-se uma função suavizante de corte $\mathcal{X}=\mathcal{X}_{Q}$ localizada em $4 Q$ e igual à 1 em $2 Q$ com a propriedade $\|\mathcal{X}\|_{\infty}+\ell(Q)\|\nabla \mathcal{X}\|_{\infty} \leq c=c(n)$. Para $x \in Q$, temos que $S_{t}^{Q} \nabla f=S_{t}^{Q} \nabla \mathcal{X} f(x)$, pois:

$$
S_{t}^{Q} \mathcal{X} \nabla f=\frac{1}{\left|Q^{\prime}\right|} \int_{Q^{\prime}} \nabla(\mathcal{X} f)(y) d y=\frac{1}{\left|Q^{\prime}\right|} \int_{Q^{\prime}} \mathcal{X} \nabla f(x) d y=\frac{1}{\left|Q^{\prime}\right|} \int_{Q^{\prime}} \nabla f(y) d y=S_{t}^{Q} \nabla f
$$


Logo, tem-se que:

$$
\begin{aligned}
\int_{Q} \int_{0}^{\ell(Q)}\left|\gamma_{t}(x) \cdot\left(S_{t}^{Q} \nabla f_{Q, w}^{\varepsilon}\right)(x)\right|^{2} \frac{d x d t}{t} & =\int_{Q} \int_{0}^{\ell(Q)}\left|\gamma_{t}(x) \cdot\left(S_{t}^{Q} \nabla \mathcal{X} f_{Q, w}^{\varepsilon}\right)(x)\right|^{2} \frac{d x d t}{t} \\
& =\int_{Q}^{\ell} \int_{0}^{\ell(Q)}\left|\gamma_{t}(x) \cdot\left(\left(S_{t}^{Q}-P_{t}^{2}+P_{t}^{2}\right) \nabla \mathcal{X} f_{Q, w}^{\varepsilon}\right)(x)\right|^{2} \frac{d x d t}{t} \\
& \leq 2 \int_{Q}^{\ell(Q)} \int_{0}^{\ell(Q)}\left|\gamma_{t}(x) \cdot\left(\left(S_{t}^{Q}-P_{t}^{2}\right) \nabla \mathcal{X} f_{Q, w}^{\varepsilon}\right)(x)\right|^{2} \frac{d x d t}{t}+ \\
& 2 \int_{Q}^{\ell} \int_{0}^{\left(\gamma_{t}\right.}\left|\gamma_{t}(x) \cdot\left(P_{t}^{2} \nabla\left(\mathcal{X} f_{Q, w}^{\varepsilon}\right)\right)(x)\right|^{2} \frac{d x d t}{t} .
\end{aligned}
$$

Observe que, pelo Lema 5.1, o primeiro somando do lado direito da ultima desigualdade, é limitado por

$$
C \int_{\mathbb{R}^{n}}\left|\nabla\left(\mathcal{X} f_{Q, w}^{\varepsilon}\right)(x)\right|^{2} d x .
$$

é o integrando do segundo fator pode ser decomposto da seguinte maneira:

$$
\begin{aligned}
\left|\gamma_{t}(x) \cdot\left(P_{t}^{2} \nabla\left(\mathcal{X} f_{Q, w}^{\varepsilon}\right)\right)\right|^{2}= & \left|\gamma_{t}(x) \cdot\left(P_{t}^{2} \nabla\left(\mathcal{X} f_{Q, w}^{\varepsilon}\right)\right) \pm \theta_{t} P_{t}^{2} \nabla\left(\mathcal{X} f_{Q, w}^{\varepsilon}\right) \pm \theta_{t} \nabla\left(\mathcal{X} f_{Q, w}^{\varepsilon}\right)\right|^{2} \\
\leq & 4\left|\gamma_{t}(x) \cdot\left(P_{t}^{2} \nabla\left(\mathcal{X} f_{Q, w}^{\varepsilon}\right)\right)-\theta_{t} P_{t}^{2} \nabla\left(\mathcal{X} f_{Q, w}^{\varepsilon}\right)\right|^{2}+ \\
& 4\left|\theta_{t}\left(1-P_{t}^{2}\right) \nabla\left(\mathcal{X} f_{Q, w}^{\varepsilon}\right)\right|^{2}+ \\
& 2\left|\theta_{t} \nabla\left(\mathcal{X} f_{Q, w}^{\varepsilon}\right)\right|^{2}
\end{aligned}
$$

Veja que voltando à integral inicial, obtém-se:

$$
\begin{aligned}
2 \int_{Q} \int_{0}^{\ell(Q)}\left|\gamma_{t}(x) \cdot\left(P_{t}^{2} \nabla \mathcal{X} f_{Q, w}^{\varepsilon}\right)\right|^{2} \frac{d x d t}{t} \leq & 8 \int_{Q} \int_{0}^{\ell(Q)}\left|\gamma_{t}(x) \cdot\left(P_{t}^{2} \nabla \mathcal{X} f_{Q, w}^{\varepsilon}\right)-\theta_{t} P_{t}^{2}\left(\nabla \mathcal{X} f_{Q, w}^{\varepsilon}\right)\right|^{2} \frac{d x d t}{t}+ \\
& 8 \int_{Q}^{\ell} \int_{0}^{\ell(Q)}\left|\theta_{t}\left(1-P_{t}^{2}\right) \nabla\left(\mathcal{X} f_{Q, w}^{\varepsilon}\right)\right|^{2} \frac{d x d t}{t}+ \\
& 4 \int_{Q}^{\ell(Q)} \int_{0}^{\ell)}\left|\theta_{t} \nabla\left(\mathcal{X} f_{Q, w}^{\varepsilon}\right)\right|^{2} \frac{d x d t}{t} .
\end{aligned}
$$

O primeiro e segundo que têm constante 8, é limitado, pelas estimativas (4.5) e (4.3) respetivamente, por:

$$
C \int_{\mathbb{R}^{n}}\left|\nabla\left(\mathcal{X} f_{Q, w}^{\varepsilon}\right)(x)\right|^{2} d x .
$$


Reduzindo, temos:

$$
\begin{array}{r}
\int_{Q} \int_{0}^{\ell(Q)}\left|\gamma_{t}(x) \cdot\left(S_{t}^{Q} \nabla f_{Q, w}^{\varepsilon}\right)(x)\right|^{2} \frac{d x d t}{t} \leq C \int_{\mathbb{R}^{n}}\left|\nabla\left(\mathcal{X} f_{Q, w}^{\varepsilon}\right)(x)\right|^{2} d x+ \\
4 \int_{Q}^{\ell(Q)} \int_{0}^{\ell}\left|\theta_{t} \nabla\left(\mathcal{X} f_{Q, w}^{\varepsilon}\right)(x)\right|^{2} \frac{d x d t}{t}
\end{array}
$$

Nossa tarefa é, por tanto, limitar a ultima expressão por $C|Q|$. Primeiramente, vamos estimar $\int_{\mathbb{R}^{n}}\left|\nabla\left(\mathcal{X} f_{Q, w}^{\varepsilon}\right)\right|^{2} d x$. De fato:

$$
\begin{aligned}
& \int_{\mathbb{R}^{n}}|\nabla(\mathcal{X})(x)|^{2} d x \leq 2 \int_{\mathbb{R}^{n}}\left|f_{Q, w}^{\varepsilon} \nabla \mathcal{X}(x)\right|^{2} d x+2 \int_{\mathbb{R}^{n}}\left|\mathcal{X} \nabla\left(f_{Q, w}^{\varepsilon}\right)(x)\right|^{2} d x \\
& \leq 2 \int_{4 Q}\left|f_{Q, w}^{\varepsilon} \nabla \mathcal{X}(x)\right|^{2} d x+2 \int_{4 Q}\left|\mathcal{X} \nabla\left(f_{Q, w}^{\varepsilon}\right)(x)\right|^{2} d x \\
& \leq 2\|\nabla \mathcal{X}\|_{\infty}^{2} \int_{4 Q}\left|f_{Q, w}^{\varepsilon}(x)\right|^{2} d x+2\|\mathcal{X}\|_{\infty}^{2} \int_{4 Q}\left|\nabla\left(f_{Q, w}^{\varepsilon}\right)(x)\right|^{2} d x \\
& \leq \frac{c(n)}{\ell(Q)^{2}} \int_{4 Q}\left|f_{Q, w}^{\varepsilon}(x)\right|^{2} d x+c(n) \int_{4 Q}\left|\nabla\left(f_{Q, w}^{\varepsilon}\right)(x)\right|^{2} d x \\
& \leq \frac{c(n)}{\ell(Q)^{2}} \int_{4 Q}\left|f_{Q, w}^{\varepsilon}(x)-\Phi_{Q} \cdot w^{*}\right|^{2} d x+\frac{c(n)}{\ell(Q)^{2}} \int_{4 Q}\left|\Phi_{Q} \cdot w^{*}\right|^{2} d x+ \\
& c(n) \int_{4 Q}\left|\nabla\left(f_{Q, w}^{\varepsilon}-\Phi_{Q} \cdot w^{*}\right)(x)\right|^{2} d x+c(n) \int_{4 Q}\left|\nabla\left(\Phi_{Q} \cdot w^{*}\right)(x)\right|^{2} d x \\
& \leq \frac{C \varepsilon \ell(Q)^{2}|Q|}{\ell(Q)^{2}}+\frac{c(n)}{\ell(Q)^{2}} \int_{4 Q}\left|\Phi_{Q}\right|^{2}\left|w^{*}\right|^{2} d x+ \\
& C|Q|+c(n) \int_{4 Q}\left|w^{*}\right|^{2} d x \\
& \leq C|Q|+\frac{c(n)}{\ell(Q)^{2}} \int_{4 Q}\left|x-x_{Q}\right|^{2} d x+c(n)|4 Q| \\
& \leq C|Q|+\frac{c(n)}{\ell(Q)^{2}} \sup _{x \in 4 Q}\left|x-x_{Q}\right|^{2} \int_{4 Q} d x \\
& \leq C|Q|+\frac{c(n) 4 \ell\left(Q^{2}\right)}{\ell(Q)^{2}} \int_{4 Q} d x \leq C|Q|+4|Q|=C|Q| .
\end{aligned}
$$

Onde aplicamos as desigualdades (5.2) e (5.3) facilmente, já que $4 Q \subset 5 Q$. 
A continuação, escrevemos:

$$
\begin{aligned}
\theta_{t} \nabla(\mathcal{X} f) & =\left(I+t^{2} L\right)^{-1} t L(\mathcal{X} f) \\
& =\left(I+t^{2} L\right)^{-1} t(-\operatorname{div} A \nabla(\mathcal{X} f)) \\
& =\left(I+t^{2} L\right)^{-1} t(-\operatorname{div}(A f \cdot \nabla \mathcal{X})-\operatorname{div}(A \mathcal{X} \cdot \nabla f)) \\
& =\left(I+t^{2} L\right)^{-1} t(-\operatorname{div}(A f \cdot \nabla \mathcal{X})-A \nabla f \cdot \nabla \mathcal{X}-\mathcal{X} \operatorname{div} A \nabla f) \\
& =\left(I+t^{2} L\right)^{-1} t(\mathcal{X} L f)-\left(I+t^{2} L\right)^{-1} t(A \nabla f \cdot \nabla \mathcal{X})-\left(I+t^{2} L\right)^{-1} t \operatorname{div}(A f \nabla \mathcal{X})
\end{aligned}
$$

Por tanto, segue que:

$$
\begin{aligned}
& \int_{Q} \int_{0}^{\ell(Q)}\left|\theta_{t} \nabla\left(\mathcal{X} f_{Q, w}^{\varepsilon}\right)\right|^{2} \frac{d x d t}{t} \leq \int_{Q}^{\ell(Q)} \int_{0}^{(Q)}\left|\left(I+t^{2} L\right)^{-1} t\left(\mathcal{X} L f_{Q, w}^{\varepsilon}\right)\right|^{2} \frac{d x d t}{t}+ \\
& \int_{Q}^{\ell(Q)} \int_{0}^{\ell}\left|\left(I+t^{2} L\right)^{-1} t \operatorname{div}\left(A f_{Q, w}^{\varepsilon} \nabla \mathcal{X}\right)\right|^{2} \frac{d x d t}{t}+ \\
& \int_{Q}^{\ell} \int_{0}^{\ell(Q)}\left|\left(I+t^{2} L\right)^{-1} t\left(A \nabla f_{Q, w}^{\varepsilon} \cdot \nabla \mathcal{X}\right)\right|^{2} \frac{d x d t}{t} \\
& =I+I I+I I I \text {. }
\end{aligned}
$$

Agora, vamos estudar cada termo separado e estimá-los por uma constante que multiplica $|Q|$.

Para $I$ :

Como $f_{Q, w}^{\varepsilon}=\left(1+(\varepsilon \ell(Q))^{2} L\right)^{-1}\left(\Phi_{Q} \cdot w^{*}\right)$, podemos aplicar $1+(\varepsilon \ell(Q))^{2} L$ a ambos termos da identidade para obter:

$$
\begin{aligned}
\left(1+(\varepsilon \ell(Q))^{2} L\right) f_{Q, w}^{\varepsilon}=\Phi_{Q} \cdot w^{*} & \left.\Rightarrow(\varepsilon \ell(Q))^{2} L\right) f_{Q, w}^{\varepsilon}=\Phi_{Q} \cdot w^{*}-f_{Q, w}^{\varepsilon} \\
& \Rightarrow L f_{Q, w}^{\varepsilon}=(\varepsilon \ell(Q))^{-2}\left(\Phi_{Q} \cdot w^{*}-f_{Q, w}^{\varepsilon}\right)
\end{aligned}
$$


Então $I$ é estimado por:

$$
\begin{aligned}
I & \leq \int_{0}^{\ell(Q)} \int_{Q}\left|\left(I+t^{2} L\right)^{-1} t\left(\mathcal{X}(\varepsilon \ell(Q))^{-2}\left(\Phi_{Q} \cdot w^{*}-f_{Q, w}^{\varepsilon}\right)\right)(x)\right|^{2} \frac{d x d t}{t} \\
& \leq \int_{0}^{\ell(Q)} \int_{\mathbb{R}^{n}}\left|\left(I+t^{2} L\right)^{-1} t\left(\mathcal{X}(\varepsilon \ell(Q))^{-2}\left(\Phi_{Q} \cdot w^{*}-f_{Q, w}^{\varepsilon}\right)\right)(x)\right|^{2} \frac{d x d t}{t} \\
& \leq \int_{0}^{\ell(Q)} \int_{\mathbb{R}^{n}}\left|t\left(\mathcal{X}(\varepsilon \ell(Q))^{-2}\left(\Phi_{Q} \cdot w^{*}-f_{Q, w}^{\varepsilon}\right)\right)(x)\right|^{2} \frac{d x d t}{t} \\
& \leq(\varepsilon \ell(Q))^{-4} \int_{0}^{\ell(Q)} \int_{4 Q}\left|t\left(\left(\Phi_{Q} \cdot w^{*}-f_{Q, w}^{\varepsilon}\right)\right)(x)\right|^{2} \frac{d x d t}{t} \\
& \leq(\varepsilon \ell(Q))^{-4} \int_{0}^{\ell(Q)} t^{2} \int_{5 Q}\left|f_{Q, w}^{\varepsilon}(x)-\Phi_{Q} \cdot w^{*}(x)\right|^{2} \frac{d x d t}{t} \\
& \leq \varepsilon^{-4} \ell(Q)^{-4} \int_{0}^{\ell(Q)} t^{2} C \varepsilon^{2} \ell(Q)^{2}|Q| \frac{d t}{t}=C \varepsilon^{-2} \ell(Q)^{-2}|Q| \int_{0}^{\ell(Q)} t^{2} \frac{d t}{t} \\
& =C \varepsilon^{-2} \ell(Q)^{-2}|Q| \frac{\ell(Q)^{2}}{2} \\
& =C \varepsilon^{-2}|Q| \\
& =C|Q| .
\end{aligned}
$$

Onde $C=C(\lambda, \Lambda, n, \varepsilon)$.

Para $I I$ :

Afirmamos facilmente que $\operatorname{supp}(\nabla \mathcal{X}) \subset 4 Q \backslash 2 Q$. Isso segue pelo fato de que $\mathcal{X}$ é uma função suave que têm valor $1 \mathrm{em} 2 Q$. Usando a terceira desigualdade do lema $(2.1)$ e (5.2) novamente; tem-se que, para $F=Q$ e $E=4 Q \backslash 2 Q$, com $F \cap E=\emptyset$ e $\operatorname{dist}(E, F)=\ell(Q)>0$ :

$$
\begin{aligned}
\int_{Q}\left|\left(I+t^{2} L\right)^{-1} t \operatorname{div}\left(A f_{Q, w}^{\varepsilon} \nabla \mathcal{X}\right)\right|^{2} d x & \leq C e^{-\frac{\ell(Q)}{c t}} \int_{4 Q \backslash 2 Q}\left|A f_{Q, w}^{\varepsilon} \cdot \nabla \mathcal{X}\right|^{2} d x \\
& \leq C e^{-\frac{\ell(Q)}{c t}} \int_{4 Q \backslash 2 Q}\left|f_{Q, w}^{\varepsilon}\right|^{2}|\nabla \mathcal{X}|^{2} d x \\
& \leq C e^{-\frac{\ell(Q)}{c t}}\|\nabla \mathcal{X}\|_{\infty}^{2} \int_{4 Q \backslash 2 Q}\left|f_{Q, w}^{\varepsilon}\right|^{2} d x
\end{aligned}
$$


Aqui, usamos novamente o fato de que $(4 Q \backslash 2 Q) \subset 5 Q$, que $\|\nabla \mathcal{X}\|_{\infty}^{2} \leq \frac{c(n)}{\ell(Q)^{2}}$ e que a integral obtida na última desigualdade já foi estimada, no desenvolvimento de $\left\|\nabla \mathcal{X} f_{Q, w}^{\varepsilon}\right\|_{L^{2}}^{2}$, por $C|Q| \ell(Q)^{2}$ (onde foi usado (5.3) e o fato de que $\left|\Phi_{Q}\right|^{2}$ é limitado por $\left.4 \ell(Q)^{2}\right)$. Logo:

$$
\int_{Q}\left|\left(I+t^{2} L\right)^{-1} t \operatorname{div}\left(A f_{Q, w}^{\varepsilon} \nabla \mathcal{X}\right)\right|^{2} d x \leq C e^{-\frac{\ell(Q)}{c t}}|Q|
$$

e assim, II é limitada como segue:

$$
I I \leq C|Q| \int_{0}^{\ell(Q)} e^{-\frac{\ell(Q)}{c t}} \frac{d t}{t} \leq C|Q| \int_{0}^{\ell(Q)} \frac{c t}{\ell(Q)} \frac{d t}{t} \leq C|Q| \frac{1}{\ell(Q)} \int_{0}^{\ell(Q)} d t=C|Q|
$$

Para $I I I$ :

$$
\begin{aligned}
\int_{0}^{\ell(Q)} \int_{Q}\left|\left(I+t^{2} L\right)^{-1} t\left(A \nabla \mathcal{X} \cdot \nabla f_{Q, w}^{\varepsilon}\right)\right|^{2} \frac{d x d t}{t} & \leq \int_{0}^{\ell(Q)} \int_{\mathbb{R}^{n}}\left|\left(I+t^{2} L\right)^{-1} t\left(A \nabla \mathcal{X} \cdot \nabla f_{Q, w}^{\varepsilon}\right)\right|^{2} \frac{d x d t}{t} \\
& \leq \int_{0}^{\ell(Q)} \int_{\mathbb{R}^{n}}\left|t\left(A \nabla \mathcal{X} \cdot \nabla f_{Q, w}^{\varepsilon}\right)\right|^{2} \frac{d x d t}{t} \\
& \leq C \int_{0}^{\ell(Q)} t \int_{\mathbb{R}^{n}}|\nabla \mathcal{X}|^{2}\left|\nabla f_{Q, w}^{\varepsilon}\right|^{2} d x d t \\
& \leq C\|\nabla \mathcal{X}\|_{\infty}^{2} \int_{0}^{\ell(Q)} t \int_{4 Q}\left|\nabla f_{Q, w}^{\varepsilon}\right|^{2} d x d t \\
& \leq C \frac{c(n)}{\ell(Q)^{2}} \int_{0}^{\ell(Q)} t \int_{4 Q}\left|\nabla f_{Q, w}^{\varepsilon}\right|^{2} d x d t \\
& \leq C \frac{c(n)}{\ell(Q)^{2}} \int_{0}^{\ell(Q)} t C_{1}|Q| d t \\
& \leq C|Q| Q \mid \int_{0}^{\ell(Q)} t \\
& \leq C(Q)^{2} \\
&
\end{aligned}
$$

Onde novamente usamos as limitações efetuadas na estimação de $\|\nabla \mathcal{X}\|_{L^{2}}$. Com $I, I I$ e $I I I$, temos que $I+I I+I I \leq C|Q|$, com $C$ dependendo somente de $\lambda, \Lambda, n$ 
e $\varepsilon$. Junto com o resultado de $\left\|\nabla \mathcal{X} f_{Q, w}^{\varepsilon}\right\|_{L^{2}}^{2}$, segue que

$$
\begin{aligned}
\int_{Q} \int_{0}^{\ell(Q)}\left|\gamma_{t}(x) \cdot\left(S_{t}^{Q} \nabla f_{Q, w}^{\varepsilon}\right)(x)\right|^{2} \frac{d x d t}{t} \leq & C \int_{\mathbb{R}^{n}}\left|\nabla\left(\mathcal{X} f_{Q, w}^{\varepsilon}\right)(x)\right|^{2} d x+ \\
& 4 \int_{Q}^{\ell(Q)} \int_{0}^{\ell}\left|\theta_{t} \nabla\left(\mathcal{X} f_{Q, w}^{\varepsilon}\right)(x)\right|^{2} \frac{d x d t}{t} \\
\leq & C|Q| .
\end{aligned}
$$

Com o qual Lema 5.4 foi provado.

Demonstração do lema 5.3: O argumento principal para a prova do lema 5.3 é o seguinte resultado, cuja prova será atrasada por um momento.

Proposição 5.5 Existe um pequeno $\varepsilon>0$ dependendo somente de $n, \lambda$ e $\Lambda$; e $\eta=\eta(\varepsilon)>0$ tal que para cada vetor unitário $w \in \mathbb{C}^{n}$ e para cada cubo $Q$, pode-se encontrar uma coleção $S_{w}^{\prime}=\left\{Q^{\prime}\right\}$ de sub-cubos diádicos sem sobreposição de $Q$ com as seguintes propriedades:

i) A união dos cubos em $S_{w}^{\prime}$ têm medida não superior a $(1-\eta)|Q|$.

ii) $S e Q^{\prime \prime} \in S_{w}^{\prime \prime}$, a coleção de todos os sub-cubos diádicos de $Q$ não contidos em qualquer/nenhum $Q^{\prime} \in S_{w}^{\prime}$, então:

$$
\frac{1}{\left|Q^{\prime \prime}\right|} \int_{Q^{\prime \prime}} \operatorname{Re}\left(\nabla f_{Q, w}^{\varepsilon}(y) \cdot w\right) d y \geq \frac{3}{4}
$$

$e$

$$
\frac{1}{\left|Q^{\prime \prime}\right|} \int_{Q^{\prime \prime}}\left|\nabla f_{Q, w}^{\varepsilon}(y)\right|^{2} d y \leq(4 \varepsilon)^{-2}
$$

Mais um resultado de natureza puramente geométrica é também necessário.

Lema 5.6 Seja $w$ um vetor unitário em um espaço de $\mathbb{C}^{n}, u, v$ vetores em $\mathbb{C}^{n}$ e $0<\varepsilon \leq 1$ tal que:

(i) $\left|u-\left(u \cdot w^{*}\right) w\right| \leq \varepsilon\left|u \cdot w^{*}\right|$

(ii) $\operatorname{Re}(v \cdot w) \geq \frac{3}{4}$

(iii) $|v| \leq(4 \varepsilon)^{-1}$

Então $|u| \leq 4|u \cdot v|$.

Prova: Primeiramente, deduze-se de (ii) que:

$$
\begin{aligned}
& \frac{3}{4} \leq \operatorname{Re}(u \cdot w) \leq|(v \cdot w)| \\
\Rightarrow & \frac{3}{4}\left|u \cdot w^{*}\right| \leq\left|u \cdot w^{*}\right||(v \cdot w)|=\left|\left(u \cdot w^{*}\right)(v \cdot w)\right| .
\end{aligned}
$$


Mais ainda, de (i) e a desigualdade triangular, temos que:

$$
\begin{aligned}
& |u|-\left|\left(u \cdot w^{*}\right) w\right| \leq\left|u-\left(u \cdot w^{*}\right) w\right| \leq \varepsilon\left|u \cdot w^{*}\right| \\
\Rightarrow & |u| \leq \varepsilon\left|u \cdot w^{*}\right|+\left|\left(u \cdot w^{*}\right)\right||w|=\varepsilon\left|u \cdot w^{*}\right|+\left|u \cdot w^{*}\right|=(1+\varepsilon)\left|u \cdot w^{*}\right| \\
\Rightarrow & 2 u|\leq| u \cdot w^{*} \mid .
\end{aligned}
$$

como ultima observação, veja-se que por (i) e (iii), a seguinte desigualdade é válida:

$$
\begin{aligned}
\left|u-\left(\left(u \cdot w^{*}\right) w\right) \cdot v\right| & \leq\left|u-\left(\left(u \cdot w^{*}\right) w\right)\right||v| \\
& \stackrel{(i)}{\leq} \varepsilon\left|u \cdot w^{*}\right| \cdot|v| \\
& \stackrel{(i i i)}{\leq} \varepsilon\left|u \cdot w^{*}\right| \frac{1}{4 \varepsilon} \\
& =\frac{1}{4}\left|u \cdot w^{*}\right| .
\end{aligned}
$$

Então, para provar o lema 5.10, fazermos uso novamente da desigualdade triangular:

$$
\begin{aligned}
|u \cdot v| & =\left|u \cdot v \pm\left(u \cdot w^{*}\right)(w \cdot v)\right| \\
& =\left|\left(u \cdot w^{*}\right)(w \cdot v)-\left(u \cdot w^{*}\right)(w \cdot v)+u \cdot v\right| \\
& =\left|\left(u \cdot w^{*}\right)(w \cdot v)-\left(\left(u \cdot w^{*}\right) w-u\right) \cdot v\right| \\
& =\left|\left(u \cdot w^{*}\right)(w \cdot v)\right|-\left|\left(\left(u \cdot w^{*}\right) w-u\right) \cdot v\right| \\
& \geq \frac{3}{4}\left|u \cdot w^{*}\right|-\frac{1}{4}\left|u \cdot w^{*}\right| \\
& =\frac{2\left|u \cdot w^{*}\right|}{4} \\
& \geq \frac{1}{4}|u| .
\end{aligned}
$$

$\Rightarrow|u| \leq|u \cdot v|$

Agora vamos provar lema 5.3, assumindo, pelo momento, a Proposição 5.5. Seja $\varepsilon>0$ a ser escolhido mais adiante e cubra-se $\mathbb{C}^{n}$ com un número finito (dependendo de $n$ e $\varepsilon$ ), de cones $\mathcal{C}_{w}$ associados aos vetores unitários win $\mathbb{C}^{n}$ e definido por:

$$
\mathcal{C}_{w}=\left\{u \in \mathbb{C}^{n} /\left|u-\left(u \cdot w^{*}\right) w\right| \leq \varepsilon\left|u \cdot w^{*}\right|\right\}
$$

Como

$$
\mathbb{C}^{n}=\bigcup_{w(n, \varepsilon)} \mathcal{C}_{w}
$$


Segue que

$$
\begin{aligned}
\left|\gamma_{t}(x)\right|^{2} & =\left|\sum_{w(n, \varepsilon)} \mathcal{X}_{\mathcal{C}_{w}}\left(\gamma_{t}(x)\right) \cdot \gamma_{t}(x)\right|^{2} \\
& \leq \sum_{w(n, \varepsilon)}\left|\mathcal{X}_{\mathcal{C}_{w}}\left(\gamma_{t}(x)\right) \cdot \gamma_{t}(x)\right|^{2} \\
& =: \sum_{w(n, \varepsilon)}\left|\mathbf{1}_{\mathcal{C}_{w}}\left(\gamma_{t}(x)\right) \cdot \gamma_{t}(x)\right|^{2} \\
& =: \sum_{w(n, \varepsilon)}\left|\gamma_{t, w}(x)\right|^{2}
\end{aligned}
$$

onde $\mathbf{1}_{\mathcal{C}_{w}}$ denota a função indicadora de $\mathcal{C}_{w}$. Então, basta argumentar que para cada $w$ fixado, devemos dotar uma estimativa de medida Carleson para $\gamma_{t, w}(x)$. Para isso, defina-se:

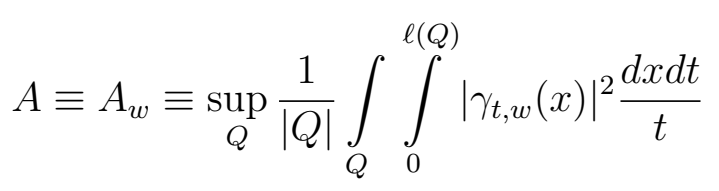

onde o supremo é tomado sobre todos os cubos $Q$. Trucando $\gamma_{t, w}(x)$ por $t$ pequeno e $t$ grande nôs podemos considerar que esta quantidade é quantitativamente finita. Uma vez que um limite a priori independente do truncamento é obtido, podemos passar o limite a través da convergência monótona de Lebesgue. A fim de não introduzir notação adicional ignoramos esse passo simples e podemos assumir que $A<+\infty$.

Agora, fixa-se um cubo $Q$ e seja $Q^{\prime \prime} \in S_{w}^{\prime \prime}$ definido como na proposição 5.7. Considere

$$
v=\frac{1}{\left|Q^{\prime \prime}\right|} \int_{Q^{\prime \prime}} \nabla f_{Q, w}^{\varepsilon}(y) d y \in \mathbb{C}^{n}
$$

Se $x \in Q^{\prime \prime}$ e $\frac{1}{2} \ell\left(Q^{\prime \prime}\right)<t \leq \ell\left(Q^{\prime \prime}\right)$, então:

$$
v=\left(S_{t}^{Q} \nabla f_{Q, w}^{\varepsilon}\right)(x) .
$$

A continuação, de (5.5) da proposição 5.5, vemos que :

$$
\operatorname{Re}(v \cdot w)=\operatorname{Re}\left(\frac{1}{\left|Q^{\prime \prime}\right|} \int_{Q^{\prime \prime}} \nabla f_{Q, w}^{\varepsilon}(y) d y \cdot w\right)=\operatorname{Re}\left(\frac{1}{\left|Q^{\prime \prime}\right|} \int_{Q^{\prime \prime}} \nabla f_{Q, w}^{\varepsilon}(y) \cdot w d y\right) \geq \frac{3}{4}
$$

e

$$
|v| \leq \operatorname{Re} \frac{1}{\left|Q^{\prime \prime}\right|} \int_{Q^{\prime \prime}}\left|\nabla f_{Q, w}^{\varepsilon}(y)\right|^{2} d y \leq(4 \varepsilon)^{-2} \leq(4 \varepsilon)^{-1}
$$


As quais são as condições (ii) e (iii) do teorema 5.10. Tomando-se $u=\gamma_{t, w}(x)$ e a definição de $\gamma_{t, w}$, vemos que, como satisfaz condição (i) do lema 5.10, a seguinte desigualdade é valida:

$$
\begin{aligned}
|u|=\left|\gamma_{t, w}(x)\right| & \leq 4\left|\gamma_{t, w}(x) \cdot v\right| \\
& =4\left|\gamma_{t, w}(x) \cdot\left(S_{t}^{Q} \nabla f_{Q, w}^{\varepsilon}\right)(x)\right| \\
& \leq 4\left|\gamma_{t}(x) \cdot\left(S_{t}^{Q} \nabla f_{Q, w}^{\varepsilon}\right)(x)\right|
\end{aligned}
$$

Observação: Note que a caixa de tipo Carleson $Q \times(0, \ell(Q)]$ pode ser particionada em sub-caixas de tipo Carleson $\left.Q^{\prime} \times\left(0, \ell\left(Q^{\prime}\right)\right)\right]$, com $Q^{\prime}$ descrevendo $S_{w}^{\prime}$, e os retângulos $Q^{\prime \prime} \times\left(\frac{1}{2} \ell\left(Q^{\prime \prime}\right), \ell\left(Q^{\prime \prime}\right)\right]$, para $Q^{\prime \prime}$ descrevendo $S_{w}^{\prime \prime}$. Por tanto:

$\int_{Q} \int_{0}^{\ell(Q)}\left|\gamma_{t, w}(x)\right|^{2} \frac{d x d t}{t}=\sum_{Q^{\prime} \in S_{w}^{\prime}} \int_{Q^{\prime}} \int_{0}^{\ell\left(Q^{\prime}\right)}\left|\gamma_{t, w}(x)\right|^{2} \frac{d x d t}{t}+\sum_{Q^{\prime \prime} \in S_{w}^{\prime \prime}} \int_{Q^{\prime \prime}} \int_{\frac{1}{2} \ell\left(Q^{\prime \prime}\right)}^{\ell\left(Q^{\prime \prime}\right)}\left|\gamma_{t, w}(x)\right|^{2} \frac{d x d t}{t}$

Veja no primeiro termo do lado direito que, que:

$$
\begin{aligned}
\sum_{Q^{\prime} \in S_{w}^{\prime}} \int_{Q^{\prime}} \int_{0}^{\ell\left(Q^{\prime}\right)}\left|\gamma_{t, w}(x)\right|^{2} \frac{d x d t}{t} & =\sum_{Q^{\prime} \in S_{w}^{\prime}} \int_{Q^{\prime}}^{\ell} \int_{0}^{\ell\left(Q^{\prime}\right)}\left|\gamma_{t, w}(x)\right|^{2} \frac{d x d t}{t} \cdot \frac{\left|Q^{\prime}\right|}{\left|Q^{\prime}\right|} \\
& \leq \sup _{Q^{\prime}} \frac{1}{\left|Q^{\prime}\right|} \int_{Q^{\prime}}^{\ell\left(Q^{\prime}\right)} \int_{0}\left|\gamma_{t, w}(x)\right|^{2} \frac{d x d t}{t} \sum_{Q^{\prime} \in S_{w}^{\prime}}\left|Q^{\prime}\right| \\
& \leq A \sum_{Q^{\prime} \in S_{w}^{\prime}}|Q| \\
& \leq A(1-\eta)|Q| .
\end{aligned}
$$

Para o segundo termo, usa-se o resultado (5.9) para obter

$$
\begin{aligned}
\sum_{Q^{\prime \prime} \in S_{w}^{\prime \prime}} \int_{Q^{\prime \prime}} \int_{\frac{1}{2} \ell\left(Q^{\prime \prime}\right)}^{\ell\left(Q^{\prime \prime}\right)}\left|\gamma_{t, w}(x)\right|^{2} \frac{d x d t}{t} & \leq \sum_{Q^{\prime \prime} \in S_{w}^{\prime \prime}} \int_{Q^{\prime \prime}} \int_{\frac{1}{2} \ell\left(Q^{\prime \prime}\right)}^{\ell\left(Q^{\prime \prime}\right)}\left|4 \gamma_{t, w}(x) \cdot\left(s_{t}^{Q} \nabla f_{Q, w}^{\varepsilon}\right)(x)\right|^{2} \frac{d x d t}{t} \\
& \leq 16 \sum_{Q^{\prime \prime} \in S_{w}^{\prime \prime}} \int_{Q^{\prime \prime}} \int_{\frac{1}{2} \ell\left(Q^{\prime \prime}\right)}^{\ell\left(Q^{\prime \prime}\right)}\left|\gamma_{t, w}(x) \cdot\left(s_{t}^{Q} \nabla f_{Q, w}^{\varepsilon}\right)(x)\right|^{2} \frac{d x d t}{t} \\
& \leq 16 \int_{Q}^{\ell(Q)} \int_{0}^{l\left(\left.\gamma_{t}(x) \cdot\left(s_{t}^{Q} \nabla f_{Q, w}^{\varepsilon}\right)(x)\right|^{2} \frac{d x d t}{t}\right.}
\end{aligned}
$$


Assim, $\int_{Q} \int_{0}^{\ell(Q)}\left|\gamma_{t, w}(x)\right|^{2} \frac{d x d t}{t}$ é limitado por

$$
A(1-\eta)|Q|+16 \int_{Q}^{\ell(Q)} \int_{0}^{\ell}\left|\gamma_{t}(x) \cdot\left(s_{t}^{Q} \nabla f_{Q, w}^{\varepsilon}\right)(x)\right|^{2} \frac{d x d t}{t}
$$

Dividendo por $|Q|$ e tomando supremo sobre todos os cubos $Q$, tem-se :

$$
\begin{gathered}
\sup _{Q} \frac{1}{|Q|} \int_{Q} \int_{0}^{\ell(Q)}\left|\gamma_{t, w}(x)\right|^{2} \frac{d x d t}{t} \leq \sup _{Q} \frac{1}{|Q|} 16 \int_{Q}^{\ell(Q)} \int_{0}^{\ell}\left|\gamma_{t}(x) \cdot\left(s_{t}^{Q} \nabla f_{Q, w}^{\varepsilon}\right)(x)\right|^{2} \frac{d x d t}{t}+ \\
+A(1-\eta) .
\end{gathered}
$$

Note-se que o termo esquerdo é exatamente igual a $A$. Dessa forma, temos que:

$$
\begin{gathered}
\quad \eta A \leq 16 \sup _{Q} \frac{1}{|Q|} \int_{Q} \int_{0}^{\ell(Q)}\left|\gamma_{t}(x) \cdot\left(s_{t}^{Q} \nabla f_{Q, w}^{\varepsilon}\right)(x)\right|^{2} \frac{d x d t}{t} \\
A \leq 16 \eta^{-1} \sup _{Q} \frac{1}{|Q|} \int_{Q}^{\ell(Q)} \int_{0}^{l}\left|\gamma_{t}(x) \cdot\left(s_{t}^{Q} \nabla f_{Q, w}^{\varepsilon}\right)(x)\right|^{2} \frac{d x d t}{t}
\end{gathered}
$$

Substituindo novamente o valor de $A$, segue que:

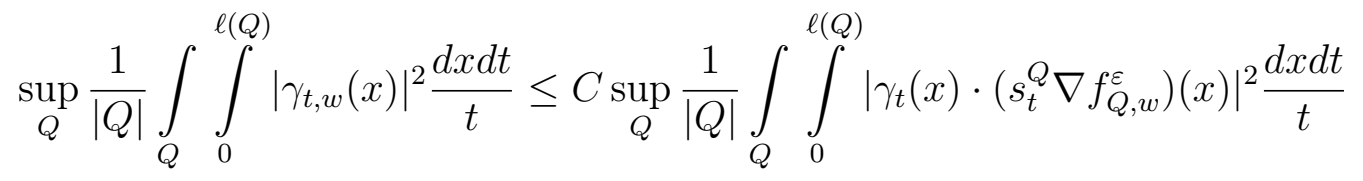

onde $C$ depende de $n, \lambda, \Lambda, \varepsilon$ e $n$. Finalmente, pelo argumento dado depois de (5.11), segue que

$$
\begin{aligned}
\sup _{Q} \frac{1}{|Q|} \int_{Q} \int_{0}^{\ell(Q)}\left|\gamma_{t}(x)\right|^{2} \frac{d x d t}{t} & \leq \sup _{Q} \frac{1}{|Q|} \int_{Q} \int_{0}^{\ell(Q)} \sum_{w \in W}\left|\gamma_{t, w}(x)\right|^{2} \frac{d x d t}{t} \\
& \leq \sum_{w \in W} \sup _{Q} \frac{1}{|Q|} \int_{Q}^{\ell(Q)} \int_{0}^{\ell(Q)}\left|\gamma_{t, w}(x)\right|^{2} \frac{d x d t}{t} \\
& \leq C \sum_{w \in W} \sup _{Q} \frac{1}{|Q|} \int_{Q}^{\ell} \int_{0}^{\ell)}\left|\gamma_{t}(x) \cdot\left(S_{t}^{Q} \nabla f_{Q, w}^{\varepsilon}\right)(x)\right|^{2} \frac{d x d t}{t}
\end{aligned}
$$

Com isso, mostramos a Proposição 5.3, e , a continuação, provaremos o último resultado importante: Proposição 5.5.

Demonstração de Proposição 5.5: Primeiramente, observa-se que:

$$
\nabla\left(\Phi_{Q} \cdot w^{*}\right)(x) \cdot w=\left(w^{*} \cdot w\right)=|w|=1
$$


para um $w \in \mathbb{C}^{n}$ unitário. Logo, segue que:

$$
\begin{aligned}
1-\nabla f_{Q, w}^{\varepsilon}(x) \cdot w & =\nabla\left(\Phi_{Q} \cdot w^{*}\right)(x) \cdot w-\nabla f_{Q, w}^{\varepsilon}(x) \cdot w \\
\rightarrow \quad 1-\nabla f_{Q, w}^{\varepsilon}(x) \cdot w & =\left(\nabla\left(\Phi_{Q} \cdot w^{*}-f_{Q, w}^{\varepsilon}\right)(x)\right) \cdot w=\nabla g(x) \cdot w .
\end{aligned}
$$

onde $g(x)=\Phi_{Q}(x) \cdot w^{*}-f_{Q, w}^{\varepsilon}(x)$. Afirmamos também que:

$$
\left|\int_{Q} 1-\left(\nabla f_{Q, w}^{\varepsilon}(x) \cdot w\right) d x\right| \leq C \varepsilon^{1 / 2}|Q|,
$$

onde $C$ depende somente de $\lambda, \Lambda$ e $n$, e não de $\varepsilon, Q$ e $w$. Para mostrar esta afirmação, fazermos uso de $(5.2),(5,3)$ e da aplicação do seguinte lema a $g$ que será provada como resultado final deste trabalho:

Lema 5.7 Existe uma constante $C=C(n)$ tal que para cada $h \in H^{1}(Q)$ :

$$
\left|\int_{Q} \nabla h d x\right| \leq C \ell(Q)^{\frac{n-1}{2}}\left(\int_{Q}|h|^{2}\right)^{1 / 4}\left(\int_{Q}|\nabla h|^{2} d x\right)^{1 / 4} .
$$

Vamos provar (5.10):

$$
\begin{aligned}
\mid \int_{Q} 1- & \left.\nabla f_{Q, w}^{\varepsilon}(x) \cdot w\right) d x \mid \\
& =\left|\int_{Q} \nabla g(x) \cdot w d x\right| \\
& =\left|\int_{Q} \nabla g(x) d x \cdot w\right| \\
& \leq\left|\int_{Q} \nabla g(x) d x\right| \\
& \leq C \ell(Q)^{\frac{n-1}{2}}\left(\int_{Q}|g|^{2}\right)^{1 / 4}\left(\int_{Q}|\nabla g|^{2} d x\right)^{1 / 4} \\
& =C \ell(Q)^{\frac{n-1}{2}}\left(\int_{Q}\left|\Phi_{Q} \cdot w^{*}-f_{Q, w}^{\varepsilon}\right|^{2}\right)^{1 / 4}\left(\int_{Q}\left|\nabla\left(\Phi_{Q} \cdot w^{*}-f_{Q, w}^{\varepsilon}\right)\right|^{2} d x\right)^{1 / 4} \\
& =C \ell(Q)^{\frac{n-1}{2}}\left(\int_{5 Q}\left|\Phi_{Q} \cdot w^{*}-f_{Q, w}^{\varepsilon}\right|^{2}\right)^{1 / 4}\left(\int_{5 Q}\left|\nabla\left(\Phi_{Q} \cdot w^{*}-f_{Q, w}^{\varepsilon}\right)\right|^{2} d x\right)^{1 / 4} \\
& \leq C \ell(Q)^{\frac{n-1}{2}}\left(\varepsilon^{2} \ell(Q)^{2}|Q|\right)^{1 / 4}|Q|^{1 / 4} \\
& =C \ell(Q)^{\frac{n-1}{2}} \varepsilon^{1 / 2} \ell(Q)^{1 / 2}|Q|^{1 / 4}|Q|^{1 / 4} \\
& =C \ell(Q)^{\frac{n}{2} \varepsilon^{\frac{1}{2}}|Q|^{\frac{1}{2}}=C \varepsilon^{\frac{1}{2}}|Q| \cdot} \\
& \\
& \\
&
\end{aligned}
$$


A última desigualdade é valida pois $|Q|=\ell(Q)^{n} \Rightarrow|Q|^{\frac{1}{2}}=\ell(Q)^{\frac{n}{2}}$. Com isso concluímos que :

$$
\left|\int_{Q} 1-\left(\nabla f_{Q, w}^{\varepsilon}(x) \cdot w\right) d x\right| \leq C \varepsilon^{\frac{1}{2}}|Q|
$$

e a afirmação está provada. Continuando com a demonstração da Proposição 5.5, deduzimos de (5.3), que:

$$
\begin{aligned}
C_{2}|Q| & \geq \int_{5 Q}\left|\nabla\left(f_{Q, w}^{\varepsilon}-\Phi_{Q} \cdot w^{*}\right)\right|^{2} d x \geq \int_{Q}\left|\nabla f_{Q, w}^{\varepsilon}-\nabla \Phi_{Q} \cdot w^{*}\right|^{2} d x \\
& \geq\left.\int_{Q}|| \nabla f_{Q, w}^{\varepsilon}(x)\right|^{2}-2\left[\nabla f_{Q, w}^{\varepsilon}(x) \cdot \nabla\left(\Phi_{Q} \cdot w\right)\right]+\left|\nabla \Phi_{Q} \cdot w\right|^{2} \mid d x \\
& \geq \int_{Q}\left|\nabla f_{Q, w}^{\varepsilon}(x)\right|^{2} d x-2 \int_{Q}\left|\nabla f_{Q, w}^{\varepsilon}(x) \cdot \nabla\left(\Phi_{Q} \cdot w\right)\right| d x+\int_{Q}\left|\nabla \Phi_{Q} \cdot w\right|^{2} d x \\
& \geq \int_{Q}\left|\nabla f_{Q, w}^{\varepsilon}(x)\right|^{2} d x-2 \int_{Q}\left|\nabla f_{Q, w}^{\varepsilon}(x)\right|\left|\nabla\left(\Phi_{Q} \cdot w\right)\right| d x-\int_{Q}\left|\nabla \Phi_{Q} \cdot w\right|^{2} d x
\end{aligned}
$$

Usando o fato de que $2|a b| \leq \frac{1}{2}|a|^{2}+2|b|^{2} \Rightarrow-2|a b| \geq-\frac{1}{2}|a|^{2}-2|b|^{2}$, segue que:

$$
\begin{aligned}
C_{2}|Q| & \geq \int_{Q}\left|\nabla f_{Q, w}^{\varepsilon}(x)\right|^{2} d x-\frac{1}{2} \int_{Q}\left|\nabla f_{Q, w}^{\varepsilon}(x)\right|^{2} d x-2 \int_{Q}\left|\nabla\left(\Phi_{Q} \cdot w\right)\right| d x-\int_{Q}\left|\nabla \Phi_{Q} \cdot w\right|^{2} d x \\
& \geq \frac{1}{2} \int_{Q}\left|\nabla f_{Q, w}^{\varepsilon}(x)\right|^{2} d x-3 \int_{Q}\left|\nabla\left(\Phi_{Q} \cdot w\right)\right| d x \\
& \geq \frac{1}{2} \int_{Q}\left|\nabla f_{Q, w}^{\varepsilon}(x)\right|^{2} d x-3 \int_{Q}\left|\nabla \Phi_{Q}\right||w| d x \\
& =\frac{1}{2} \int_{Q}\left|\nabla f_{Q, w}^{\varepsilon}(x)\right|^{2} d x-3|Q|
\end{aligned}
$$

Somando $3|Q|$ em ambos termos da desigualdade e multiplicando por $2|Q|$, temos que:

$$
\frac{1}{|Q|} \int_{Q}\left|\nabla f_{Q, w}^{\varepsilon}(x)\right|^{2} d x \leq C_{3}
$$

Com $C_{3}$, independente de $\varepsilon$. De (5.10), deduzimos também qu, para $\varepsilon$ suficiente- 
mente pequeno $\left(C \varepsilon^{1 / 2} \leq \frac{1}{8}\right)$, a seguinte desigualdade é valida:

$$
\begin{aligned}
\frac{1}{8} \geq C \varepsilon^{1 / 2} & \geq \frac{1}{|Q|}\left|\int_{Q} 1-\left(\nabla f_{Q, w}^{\varepsilon}(x) \cdot w\right) d x\right| \\
& \geq \frac{1}{|Q|} \operatorname{Re} \int_{Q} 1 d x-\frac{1}{|Q|} \operatorname{Re} \int_{Q} \nabla f_{Q, w}^{\varepsilon}(x) \cdot w d x \\
& =1-\frac{1}{|Q|} \int_{Q} \operatorname{Re}\left(\nabla f_{Q, w}^{\varepsilon}(x) \cdot w\right) d x \\
\Rightarrow \quad & \frac{1}{|Q|} \int_{Q} \operatorname{Re}\left(\nabla f_{Q, w}^{\varepsilon}(x) \cdot w\right) d x \geq \frac{7}{8} .
\end{aligned}
$$

Aqui fazermos uma decomposição de tempo de parada para selecionar uma coleção $S_{w}^{\prime}$ de sub-cubos diádicos $Q$ que são máximos com a propriedade de que uma das seguintes condições abaixo:

$$
\frac{1}{\left|Q^{\prime}\right|} \int_{Q^{\prime}} \operatorname{Re}\left(\nabla f_{Q, w}^{\varepsilon}(x) \cdot w\right) d x \leq \frac{3}{4}
$$

$\mathrm{Ou}$

$$
\frac{1}{\left|Q^{\prime}\right|} \int_{Q^{\prime}}\left|\nabla f_{Q, w}^{\varepsilon}(x)\right|^{2} d x \geq(4 \varepsilon)^{-2} .
$$

sejam satisfeitas (isto é, subdividimos diadicamente $Q$ e paramos no primeiro momento em que (5.11) ou (5.17) aconteçam). Por construção, obtemos (ii) na declaração da proposição 5.5 (para isso, veja a primeira desigualdade (5.11) ). Resta estabelecer (i). Para este fim, seja $B=\cup_{Q^{\prime} \in S_{w}^{\prime}} Q^{\prime}$.

Por tanto, temos que provar que:

$$
|B| \leq(1-\eta)|Q|
$$

Além disso, considere $B_{1}$ ( respetivamente $B_{2}$ ) que consistem na união desses cubos em $S_{w}^{\prime}$ para os quais (5.11)(respetivamente (5.12)) é valido. Assim, temos que $|B| \leq\left|B_{1}\right|+\left|B_{2}\right|$. Já que os cubos em $S_{w}^{\prime}$ são não sobrepostos, segue de (5.12) que:

$$
\left|B_{2}\right| \leq\left|Q^{\prime}\right| \leq(4 \varepsilon)^{2} \int_{Q^{\prime}}\left|\nabla f_{Q, w}^{\varepsilon}(x)\right|^{2} d x \leq(4 \varepsilon)^{2} \int_{Q}\left|\nabla f_{Q, w}^{\varepsilon}(x)\right|^{2} d x \leq(4 \varepsilon)^{2} C_{3}|Q| .
$$

Por outra parte, considerando sub-cubos $Q^{\prime}$ em $B_{1}$ :

$$
\frac{1}{|Q|^{\prime}} \int_{Q^{\prime}} \operatorname{Re}\left(\nabla f_{Q, w}^{\varepsilon}(x) \cdot w\right) d x-\frac{1}{\left|Q^{\prime}\right|} \int_{Q^{\prime}} d x \leq \frac{3}{4}-1=-\frac{1}{4}
$$


$\Rightarrow$

$$
\frac{1}{|Q|^{\prime}} \int_{Q^{\prime}} 1-\operatorname{Re}\left(\nabla f_{Q, w}^{\varepsilon}(x) \cdot w\right) d x \geq \frac{1}{4} \Longrightarrow 4 \int_{Q^{\prime}} 1-\operatorname{Re}\left(\nabla f_{Q, w}^{\varepsilon}(x) \cdot w\right) d x \geq\left|Q^{\prime}\right|
$$

Denotando por $b(x)=1-\operatorname{Re}\left(\nabla f_{Q, w}^{\varepsilon}(x) \cdot w\right),\left|B_{1}\right|$ é estimado por:

$$
\left|B_{1}\right| \leq \sum_{Q^{\prime} \in S_{w}^{\prime}}\left|Q^{\prime}\right| \leq 4 \sum_{Q^{\prime} \in S_{w}^{\prime}} \int_{Q^{\prime}} 1-\operatorname{Re}\left(\nabla f_{Q, w}^{\varepsilon}(x) \cdot w\right) d x=4 \sum_{Q^{\prime} \in S_{w}^{\prime}} \int_{Q^{\prime}} b(x) d x
$$

onde o somatório é tomado sobre todos os cubos $Q^{\prime}$ que compõem $B_{1}$. Dessa forma

$$
\left|B_{1}\right| \leq 4 \sum_{Q^{\prime} \in S_{w}^{\prime}} \int_{Q^{\prime}} b(x) d x=4 \int_{Q} b(x) d x-4 \int_{Q \backslash B_{1}} b(x) d x
$$

O primeiro termo do lado direito é limitado por:

$4 \int_{Q} b(x) d x \leq 4 \int_{Q} 1-\operatorname{Re}\left(\nabla f_{Q, w}^{\varepsilon}(x) \cdot w\right) d x \leq 4\left|\int_{Q} 1-\left(\nabla f_{Q, w}^{\varepsilon}(x) \cdot w\right) d x\right| \leq C \varepsilon^{1 / 2}|Q|$

O segundo termo é controlado em valor absoluto por:

$$
\begin{aligned}
4\left|\int_{Q \backslash B_{1}} b(x) d x\right| & \leq 4 \int_{Q \backslash B_{1}} d x+4 \int_{Q \backslash B_{1}}\left|\nabla f_{Q, w}^{\varepsilon}(x)\right| d x \\
& \leq 4\left|Q \backslash B_{1}\right|+4 \int_{Q \backslash B_{1}}\left|\nabla f_{Q, w}^{\varepsilon}(x)\right| d x \\
& \leq 4\left|Q \backslash B_{1}\right|+4\left(\int_{Q \backslash B_{1}} 1 d x\right)^{1 / 2}\left(\int_{Q \backslash B_{1}}\left|\nabla f_{Q, w}^{\varepsilon}(x)\right|^{2}\right)^{1 / 2} \\
& \leq 4\left|Q \backslash B_{1}\right|+4\left|Q \backslash B_{1}\right|^{1 / 2}\left(\int\left|\nabla f_{Q, w}^{\varepsilon}(x)\right|^{2}\right)^{1 / 2} \\
& \leq 4\left|Q \backslash B_{1}\right|+4\left|Q \backslash B_{1}\right|^{1 / 2} C|Q|^{1 / 2} \\
& \leq 4\left|Q \backslash B_{1}\right|+2\left|Q \backslash B_{1}\right|^{1 / 2} \cdot\left(2\left(C_{3}|Q|\right)^{1 / 2}\right) \\
& \leq 4\left|Q \backslash B_{1}\right|+\varepsilon^{-\frac{1}{2}}\left|Q \backslash B_{1}\right|+\varepsilon^{\frac{1}{2}}\left(4 C_{3}|Q|\right) \\
& =4|Q|-4\left|B_{1}\right|+\varepsilon^{-\frac{1}{2}}|Q|-\varepsilon^{-\frac{1}{2}}\left|B_{1}\right|+C \varepsilon^{\frac{1}{2}}|Q| .
\end{aligned}
$$

pois $\left|Q \backslash B_{1}\right|=|Q|-\left|B_{1}\right|$. Então, temos que $\left|B_{1}\right|$ é limitado por:

$$
\left|B_{1}\right| \leq C \varepsilon^{1 / 2}|Q|+4|Q|-4\left|B_{1}\right|+\varepsilon^{-\frac{1}{2}}|Q|-\varepsilon^{-\frac{1}{2}}\left|B_{1}\right|
$$

$\Rightarrow$

$$
\left(5+\varepsilon^{-\frac{1}{2}}\right)\left|B_{1}\right| \leq\left(4+C \varepsilon^{\frac{1}{2}}+\varepsilon^{-\frac{1}{2}}\right)|Q|
$$


O qual nos dão $\left|B_{1}\right| \leq\left(4-\varepsilon^{\frac{1}{2}}+o\left(\varepsilon^{\frac{1}{2}}\right)\right)|Q|$, se $\varepsilon$ é suficientemente pequeno. Portanto, $|Q| \leq(1-\eta(\varepsilon))|Q|$, com $\eta(\varepsilon) \sim \varepsilon^{1 / 2}$ para um $\varepsilon$ pequeno. Com isso, provamos a Proposição 5.5 a traves da veracidade do Lema 5.7, que será provado, para fechar este capítulo, a continuação.

Demonstração do lema 5.7: Usando o mesmo argumento do capítulo 2, assuma que $Q$ é o cubo $[-1,1]^{n}$ ( e por tanto $\ell(Q)=1$ ) é o caso geral segue por homogeneidade (veja [12]). Seja $M=\left(\int_{Q}|h|^{2}\right)^{1 / 2}$ e $M^{\prime}=\left(\int_{Q}|\nabla h|^{2}\right)^{1 / 2}$.

Se $M \geq M^{\prime}$, vemos facilmente que:

$$
\begin{aligned}
\left|\int_{Q} \nabla h\right|^{2} \leq \int_{Q}|\nabla h|^{2} \leq & \left(\int_{Q}|\nabla h|^{2}\right)^{1 / 2}\left(\int_{Q}|\nabla h|^{2}\right)^{1 / 2} \leq\left(\int_{Q}|h|^{2}\right)^{1 / 2}\left(\int_{Q}|\nabla h|^{2}\right)^{1 / 2} \\
\Rightarrow & \left|\int_{Q} \nabla h\right| \leq\left(\int_{Q}|h|^{2}\right)^{1 / 4}\left(\int_{Q}|\nabla h|^{2}\right)^{1 / 4} .
\end{aligned}
$$

Considere agora $M<M^{\prime}$. Tome-se $t \in(0,1)$, e uma função "cut-off" (como no Capítulo 4) $\varphi \in C_{0}^{\infty}(Q), \operatorname{com} \varphi(x)=1$, quando $d(x, \partial Q)=\operatorname{dist}(x, \partial Q) \geq$ $t$ (aqui tomamos a distância na norma do supremo em $\mathbb{R}^{n}$ ) e $0 \leq \varphi \leq 1$, com $\|\nabla \varphi\|_{\infty} \leq C / t, C=C(n)$. Então:

$$
\int_{Q} \nabla h=\int_{Q}(1-\varphi) \nabla h+\int_{Q} \varphi \nabla h=\int_{Q}(1-\varphi) \nabla h-\int_{Q} h \nabla \varphi
$$

pois $\varphi \equiv 0$ em $\partial Q$. Usando a desigualdade de Holder, nos dão:

$$
\begin{aligned}
\left|\int_{Q} \nabla h\right| & =\left|\int_{Q}(1-\varphi(x)) \nabla h-\int_{Q} h \nabla \varphi(x)\right| \\
& \leq\left|\int_{Q}(1-\varphi(x)) \nabla h\right|+\left|\int_{Q} h \nabla \varphi(x)\right| \\
& \leq \int_{Q}|1-\varphi(x)||\nabla h|+\int_{Q}|h||\nabla \varphi| \\
& \leq\left(\int_{Q}|1-\varphi(x)|^{2}\right)^{1 / 2}\left(\int_{Q}|\nabla h|^{2}\right)^{1 / 2}+\left(\int_{Q}|\nabla \varphi|^{2}\right)^{1 / 2}\left(\int_{Q}|h|^{2}\right)^{1 / 2}
\end{aligned}
$$

Como $\varphi(x)=1$ em $x \in\{x \in Q: d(x, \partial Q) \geq t\}$, então $\varphi \leq 1$ em seu complemen- 
tar, ou seja, $t Q$. Assim:

$$
\begin{aligned}
\left|\int_{Q} \nabla h\right| & \leq\left(\int_{t Q} d x\right)^{1 / 2}\left(\int_{Q}|\nabla h|^{2}\right)^{1 / 2}+\left(\int_{t Q}|\nabla \varphi|^{2}\right)^{1 / 2}\left(\int_{Q}|h|^{2}\right)^{1 / 2} \\
& \leq\left(t^{n}|Q|\right)^{1 / 2}\left(\int_{Q}|\nabla h|^{2}\right)^{1 / 2}+\left(\|\nabla \varphi\|_{\infty}^{2} \int_{t Q} d x\right)^{1 / 2}\left(\int_{Q}|h|^{2}\right)^{1 / 2} \\
& \leq t^{n / 2} M^{\prime}+\left(\frac{c(n)^{2}}{t^{2}} t^{n}|Q|\right)^{1 / 2} M \\
& \leq t^{n / 2} M^{\prime}+c(n) t^{(n-2) / 2} M \\
& =t^{n / 2} M^{\prime}+c(n) t^{n / 2-1} M
\end{aligned}
$$

Como $0<t<1$, então das propriedades do exponente para números menores do que 1 , segue que $t^{n} \leq t^{(n-1)} \leq \ldots \leq t$. Dessa forma, concluímos que:

$$
\begin{aligned}
\left|\int_{Q} \nabla h\right| & \leq C t^{1 / 2} M^{\prime}+C t^{1 / 2-1} M \\
& =C\left(t^{1 / 2} M^{\prime}+t^{-1 / 2} M\right)
\end{aligned}
$$

Resta tomar $t=M / M^{\prime}<1$ para obter :

$$
\left|\int_{Q} \nabla h\right| \leq C\left[M^{1 / 2}\left(M^{\prime}\right)^{1 / 2}+\left(M^{\prime}\right)^{1 / 2} M^{1 / 2}\right]
$$

Substituindo os valores de $M$ e $M^{\prime}$ na equação (5.13), com $C=c(n)$, o lema está provado e finalizamos a ultima demonstração do capítulo 5 .

\subsection{Conclusão}

Para conseguir estimar o operador raiz quadrada, e assim obter (K), faremos um sumário dos resultados obtidos. Para começar, observe que da ultima estimativa conseguida, mostramos o Lema 5.7 . Com isso, a Proposição 5.5 é provada e adicionando o resultado obtido do Lema 5.6, mostra-se Lema 5.3.

Agora, (4.6) segue dos resultados obtidos em Lema 5.3 e Lema 5.4, os quais já foram provados e descritos ao longo deste Capítulo. Dessa forma, as duas hipóteses assumidas no Lema 4.1. são satisfeitas; e então, a desigualdade (4.4) é válida. Lembrando que (4.4) é uma alternativa a (4.1) usando operadores do tipo $P_{t}$ e $\theta_{t}$, segue que (3.2) é satisfeita, pois essa equação foi reescrita por meio 
do operador limitado $\theta_{t}$, obtendo assim (4.1).

Finalmente, da representação de $\sqrt{L} f$ detalhada no Capítulo 3 , vimos que (K) se reduzia a prova de duas estimativas: (3.1) e (3.2), as quais foram provadas no Capítulo 3 e 5, respetivamente. Concluímos então, depois da análise feita ao longo deste trabalho, que a estimativa principal $(\mathrm{K})$, foi provada .

Logo, pelo teorema de J.L.Lions [27], concluímos que o domínio de $\sqrt{L}$ é $H^{1}\left(\mathbb{R}^{n}\right)$ e que para cada $f \in H^{1}\left(\mathbb{R}^{n}\right)$ :

$$
\|\sqrt{L}\|_{L^{2}} \sim\|\nabla f\|_{L^{2}}
$$

Assim, a conjetura proposta por T.Kato, foi resolvida.

Abaixo descrevemos um esquema explicando a conexão entre todos os resultados:

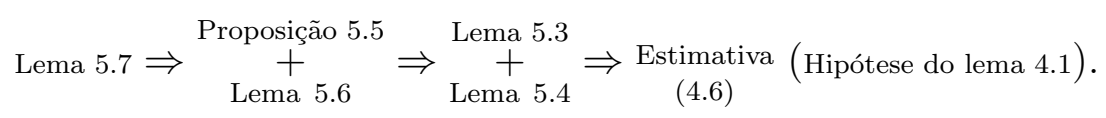

Logo:

$$
\text { Lema } 4.1 \Rightarrow \underset{(4.4)}{\operatorname{Estimativa}} \Rightarrow \underset{(4.1)}{\text { Estimativa }} \Rightarrow \underset{(3.2)}{\text { Estimativa }} \Rightarrow(\mathrm{K})
$$




\section{Apêndice A}

\section{CÁLCULO FUNCIONAL PARA OPERADORES SETORIAIS}

\section{A.1 Funções holomorfas e o espaço $H_{0}^{\infty}$}

Seja $U$ um aberto de $\mathbb{C}$ e $f: U \longrightarrow \mathbb{C}$ uma função. Dizemos que $f$ é diferenciável no ponto $z_{0}$ de $U$ se existe

$$
\lim _{z \rightarrow z_{0}} \frac{f(z)-f\left(z_{0}\right)}{z-z_{0}}
$$

onde o limite é tomado sobre todas as sequências que tendem a $z_{0}$, e para todos elas o quociente têm o mesmo valor $f\left(z_{0}\right)$. Se $f$ é diferenciável e a derivada é contínua em cada ponto $z_{0}$ em $U$, dizemos que $f$ é holomorfa em $U$. Seja $\mathcal{O}(U)$ o conjunto de todas as funções holomorfas sobre um conjunto aberto $U \subset \mathbb{C}$.

Suponha que $A$ denota um operador setorial de ângulo $\omega$ sobre um espaço de Banach $X$. Desejamos achar a existência de operadores da forma:

$$
f(A):=\frac{1}{2 \pi i} \int_{\Gamma} f(z) R(z, A) d z
$$

onde $f \in \mathcal{O}\left(S_{\varphi}\right), \varphi \in(\omega, \pi]$, e o caminho $\Gamma$ 'rodeia' o setor $S_{\omega}$ no sentido positivo. Isto significa em particular que (considerado como uma curva sobre a esfera de Riemann) $\Gamma$ passa a través do $\infty$.

Para dar sentido à anterior integral, a função deve ter um rápido decrescimento no $\infty$. Por isso, introduziremos a noção de limite polinomial.

Dado $\varphi \in(0, \pi]$, considere $f \in \mathcal{M}\left(S_{\varphi}\right) \subset \mathcal{O}\left(S_{\varphi}\right)$, o conjunto de funções holomorfas em $S_{\varphi}$, a menos de um conjunto finito de pontos isolados, chamados polos. Dessa forma 
Definição A.1 $f \in \mathcal{M}\left(S_{\varphi}\right)$ tem limite polinomial $c \in \mathbb{C}$ em 0 , se existe $\alpha>0$ tak que:

$$
f(z)-c=O\left(|z|^{\alpha}\right), \text { quando } z \longrightarrow 0 .
$$

Dizemos que $f$ tem limite polinomial $\infty$ em 0 se $1 / f$ tem limite polinomial 0 em 0 .

A função $f$ tem limite polinomial finito em $0($ em $\infty)$ se existe $c \in \mathbb{C}$ tal que $f$ tem limite polinomial c em $0(\mathrm{em} \infty)$.

Se $f$ tem limite polinomial 0 em $0($ em $\infty)$, dizemos que $f$ é regularmente decadente em $0($ em $\infty)$.

É facil verificar que se $f$ e $g$ têm limites polinomiais em $0($ ou em $\infty$ ) também $f . g$ e $f+g$ os têm.

\section{Observações:}

1. Por "uma função $f$ que tem um limite polinomial em $\infty$ " endenta-se que $f$ têm um limite (dentro de $C_{\infty}$ ) e que esse limite é aproximado ao menos polinomialmente rápido. Note que se esse limite é $\infty$, isso não implica que $f$ é polinomialmente limitado em $\infty$. De fato, considerada como una função sobre $S_{\varphi} \operatorname{com} \varphi \in\left(0, \frac{\pi}{2}\right]$, a função $e^{z}$ tem limite polinomial $\infty$ em $\infty$, porém esta longe de ser polinomialmente limitada

2. Se $f$ é meromórfica em 0 , então $f$ tem limite polinomial em 0 e esse limite é finito se, e somente se, $f$ é holomorfa em 0. Pode-se aplicar o mesmo critério ao ponto $\infty$.

Vejamos novamente a integral de Cauchy anteriormente mencionada. Pela setorialidade de $A$, a função $f$ sendo regularmente decadente em $\infty$ garante a integrabilidade no infinito, pelo menos se $\Gamma$ é eventualmente direito. O mesmo acontece se $f$ é regularmente decadente em $\infty$. Por lo tanto, é natural considerar a chamada classe Dunford-Riesz em $S_{\varphi}$, definida por:

$$
H_{0}^{\infty}\left(S_{\varphi}\right):=\left\{f \in H^{\infty}\left(S_{\varphi}\right) / f \text { é regularmente decadente em } 0 \text { e em } \infty\right\}
$$

onde

$$
H^{\infty}\left(S_{\varphi}\right):=\left\{f \in \mathcal{O}\left(S_{\varphi}\right), f \text { é limitada }\right\}
$$

é uma algebra de Banach de todas as funções holomorfas, limitadas sobre $S_{\varphi}$, munidas com a norma:

$$
\|f\|_{\infty}=\|f\|_{\infty, S_{\varphi}}=\sup \left\{|f(z)|, z \in S_{\varphi}\right\}
$$


Veja facilmente que $H_{0}^{\infty}\left(S_{\varphi}\right)$ é uma álgebra linear na álgebra de $H^{\infty}\left(S_{\varphi}\right)$. Para cada função $f(z)$ em $H_{0}^{\infty}\left(S_{\varphi}\right)$, a função $f(1 / z)$ está também contida em $H_{0}^{\infty}\left(S_{\varphi}\right)$. A descrição seguinte é frequentemente útil.

Lema A.2 Seja $\varphi \in(0, \pi]$, e seja $f: S_{\varphi} \longrightarrow \mathbb{C}$ uma função holomorfa. As seguintes afirmações são equivalentes:

1. A funcção f pertence a $H_{0}^{\infty}\left(S_{\varphi}\right)$.

2. Existe um $c \geq 0$ e um $s>0$ tal que: $|f(z)| \leq C \min \left\{|z|,|z|^{-1}\right\}^{s}$, para todo $z \in S_{\varphi}$

3. Existe um $c \geq 0$ e um $s>0$ tal que: $|f(z)| \leq C \frac{|z|^{s}}{1+|z|^{2 s}}$, para todo $z \in S_{\varphi}$.

4. Existe um $c \geq 0$ e um $s>0$ tal que: $|f(z)| \leq C\left(\frac{|z|}{1+|z|^{2}}\right)^{s}$, para todo $z \in S_{\varphi}$.

Corolário A.3 Dado $\varphi \in(0, \pi]$ função holomorfa $f(z)=\frac{z}{(1+z)^{2}}$ satisfaz a seguinte condição:

$$
\frac{z}{(1+z)^{2}} \leq C \min \left\{|z|,|z|^{-1}\right\}
$$

para todo $z \in S_{\varphi}$.

\section{A.2 Cálculo Funcional Limitado}

Seja A um operador setorial de ângulo $\omega$ sobre um espaço de Banach $X$ e seja $\varphi \in(\omega, \pi]$. Suponha que temos uma sub-álgebra $\mathcal{F} \subset \mathcal{H}^{\infty}\left(\mathcal{S}_{\varphi}\right)$ tal que $f(A)$ está definida pelo cálculo funcional para cada $f \in \mathcal{F}$. Dizemos que o $\mathcal{F}$-cálculo funcional para $A$ é limitado se $f(A) \in \mathcal{B}(X)$ para todo $f \in \mathbb{F}$ e:

$$
\|f(A)\| \leq C\|f\|_{\mathcal{F}}, f \in \mathcal{F}
$$

para alguma constante $C \geq 0$. Aqui, a norma é induzida da norma em $H^{\infty}\left(S_{\varphi}\right)$. Chamamos ao $\inf \left\{C \geq 0,\|f(A)\| \leq C\|f\|_{\mathcal{F}}\right\}$ de cota do $\mathcal{F}$-calculo funcional. 


\section{APÊNDICE A. CÁLCULO FUNCIONAL PARA OPERADORES SETORIAIS}

Se consideramos $\mathcal{F}=H_{0}^{\infty}\left(S_{\varphi}\right)$, temos:

Definição A.4 Dado $f \in \mathcal{F}=H_{0}^{\infty}\left(S_{\varphi}\right)$, tal que $f(A)$ está definida pelo calculo funcional. Dizemos que A têm um $H_{0}^{\infty}$-cálculo funcional limitado,ou simplesmente, que A têm um calculo funcional limitado, se $f(A) \leq \mathcal{B}(X)$, para todo $f \in H_{0}^{\infty}\left(S_{\varphi}\right), e:$

$$
\|f(A)\| \leq\|f\|_{\infty, S_{\varphi}}
$$

Alguns resultados de importante uso ao longo do cálculo funcional limitado serão descritos a continuação:

\section{Subespaços:}

Proposição A.5 Seja $A$ um operador setorial injetivo de ângulo $\omega$ sobre um espaço de Banach $X$. Defina $Y:=\overline{D(A) \cap R(A)}$ e $A_{Y}$ como a parte de $A$ em $Y$ $\left(D\left(A_{Y}\right)=D(A) \cap \overline{D(A) \cap R(A)}\right.$, com $\left.A_{Y} x=A x, x \in D\left(A_{Y}\right)\right)$. Então $A_{Y}$ é um operador setorial de ângulo $\omega$ densamente definido. Mais ainda, se $f \in H_{0}^{\infty}\left(S_{\varphi}\right)$, $\varphi \in(\omega, \pi)$ e $f(A) \in \mathcal{B}(X)$, então $f\left(A_{Y}\right) \in \mathcal{B}(Y)$ e:

$$
\left\|f\left(A_{Y}\right)\right\|_{\mathcal{B}(Y)} \leq\|f(A)\|_{\mathcal{B}(X)} .
$$

Em particular, se A tem cálculo funcional limitado com cota $C$, então $A_{Y}$ também tem cálculo funcional limitado com cota $C$.

\section{Operadores Adjuntos:}

O resultado seguinte, de nenhum modo surpreendente, mostra que a limitação de operadores é preservada quando consideramos o adjunto:

Proposição A.6 Seja A um operador setorial de ângulo $\omega$, densamente definido, $e \varphi \in(\omega, \pi)$, então:

$$
f(A) \in \mathcal{B}(X) \Leftrightarrow f\left(A^{*}\right) \in \mathcal{B}\left(X^{\prime}\right)
$$

para cada $f \in H_{0}^{\infty}\left(S_{\varphi}\right)$, onde $A^{*}$ representa o operador adjunto de $A$. Mais ainda, o operador A tem um cálculo funcional limitado com constante $C$ se, e somente se, $A^{*}$ tem um cálculo funcional limitado com constante $C$.

Lema A.7 Seja A um operador setorial de ângulo $\omega$, com domínio e rango denso. Seja $\varphi \in(\omega . \pi)$, e $C \leq 0$. As seguintes afirmações são equivalentes:

1. $O H_{0}^{\infty}\left(S_{\varphi}\right)$ - cálculo funcional para $A$ é limitado com constante $C$.

2. $O H^{\infty}\left(S_{\varphi}\right)$ - cálculo funcional para $A$ é limitado com constante $C$. 


\section{Apêndice B}

\section{BASE DE RADEMACHER E RESULTADOS AUXILIARES}

\section{B.1 Expansões Binárias}

Defina $S:\{0,1\}^{\mathbb{N}} \rightarrow[0,1]$ por

$$
S(\sigma)=\sum_{k=1}^{\infty} \frac{\sigma_{k}}{2^{k}}, \quad \sigma \in 0,1^{\mathbb{N}} .
$$

Por exemplo, para $\sigma_{1}=0, \sigma_{2}=1, \sigma_{3}=1, \ldots$

$$
S(\sigma)=\frac{0}{2}+\frac{1}{4}+\frac{1}{8}+\cdots=\frac{1}{2}
$$

para $\sigma_{1}=1, \sigma_{2}=0, \sigma_{3}=0, \ldots$

$$
S(\sigma)=\frac{1}{2}+\frac{0}{4}+\frac{0}{8}+\cdots=\frac{1}{2} .
$$

Seja $\sigma \in\{0,1\}^{\mathbb{N}}$. Se existir algum $n \in \mathbb{N}$ tal que $\sigma_{n}=0$ e $\sigma_{k}=1$ para todo $k \geq n+1$, então definindo

$$
\tau_{k}= \begin{cases}\sigma_{k} & : k \leq n-1 \\ 1 & : k=n \\ 0 & : k \geq n+1\end{cases}
$$

obtemos:

$$
S(\sigma)=\sum_{k=1}^{n-1} \frac{\sigma_{k}}{2^{k}}+\sum_{k=n+1}^{\infty} \frac{1}{2^{k}}=\sum_{k=1}^{n-1} \frac{\sigma_{k}}{2^{k}}+\frac{1}{2^{n}}=S(\tau)
$$

Mostra-se que se (i) existir algum $n \in \mathbb{N}$, tal que $\sigma_{n}=0$ e $\sigma_{k}=1$ para todo $k \geq n+1$ ou (ii) se existir algum $n \in \mathbb{N}$ tal que $\sigma_{n}=1$ e $\sigma_{n}=0$ para todo 
$k \geq n+1$, então $S^{-1}(S(\sigma))$ contém exatamente dois elementos, e que de outra forma $S^{-1}(S(\sigma))$ contém somente um elemento.

Defina $\epsilon:[0,1] \rightarrow\{0,1\}^{\mathbb{N}}$, tomando-se $\epsilon(t)$ como sendo o único elemento de $S^{-1}(t)$ se $S^{-1}(t)$ contém exatamente um elemento; e o elemento de $S^{-1}(t)$ que eventualmente é 0 se $S^{-1}(t)$ contém exatamente dois elementos.

Para cada $k \in \mathbb{N}$ definimos $\epsilon_{k}:[0,1] \rightarrow\{0,1\}$ por

$$
\epsilon_{k}(t)=\epsilon(t)_{k}, \quad t \in[0,1]
$$

Então, para cada $t \in[0,1]$,

$$
t=S(\epsilon(t))=\sum_{k=1}^{\infty} \frac{\epsilon_{k}(t)}{2^{k}}
$$

o qual é chamada a expansão binária de $t$.

\section{B.2 Funções de Rademacher}

Para $k \in \mathbb{N}$, a $k$-ésima função de Rademacher $r_{k}:[0,1] \longrightarrow\{-1,+1\}$ é definida por:

$$
r_{k}(t)=1-2 \epsilon_{k}(t), \quad t \in[0,1] .
$$

Então, podemos reescrever a expansão binária de $t \in[0,1]$, em (B.1) como

$$
\sum_{k=1}^{\infty} \frac{r_{k}(t)}{2^{k}}=\sum_{k=1}^{\infty}\left(\frac{1}{2^{k}}-2 \cdot \frac{\epsilon_{k}(t)}{2^{k}}\right)=1-2 \sum_{k=1}^{\infty} \frac{\epsilon_{k}(t)}{2^{k}}=1-2 t .
$$

Assim, defina $r: \mathbb{R} \longrightarrow\{-1,+1\}$ por:

$$
r(x)=(-1)^{[x]},
$$

onde $[x]$ denota o máximo inteiro menor ou igual a $x$. Portanto, para $0 \leq x<1$ temos que $r(x)=1$, para $1 \leq x<2$ tem-se $r(x)=-1$, e $r$ têm período 2 .

Lema B.1 Para qualquer $n \in \mathbb{N}$,

$$
r_{n}(t)=(-1)^{\left[2^{n} t\right]}=r\left(2^{n} t\right), \quad t \in[0,1]
$$

Desta maneira, podemos pensar esta função como uma função que age sobre um conjunto de cubos diádicos de comprimento $2^{n}$. Logo, essa função tem valor constante sobre esses conjuntos diádicos de $[0,1]$, dependendo do valor de $n$. 
Como mais um resultado adicional, tem-se que

Teorema B.2 Se $k_{1}, k_{2}, \ldots, k_{n}$ são inteiros positivos e $k_{1}<k_{2}<\ldots<k_{n}$ então:

$$
\int_{0}^{1} r_{k_{1}}(t) \cdot r_{k_{2}}(t) \cdot \ldots \cdot r_{k_{n}}(t) d t=0 .
$$

Sem perda de generalidade, pode-se considerar uma família de funções $\left\{r_{k}\right\}_{k \in \mathbb{Z}}$ em $L^{2}([0,1])$ com $r_{k}(x)$ tendo valores 1 e -1 , sendo constantes sobre pequenos sub-cubos diádicos do intervalo unitário, dependendo somente de $k$. Por outra parte, do teorema anterior, deduzimos facilmente que:

$$
\int_{0}^{1} r_{k}(t) r_{j}(t) d s=\delta_{k j}
$$

para cada $t \in[0,1]$.

\section{B.3 Estimativas de funções quadráticas}

Lema B.3 Suponha $|\phi(x)| \leq \Phi(x)$ com $\Phi \in L^{1}\left(\mathbb{R}^{n}\right)$ uma função radial. Então para cada $t>0$, a familia de operadores $f \rightarrow \phi_{t} * f$ são uniformemente limitados em $L^{2}\left(\mathbb{R}^{n}\right)$. Mais ainda,

$$
\sup _{t>0}\|\phi(t) * f\|_{\mathcal{B}\left(L^{2}\right)} \leq C(n)\|\Phi\|_{L^{1}} .
$$

Demonstração: Ver [13].

Lema B.4 Considere $\gamma_{t}(x)$ como no Capítulo 4, e suponha que satisfaz

$$
\left\|\gamma_{t}\right\|_{C}=\sup _{Q} \frac{1}{|Q|} \int_{Q}^{\ell(Q)} \int_{0}^{\ell}\left|\gamma_{t}(x)\right| \frac{d t}{t} d x<\infty
$$

onde o supremo é tomado sobre todos os cubos em $\mathbb{R}^{n}$ com lados paralelos aos eixos coordenados. Seja $\rho \in C_{0}^{\infty}\left(\mathbb{R}^{n}\right)$ não negativa, com suporte na bola unitária, $\int \rho d x=1$, e denote $P_{t} f=\rho_{t} * f$, onde $\rho_{t}(x)=t^{-n} p(x / t)$. Então para cada $f \in L^{2}\left(\mathbb{R}^{n}\right)$ :

$$
\int_{0}^{\infty} \int_{\mathbb{R}^{n}}\left|P_{t} f(x)\right|^{2}\left|\gamma_{t}(x)\right|^{2} d x \frac{d t}{t} \leq C\left\|\gamma_{t}\right\|_{C}\|f\|_{L^{2}}^{2}
$$

Demonstração: Ver [13]. 


\section{APENDICE B. BASE DE RADEMACHER E RESULTADOS AUXILIARES}

Proposição B.5 Seja $\psi$ uma função radial de Schwartz com a propriedade de que $\widehat{\psi}(0)=0$ e

$$
\int_{0}^{\infty} \widehat{\psi}_{t}(\xi)^{2} \frac{d t}{t}=1
$$

Seja $Q_{t} f(x)=\psi_{t} * f(x)$. Assuma que que têm-se uma família de operadores $\left\{R_{t}\right\}$ tal que para cada $R_{t}$, estas são individualmente $L^{2}$-limitadas; e para cada $s, t$ maiores que 0, a composição $R_{t} Q_{s}$ verifica:

$$
\left\|R_{t} Q_{s}\right\|_{\mathcal{B}\left(L^{2}\right)} \leq K\left(\min \left\{\frac{t}{s}, \frac{s}{t}\right\}\right)^{\alpha}
$$

para algum $K, \alpha>0$. Então $\left\{R_{t}\right\}$ satisfaz a seguinte estimativa quadrática:

$$
\int_{0}^{\infty} \int_{\mathbb{R}^{n}}\left|R_{t} f(x)\right|^{2} \frac{d t}{t} d x \leq K C(n, \psi, \alpha)\|f\|_{L^{2}}^{2}
$$

Demonstração: Ver Lema 3.2 em [7].

Corolário B.6 Suponha que A é um operador linear limitado em $L^{2}\left(\mathbb{R}^{n}\right)$, com $\|A\|_{\mathcal{B}(X)}$ dependendo somente da dimensão $n$. Então, existe um $0<\theta<1$ tal que:

$$
\|A\|_{\mathcal{B}\left(L^{2}\right)} \leq C(n)\|A\|_{\mathcal{B}\left(L^{2}\right)}^{1-\theta}\|A\|_{\mathcal{B}\left(L^{2}\right)}^{\theta}
$$




\section{Referências Bibliográficas}

[1] P. Auscher, Regularity theorems and heat kernel for elliptics operators, J. London Math. Soc. 54 (1996), 284-296.

[2] P. Auscher, S. Hofmann, J. L. Lewis and Ph. Tchamitchian, Extrapolation of Carleson measures and the analyticity of Kato's square root operator, Acta Math. 187 (2001),161-190.

[3] P. Auscher, S. Hofmann, A. MeIntosh and Ph. Tchamitchian, The Kato square root problem for higher order elliptic operators and systems on $\mathbb{R}^{n}$, J. Evol. Equ. ,1 (2001), 361-385.

[4] P.Auscher, S. Hofmann, C. Muscalu, T. Tao and C. Thiele, Carleson measures, trees, extrapolation, and T(b) theorems, ArXiv, 2002.

[5] P.Auscher, A. Mcintosh And A. Nahmod, Holomorphic functional calculi of operators, quadratic estimates and interpolation, Indiana Univ. Math. J. , 46 (1997), 375-403.

[6] P.Auscher, A. Mcintosh And Ph. Tchamitchian, Heat kernels of second order complex elliptic operators and applications, J. Funct. Anal. 152 (1998), 22-73.

[7] P.Auscher, S. Hofmann, M. Lacey, A. Mcintosh and Ph. TchaMITCHIAN, The solution of the Kato square root problem for second order elliptic operators on $\mathbb{R}^{n}$, Annals of Mathematics. 156 (2002), 633-654.

[8] P. Auscher And J.M. MartelL, Weighted norm inequalities, offdiagonal estimates and elliptic operators. II. Off-diagonal estimates on spaces of homogeneus type, J. Evol. Equ., 7 (2007), no. 2, 265-316.

[9] P. Auscher and Ph. Tchamitchian, Conjecture de Kato sur les ouverts de $\mathbb{R}$, Rev. Mat. Iberoamericana, 8 (1992), 149-199. 
[10] P. Auscher and Ph. Tchamitchian, Square root problem for divergence operators and related topics, Astérisque 249, Soc. Math. France, Paris, 1998.

[11] J. BELL, Rademacher functions, Department of Mathematics, University of Toronto, 2014.

[12] D. Cruz-Uribe And C. Rios, The Kato Problem for operators with weighted ellipticity, Trans. Amer. Math . Soc., 367, no.7 (2015), 4727-4756.

[13] D. Cruz-URIBe And C. Rios, The solution of the Kato square root problem for degenerate elliptic operators with Gaussian bounds, Trans. Amer. Math . Soc., 364, no.7 (2012), 3449-3478.

[14] R. CzAJA, Differential equations with sectorial operators, Wydawnictwo Uniwersytetu Slaskiego, Katowice 2002.

[15] G. David and J.L. Journé, A boundedness criterion for generalized Calderón-Zygmund operators, Annals of Mathematics, 120, (1984), 371-397.

[16] E.B. DAviEs, Heat kernel bounds, conservation of probability and the Feller property, J. Anal. Math, 58 (1992), 99-119.

[17] N. Dunford And B.J. PetTis, Linear operators on summable functions, Trans. Amer. Math. Soc. , 47 (1940), 323-392.

[18] M. Frazier, B. Jawerth And G. Weiss, Littlewood-Paley theory and the study of function spaces, CBMS Regional Conference Series in Mathematics, 79.

[19] L. Grafakos, Classical Fourier Analysis, Second Edition, Springer, 249 (2008).

[20] M. HAASE, The functional calculus for sectorial operators, Operator Theory: Advances and Applications, 169, Basel, 2006.

[21] S. Hofmann, Local Tb Theorems and applications in PDE, Dep. of Mathematics, 2002.

[22] P. Halmos, G. Lumer And J. Schaffer, Square root of operators, University of Chicago and Univerity of Montevideo, 1952. 
[23] S. Hofmann, M. Lacey And A. McIntosh, The solution of the Kato problem for divergence form elliptic operators with Gaussian heat kernel bounds, Annals of Mathematics, 156 (2002), 622-631.

[24] J.L. Jounné, Remarks on Kato's square root problem, Publ. Math , 35 (1991), 299-321.

[25] T. Kato, Fractional powers of dissipative operators, J. Math. Soc. Japan, 13 (1961), 246-274.

[26] H. Komatsu, Fractional powers of Operators, Pac. J. Math. 19 No.2 (1966), 285-346.

[27] J.L. Lions, Espaces d'interpolation et domaines de puissances fractionnaries, J.Math. Soc. Japan , 14 (1962), 233-241.

[28] A. McIntosh, Square root of operators and applications to hyperbolyc PDEs, Proc. Centre Math. Anal. Austral. Natl. Univ. 5,(1984) 124-136.

[29] A. Mc Intosh, Square root elliptic operators, J. Funct. Anal. , 61, (1985), 307-327.

[30] A. Mc Intosh And A. YAgi, Operators of type $\omega$ without a bounded $H^{\infty}$ functional calculus, Microconference on Operators in Analysis, Proc. Centre Math. Anal. Austral. Nat. Univ, 24, (Camberra, 1989), 159-172.

[31] A. Nolasco, Análise Funcional II , Universidade de São Carlos, 2012.

[32] El M. Ouhabaz, Analysis on heat equations on domains, London Mathematical Society Monographs Series, Vol. 31, Princeton University Press, Princeton, NJ, 2005.

[33] S.Semmes, Square function estimates and the T(b) Theorem, Proc. Amer. Math. Soc. , 110 (1990), 721-726.

[34] B. Simon, Real Analysis, A Comprehensive Course in Analysis, Part 1, American Mathematical Society, 2015.

[35] B. Simon, Operator Theory, A Comprehensive Course in Analysis, Part 4, American Mathematical Society, 2015. 
[36] M.WiLson, The Calderón reproducing formula converges unconditionally in $L^{p}$, Dep. of Mathematics, University of Vermont, Burlington, Vermont 05405 . 